\title{
SEMIGRUPOS DINÁMICOS CUÁNTICOS UNIFORMEMENTE CONTINUOS
}

TESIS QUE PRESENTA:

Gabriel López Garza

PARA OBTENER EL GRADO DE MAESTRO EN MATEMÁTICAS

DIRECTOR: DR. ROBERTO QUEZADA BATALLA

UNIVERSIDAD AUTÓNOMA METROPOLITANA-IZTAPALAPA DIVISIÓN DE CIENCIAS BÁSICAS E INGENIERÍA DEPARTAMENTO DE MATEMÁTICAS

JULIO DE 1998 
SEMIGRUPOS DINÁMICOOS

CUÁNTICOS UNIFORMEMENTE

CONTINUOS

\section{TESIS QUE PRESENTA:}

Gabriel López Garza

PARA OBTENER EL GRADO DE MAESTRO EN MATEMÁTICAS 


\section{ÍNDICE}

INTRODUCCIÓN...................................................................

\section{CAPÍTULO I}

Dinámica reducida..............................................................4

Positividad completa............................................................

Semigrupos dinámicos cuánticos.......................................13

Ejemplo 1........................................................................15

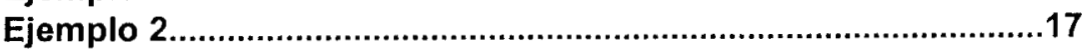

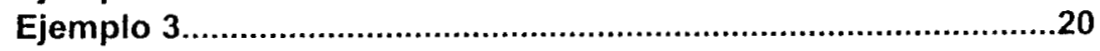

CAPÍTULO II

Teorema de Gorini-Kossakowski-Sudarshan y Lindblad...........22

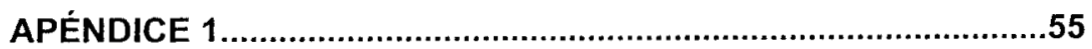

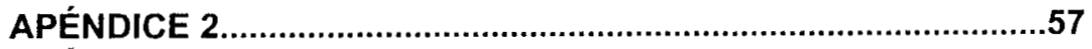

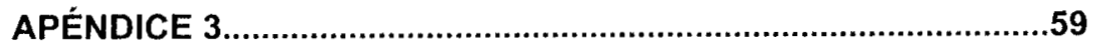

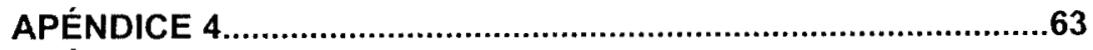

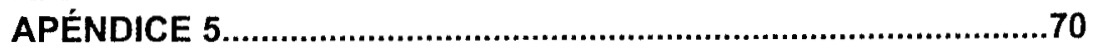

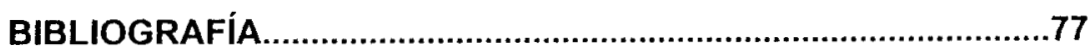




\section{Introducción}

La presente tesis es un trabajo expositorio de algunos aspectos de la teoría de semigrupos dinámicos cuánticos uniformemente continuos, especialmente del teorema de Gorini, Kossakowski, Sudarshan y Lindblad.

En el capítulo I se da una motivación del concepto de semigrupo dinámico cuántico, principalmente de las propiedades de normalidad y de positividad completa, así como algunos ejemplos.

En el capítulo II se da una demostración completa del teorema de Gorini, Kossalowski, Suclarshan y Lindblad que caracteriza a los generadores infinitesimales acotados de semigrupos dinámicos cuánticos uniformemente continuos, estos son operadores acotados del espacio de Banach $\mathcal{B}(\mathcal{H})$ en sí mismo que tienen la siguiente estructura:

$$
\theta(X)=i[H, X]-\frac{1}{2}\left\{L^{*} L X+X L^{*} L-2 L^{*}(X \otimes 1) L\right\}
$$

donde $L: \mathcal{H} \rightarrow \mathcal{H} \otimes \mathcal{K}$, es un operador acotado, $\mathcal{H}$ es un espacio de Hilbert. complejo separable, $\mathcal{K}$ es un espacio de Hilbert y $H$ un operador acotado y autoadjunto en $\mathcal{B}(\mathcal{H})$. Históricamente el teorema fue demostrado primero para espacios de Hilbert de dimensión finita en un trabajo conjunto de Gorini, Kossakowski, Sudarshan $[G-K-S]$ y Lindblad $[L]$ demostró el teorema para un espacio de Hilbert general.

Para demostrar que el generador infinitesemal $\theta$ de un semigrupo dinámico cuántico uniformenente continuo tiene la forma (1) se signió a Parthasarathy $[P a]$ con la diferencia de que en ésta tesis no se usa ningún resultado de probabilidad, sino sólo resultados del anális funcional y del álgebra de operadores, además de haberse desarrollado de una manera exhaustiva los detalles de la demostración que Parthasarathy solamente indica. Para demostrar que todo operador de la forma (1) genera un semigrupodinámico cuántico uniformemente contiuno no se siguió a $[\mathrm{Pa}]$, sino que se utilizó el método emplearlo por 
Chebotarev [Ch1] y García-Quezada $[C-Q$.$] , que ha sido emplearla principal-$ mente con generadores no acotados los cuales no se estudian en el presente trabajo.

Los objetivos principales de la tesis son:

i) Dar una motivación del concepto de semigrupo dinámico cuántico uniformemente continuo y del teorema de Lindblad GKS partiendo de la dinámica reducida de $u$ sistéma cnántico abierto.

ii) Demostrar el teorema de Lindblad sin usar resultados de la teoría de probabilidad, sino sólo aquellos del análisis funcional y del álgebra de operadores.

iii) En lo posible, los resultados previos requeridos, como lo son, el teorema de Gelfand, Naimark, Segal, el teorema de Stone-Von Neumann y demás teoremas adjuntos en los apéndices, están demostrados en la tesis, es clecir, se trató de elaborar un trabajo autocontenido.

Fu conato a las principales limitaciones de este trabajo podemos moncionar que no se pudo complir cabahnente el objetivo iii) ya cque el trabajo se extendió más allá de lo esperado. En particular no inchúmos una demostración del teorema de Kraus mencionado al final del capítulo I ni del teorema que afirma la densidad en sentido débil de los operadores de la forma $U_{s} V_{t}$ con $U_{s}, V_{t}$ grupos uniformemente continuos que cumplen las relaciones de conmutación de Weyl, usada en la Proposición 5 del capítulo II.

De los logros de este traluajo, podemos mencionar que fue posible en la demostración de una parte del teorema de Lindblad combinar la teoría general de semigrupos con el método empleado por Chebotarev, García y Quezada pues usando la serie de potencias para el semigrupo

$$
T_{t}=e^{i t \theta}
$$

que da la teoría general, no es fácil demostrar la positividad completa ni la normalidad de $\left\{T_{l}\right\}$ y usando la couación integral

$$
T_{t}(X)=w_{\ell}^{*} X w_{t}+\int_{0}^{t} d \tau w_{t-\tau}^{*} \Phi\left(T_{\tau}(X)\right) w_{t-\tau}
$$

amque es posible demostrar la positividad completa y la normalidad, no es fácil demostrar que el semigrupo $\left\{T_{t}\right\}$ es uniformente continuo, por ejemplo.

Fu relación con los anteredentes y las posibles extensiones de este trabajo, porlenos decir que, aparte de su relaxión con lísica countica, la teoría de semigrupos dinámicos cuánticos es uno de los caminos por los cuales se busca 
extender los métodos de la probabilidad y de la teoría de procesos estocásticos al caso de variables aleatorias no conmutativas, extendiendo el concepto de semigrupo de Markov al remplazar el espacio de funciones sobre el cual actua este semigrupo por un álgebra de operadores.

La caracterización del generador infinitesimal de un semigrupodinámico cuántico general, es decir, no necesariamonte uniformemente continuo, ha resultado un problema sumamente difícil, sin embargo existen algunos trabajos en esta dirección $[H],[\mathrm{Da} 2],[\mathrm{Br}]$.

Un problema más accesible pero igualmente interesante, es el siguiente: dado un generador formal del tipo Lindblad:

$$
\theta(X)=\Phi(X)-G X-X G^{*}
$$

con coeficientes $\Phi(X)$ y $G$ operadores no acotados, construir un semigrupo dinámico cuántico general con este generador formal (solución de la ecuación maestra). En este caso $\Phi$ es una transformacióncompletamente positiva de $\mathcal{B}(\mathcal{H})$ en un espacio de operadores no acotados. En el capítulo Il de la tesis se prueba que en el caso $\Phi(X)$ y $G$ acotados, La expresión (2) y la (1) son equivalentes. Algunos resultados en esta dirección se encuentran en $[C h 2],[C h 3],[C h-F]$.

El semigrupo construido con este generador formal no necesariamente es conservativo, es decir, no necesariamente preserva la identidad, como lohacen los semigrupos uniformemente continuos y es necesario dar condiciones adicionales para que esto se cumpla (condiciones de conservatividad). Esto motiva otro problema interesante: obtener condiciones necesarias y suficientes o sólo condiciones suficientes para la conservatividad del semigrupo construido.

Este trabajo se realizó con apoyo parcial de CONACYT a través del proyecto Análisis Funcional y Estocástico de Ecuaciones de Evolución clave 0233 P-E9506. 


\section{Capítulo I}

\section{Semigrupos Dinámicos Cuánticos}

Semigrupo dinámico cuántico o semigrupo cuántico, por brevedad, es el nombre comunmente usado para un semigrupo de contracciones, completamente positivas y normales sobre el álgebra de von Neumann $\mathcal{B}(\mathcal{H})$ de un espacio de Hilbert complejo y separable $\mathcal{H}$ que preservan la identidad. En relación con la física cuántica, este concepto permite modelar la evolución de un sistema cuántico abierto, es decir, un sistema que interactúa con el medio que lo rodea. El concepto de semigrupo cuántico también generaliza al de semigrupo de Markov que aparece en la teoría de probabilidad.

En este trabajo estudiaremos a los semigrupos dinámicos cuánticos principalmente en relación con Física, los lectores interesados en su relación con Probabilidad pueden consultar $[M],[E 1],[E 2],[C h 1],[G],[P 1],[A]$.

\section{Dinámica reducida}

La siguiente argumentación formal, nos permitirá motivar la definición de semignupo dinámico cuántico, que se inchuye en la última sección de este capitulo, especialmente las propiedades de normalidad y positividad completa. Considérese, un sistema cuántico abierto $S$, es decir, un sistema cuántico que interacciona con el exterior. En la representación de Schrödinger, para modelar este sistema cuántico se utilizan un espacio de Hilbert complejo y separable $\mathcal{H}_{S}$ con producto interior $\langle\cdot, \cdot\rangle$, conjugarlo lineal en la primera coordenada y lineal en la segunda, y el espacio de Banach $\mathcal{T}\left(\mathcal{H}_{S}\right)$, de los operadores de la clase de traza en $\mathcal{H}_{S}$, con la norma $\|T\|_{1}=\operatorname{tr}\left(T^{*} T\right)^{\frac{1}{2}}$. 
Si $T \in \mathcal{T}$ y además es autoadjunto, tiene una representación de la forma $T=\sum_{n=1}^{\infty} \lambda_{n}\left|\varphi_{n}\right\rangle\left\langle\varphi_{n}\right|$, por el teorema espectral, donde $\left\{\varphi_{n}\right\}_{n=1}^{\infty}$ es un sistema ortonormal completo (sonc) y usamos la notación de Dirac $|\cdot\rangle\langle\cdot|$,para el operador $|\varphi\rangle\langle\psi|$ en $\mathcal{B}\left(\mathcal{H}_{S}\right)$ definido por:

$$
|\varphi\rangle\langle\psi| \eta:=\langle\psi, \eta\rangle \varphi \text { para todo } \eta \in \mathcal{H}
$$

Entonces

$$
\operatorname{tr}(T)=\sum_{n=1}^{\infty} \lambda_{n} \mathrm{y}\|T\|_{1}=\sum_{n=1}^{\infty}\left|\lambda_{n}\right|
$$

pues

$$
\begin{aligned}
\operatorname{tr} T & =\sum_{m=1}^{\infty}\left\langle\varphi_{m}, T \varphi_{m}\right\rangle \\
& =\sum_{m=1}^{\infty}\left\langle\varphi_{m}, \sum_{n=1}^{\infty} \lambda_{n} \mid \varphi_{n}\right\rangle\left\langle\varphi_{n} \mid \varphi_{m}\right\rangle \\
& =\sum_{m=1}^{\infty}\left\langle\varphi_{m}, \sum_{n=1}^{\infty} \lambda_{n}\left\langle\varphi_{n}, \varphi_{m}\right\rangle \varphi_{n}\right\rangle \\
& =\sum_{n, m=1}^{\infty} \lambda_{n} \delta_{m n} \\
& =\sum_{n=1}^{\infty} \lambda_{n}\langle\infty
\end{aligned}
$$

Y de manera similar se obtiene $\|T\|_{1}=\sum_{n=1}^{\infty}\left|\lambda_{n}\right|<\infty$.

Los estados mezclados o matrices de densidad de $S$ son los operadores positivos de traza 1. Estos forman un conjunto convexo $\mathcal{P}\left(\mathcal{H}_{S}\right)$, es decir, si $\rho_{1}, \rho_{2} \in \mathcal{P}\left(\mathcal{H}_{S}\right)$, entonces para $0<\alpha<1, \alpha \rho_{1}+(1-\alpha) \rho_{2}$ es un operador positivo $\mathrm{y}$

$$
\begin{aligned}
\operatorname{tr}\left(\alpha \rho_{1}+(1-\alpha) \rho_{2}\right) & =\sum_{n=1}^{\infty}\left\langle\varphi_{n}, \alpha \rho_{1} \varphi_{n}+(1-\alpha) \rho_{2} \varphi_{n}\right\rangle \\
& =\sum_{n=1}^{\infty}\left\langle\varphi_{n}, \alpha \rho_{1} \varphi_{n}\right\rangle+\sum_{n=1}^{\infty}\left\langle\varphi_{n},(1-\alpha) \rho_{2} \varphi_{n}\right\rangle \\
& =\alpha-(1-\alpha) \\
& =1
\end{aligned}
$$

Llamamos estados puros del sistema a los extremos de $\mathcal{P}\left(\mathcal{H}_{S}\right)$, dados por las proyecciones sobre subespacios de dimensión 1. En la notación de Dirac 
estos estados son de la forma $|\varphi\rangle\langle\varphi|$ con

$$
\begin{aligned}
1 & =\operatorname{tr}(|\varphi\rangle\langle\varphi|)=\sum_{n=1}^{\infty}\left\langle\varphi_{n}, \mid \varphi\right\rangle\left\langle\varphi \mid \varphi_{n}\right\rangle \\
& =\sum_{n=1}^{\infty}\left\langle\varphi_{n},\left\langle\varphi, \varphi_{n}\right\rangle \varphi\right\rangle \\
& =\sum_{n=1}^{\infty}\left\langle\varphi, \varphi_{n}\right\rangle^{2} \\
& =\|\varphi\|^{2} .
\end{aligned}
$$

Como cada $\rho \in \mathcal{P}\left(\mathcal{H}_{S}\right)$ es positivo y acotado, es autoadjunto y, por lo tanto,

$$
\rho=\sum_{n=1}^{\infty} \alpha_{n}\left|\varphi_{n}\right\rangle\left\langle\varphi_{n}\right|
$$

con $\left\{\varphi_{n}\right\}$ un sistema ortonormal completo y

$$
1=\operatorname{tr} \rho=\sum_{k=1}^{\infty} \alpha_{k} .
$$

Los observables acotados del sistema se representan por operarlores antoudjuntos de $\mathcal{B}\left(\mathcal{H}_{S}\right)$ y el valor medio de uno de estos observables $A$ en un estado $\rho$, está dado por $\operatorname{tr}(\rho A)$.

Los estados de $S$ cambian debido a la dinámica interna del sistena y a su interacción con el exterior $R$. El espacio de Hilbert del sisterna complete $(S+R)$ es el producto tensorial $\mathcal{H}_{S} \otimes \mathcal{H}_{R}$ donde $\mathcal{H}_{R}$ es el espacio de Hilbert complejo y separable asociado con el medio exterior $R$. Deseamos describir sólo la dinámica interna del sistema (dinámica reducida), entonces sólo nos interesan los cambios de los estados de $S$.

Supongamos que preparamos al tiempo $t_{0}=0$ un estado inicial del sistema completo $\rho \otimes \omega_{R}$, donde $\rho$ es un estado inicial de $S$, es recir $\rho \in \mathcal{P}\left(\mathcal{H}_{S}\right)$ y $\omega_{R}$ es un estado fijo de referencia de $R$. Denotemos por $U_{t}=e^{-i t H_{i o t}}$ al grupo unitario que describe la evolución reversible del sistema completo, su generador $H_{\text {tot }}$ es un operador autoadjunto en $\mathcal{H}_{S} \otimes \mathcal{H}_{R}$. Entonces, la transformación que describe un cambio de estado en $S$ puede escribirse en la forma

$$
\rho \rightarrow \Lambda_{l} \rho=E\left(U_{l} \rho \otimes \omega_{R} U_{i}^{*}\right)
$$


donde $E$ es el operador (traza parcial o esperanza condicional) de $\mathcal{T}\left(\mathcal{H}_{S} \otimes \mathcal{H}_{R}\right)$ en $\mathcal{T}\left(\mathcal{H}_{S}\right)$ definido mediante la relación

$$
\langle\varphi, E \gamma(\psi)\rangle=\sum_{k=1}^{\infty}\left\langle\varphi \otimes f_{k}, \gamma\left(\psi \otimes f_{k}\right)\right\rangle_{\mathcal{H}_{S} \otimes \mathcal{H}_{n}}
$$

para $\gamma \in \mathcal{T}\left(\mathcal{H}_{S} \otimes \mathcal{H}_{R}\right)$ y $\varphi, \psi \in \mathcal{H}_{S}$, donde $\left\{f_{k}\right\}$ es un sistema ortomormal completo en $\mathcal{H}_{R}$. $E$ está bien definido y $E \gamma \in \mathcal{T}\left(\mathcal{H}_{S}\right)$ pues si $\left\{\varphi_{n}\right\}$ es Im sistema ortonormal completo entonces

$$
\begin{aligned}
\operatorname{tr}(E \gamma) & =\sum_{k}\left\langle\varphi_{k}, E \gamma \varphi_{k}\right\rangle \\
& =\sum_{k, l}\left\langle\varphi_{k} \otimes f_{l}, \gamma \varphi_{k} \otimes f_{l}\right\rangle \\
& =\operatorname{tr} \gamma<\infty .
\end{aligned}
$$

\section{Positividad completa}

La condición de positividad completa, que es más fuerte que la positividad usual $\left(X \geq 0\right.$ implica $\left.\Lambda^{\dagger} X \geq 0\right)$, está motivada por las siguientes condiciones $[K],[L],[D]$. Para cada $t \geq 0$ la transformación $\Lambda_{t}: \mathcal{T}\left(\mathcal{H}_{s}\right) \rightarrow \mathcal{T}\left(\mathcal{H}_{s}\right)$ definida por (1.1)pertenece a una clase especial de transformaciones. Por comodidad, sea $t>0$ fijo y denotemos por $\Lambda$ a $\Lambda_{t}$. Intentemos caracterizar a la clase de transformaciones $\Lambda$ en términos sólo del espacio de Hilbert $\mathcal{H}_{S}$. Podemos suponer que todas las sumas son finitas por simplicidar. Como $\omega_{R}$ es un estado del sistema $R$, es un operador positivo de traza mo, entonces es autoadjunto y tiene una representación de la forma

$$
\omega_{R}=\sum_{v} \lambda_{v}\left|f_{v}\right\rangle\left\langle f_{v}\right|
$$

con $\left\{f_{v}\right\}$ un sonc de $\mathcal{H}_{R}$ y $\left(\lambda_{n}\right)$ una sucesión de números reales. Tomando un sone $\left\{\varphi_{k}\right\}$ en $\mathcal{H}_{s}$ obtenemos la siguiente representación matricial de (I.1)

$$
(\Lambda \rho)_{k, l}=\sum_{\substack{k, n \\ m, n}} \lambda_{\nu} U_{\mu k, \nu m} \rho_{m n n} \bar{U}_{\nu n,, \mu l}
$$

doncle

$$
\left\langle\varphi_{k} \otimes f_{\mu}, U\left(\varphi_{n} \otimes f_{\nu}\right)\right\rangle=U_{k \mu, m \nu}
$$


Entonces la transformación $\Lambda$ puede ser escrita como

$$
\Lambda \rho=\sum_{\alpha} W_{\alpha} \rho W_{c x}^{*}
$$

donde $W_{\text {c }} \in \mathcal{B}\left(\mathcal{H}_{S}\right)$. Para mostrar la equivalencia entre (I.2) y (I.3) tómese $\alpha=(\mu, \nu),\left(W_{\alpha}\right)_{k m}=\sqrt{\lambda_{\nu}} U_{\mu k, \nu m}$, entonces

$$
\begin{aligned}
& (\Lambda \rho)_{k, l}=\left\langle\varphi_{k}, \Lambda \rho \varphi_{l}\right\rangle=\sum_{\mu}\left\langle\varphi_{k} \otimes f_{\mu},\left(U\left(\rho \otimes \omega_{R}\right) U^{*}\right)\left(\varphi_{l} \otimes f_{\mu}\right)\right\rangle_{\mathcal{H}_{S} \otimes \mathcal{H}_{R}} \\
& =\sum_{\mu}\left\langle\varphi_{k} \otimes f_{\mu}, U\left(\sum_{m, \nu}\left|\varphi_{m} \otimes f_{\nu}\right\rangle\left\langle\varphi_{m} \otimes f_{\nu}\right|\right)\left(\rho \otimes \sum_{0} \lambda_{\theta}\left|f_{0}\right\rangle\left\langle f_{0}\right|\right)\right. \\
& \left.\times\left(\sum_{n, \eta}\left|\varphi_{n} \otimes f_{\eta}\right\rangle\left\langle\varphi_{n} \otimes f_{\eta}\right|\right) U^{*}\left(\varphi_{l} \otimes f \mu\right)\right\rangle_{\mathcal{H}_{S} \otimes \mathcal{H}_{\mu}} \\
& =\sum_{\substack{\mu, \nu, \theta, \eta \\
m, n}} \lambda_{0}\left\langle\varphi_{k} \otimes f_{\mu}, U\left(\varphi_{m} \otimes f_{\nu}\right)\right\rangle\left\langle\varphi_{m} \otimes f_{\nu}, \rho \otimes\left(\left|f_{0}\right\rangle\left\langle f_{0}\right|\right)\left(\varphi_{n} \otimes f_{n}\right)\right\rangle \\
& \times\left\langle\varphi_{n} \otimes f_{\eta}, U^{*}\left(\varphi_{l} \otimes f_{\mu}\right)\right\rangle \\
& =\sum_{\substack{\mu, \nu, \theta, \eta \\
m, n}} \lambda_{\theta}\left\langle\varphi_{k} \otimes f_{\mu}, U\left(\varphi_{m} \otimes f_{\nu}\right)\right\rangle\left\langle\varphi_{m} \otimes f_{\nu}, \rho \varphi_{n} \otimes\left\langle f_{\theta}, f_{\eta}\right\rangle f_{\theta}\right\rangle \\
& \times\left\langle\varphi_{n} \otimes f_{\eta}, U^{*}\left(\varphi_{l} \otimes f_{n}\right)\right\rangle \\
& =\sum_{\substack{\mu, \nu, 0, \eta \\
m, n, n}} \lambda_{\theta} U_{k, l, m \nu}\left\langle\varphi_{m}, \rho \varphi_{n}\right\rangle\left\langle f_{\nu},\left\langle f_{\theta}, f_{\eta}\right\rangle f_{\theta}\right\rangle \bar{U}_{n \eta, l / l} \\
& =\sum_{\substack{n, \nu, 0, \eta \\
m, n}} \lambda_{0} U_{k / \mu, m, \nu}\left\langle\varphi_{m}, \rho \varphi_{n}\right\rangle\left\langle f_{\nu}, f_{0}\right\rangle\left\langle f_{\theta}, f_{\eta}\right\rangle \bar{U}_{m, l, l} \\
& =\sum_{\substack{\mu, \nu, 0, \eta \\
m, n}} \lambda_{\theta} U_{k \mu, m \nu}\left\langle\varphi_{m}, \rho \varphi_{n}\right\rangle \delta_{0 \nu} \delta_{\theta \eta} \bar{U}_{n \eta, l \mu} \\
& =\sum_{\substack{\mu, \nu \\
m, n}} \lambda_{\nu} U_{k \mu, m \nu} \rho_{m n} \bar{U}_{n \mu, i \mu}
\end{aligned}
$$

Aquí hemos usado que

$$
\sum_{m, l^{\prime}}\left|\varphi_{m} \otimes f_{\nu}\right\rangle\left\langle\varphi_{m} \otimes f_{\nu}\right|=1_{\mathcal{H}_{S} \otimes \mathcal{H}_{R}}
$$

Para $(\mu, \mu)$ fijo, sea $W_{(\mu, \mu)}$ el operador sobre $\mathcal{H}_{s}$ definido por

$$
\left\langle\varphi_{k}, W_{(\mu, \nu)} \varphi_{m}\right\rangle=U_{k \mu, m \nu}
$$


Entonces, (1.4) puede escribirse en la forma

$$
\begin{gathered}
(\Lambda \rho)_{k, l}=\left\langle\varphi_{k}, \Lambda \rho \varphi_{l}\right\rangle \\
=\sum_{\substack{\mu, \nu \\
m, n}} \lambda_{\nu}\left\langle\varphi_{k}, W_{(\mu, \nu)} \mid \varphi_{m}\right\rangle\left\langle\varphi_{m} \mid \rho \varphi_{n}\right\rangle\left\langle\varphi_{n}, W_{(\mu, \nu)}^{*} \varphi_{l}\right\rangle \\
=\sum_{\substack{\mu, \nu \\
n}} \lambda_{l}\left\langle\varphi_{k}, W_{(\mu, \nu)} \rho \varphi_{n}\right\rangle\left\langle\varphi_{n}, W_{(\mu, \nu)}^{*} \varphi_{l}\right\rangle \\
=\sum_{\mu, \nu} \lambda_{\nu}\left\langle\varphi_{k}, W_{(\mu, \nu)}\left(\rho\left|\varphi_{n}\right\rangle\left\langle\varphi_{n}\right| W_{(\mu, \nu)}^{*} \varphi_{l}\right)\right\rangle \\
=\sum_{\mu, \nu} \lambda_{\nu}\left\langle\varphi_{k}, W_{(\mu, \nu)} \rho W_{(\mu, \nu)}^{*} \varphi_{l}\right\rangle
\end{gathered}
$$

pues $\sum_{m}\left|\varphi_{m}\right\rangle\left\langle\varphi_{m}\right|=1$, por tianto $\Lambda$ tiene la forma (I.3). Podemos mostrar además que $\sum_{\alpha} W_{\alpha} W_{\alpha}^{*}=1$, dado que

$$
\begin{aligned}
& (\Lambda 1)_{k, l}=\sum_{\mu, \nu} \lambda_{\nu}\left\langle\varphi_{k}, W_{(\mu, \nu)} W_{(\mu, \nu)}^{*} \varphi_{l}\right\rangle=\sum_{\mu, \nu} \lambda_{\nu}\left\langle\varphi_{k} \otimes f_{\mu}, U\left(\left(W_{(\mu, \nu)}^{*} \varphi_{l}\right) \otimes f_{\nu}\right)\right\rangle \\
& =\sum_{\mu, \nu} \lambda_{\nu}\left\langle U^{*}\left(\varphi_{k} \otimes f_{\mu}\right),\left(W_{(\mu, \nu)}^{*} \varphi_{l}\right) \otimes f_{\nu}\right\rangle . \\
& =\sum_{\mu, \nu} \lambda_{\nu}\left\langle\widetilde{\varphi_{k}} \otimes \widetilde{f_{\mu l}}\left(W_{(\mu, \nu)}^{*} \varphi_{l}\right) \otimes f_{\nu}\right\rangle \\
& =\sum_{\mu, \nu} \lambda_{\nu}\left\langle\widetilde{\varphi_{k}},\left(W_{(\mu, \nu)}^{*} \varphi_{l}\right)\right\rangle\left\langle\widetilde{f_{\mu}}, f_{\nu}\right\rangle \\
& =\sum_{\mu, \nu} \lambda_{\nu} \overline{\left\langle\varphi_{l},\left(W_{(\mu, \nu)} \widetilde{\varphi_{k}}\right)\right\rangle}\left\langle\widetilde{f_{\mu}}, f_{\nu}\right\rangle \\
& =\sum_{\mu, \nu} \lambda_{\nu}\left\langle\widetilde{\varphi}_{k} \otimes f_{\nu}, U^{*}\left(\varphi_{l} \otimes f_{\mu}\right)\right\rangle\left\langle\widetilde{f_{\mu}}, f_{\nu}\right\rangle \\
& =\sum_{\mu, \nu} \lambda_{\nu}\left\langle\hat{\varphi}_{k} \otimes f_{\nu}, \bar{\varphi}_{l} \otimes \tilde{j}_{\mu}\right\rangle\left\langle\tilde{f}_{\mu}, f_{\nu}\right\rangle \\
& =\sum_{\mu, \nu} \lambda_{\nu}\left\langle\widetilde{\varphi_{k}}, \vec{\varphi}_{l}\right\rangle\left\langle f_{\nu}, \mid \widetilde{f_{\mu}}\right\rangle\left\langle\widetilde{f_{\mu}} \mid f_{\nu^{\prime}}\right\rangle \\
& =\left\langle\widehat{\varphi}_{k}, \bar{\varphi}_{l}\right\rangle \\
& =\left\langle\varphi_{k}, \varphi_{l}\right\rangle
\end{aligned}
$$

donde hemos usado el hecho de que $U^{*}\left(\varphi_{l} \otimes f_{\mu}\right)=\widetilde{\varphi_{l}} \otimes \widetilde{f_{\mu}}$ es un sonc. 
Pascmos aloora a lá representación de Heisenberg. Para cada $A \in \mathcal{B}(\mathcal{H})$, sea $\Lambda^{\dagger} A$ el único elemento en $\mathcal{B}(\mathcal{H})$ tal que

$$
\operatorname{tr}((\Lambda \rho) A)=\operatorname{tr}\left(\rho\left(\Lambda^{\dagger} A\right)\right)
$$

La transformación $\Lambda^{\dagger}: \mathcal{B}\left(\mathcal{H}_{S}\right) \rightarrow \mathcal{B}\left(\mathcal{H}_{S}\right)$ está bien definida si $\Lambda$ es continua, pues para cada $A \in \mathcal{B}(\mathcal{H})$ el funcional $\rho \rightarrow \operatorname{tr}[(\Lambda \rho) A]$ es un funcional lineal continuo en $\mathcal{T}\left(\mathcal{H}_{S}\right)$ y la función $A \rightarrow \operatorname{tr}(\cdot A)$ es un isomorfismo isométrico entre $\mathcal{B}\left(\mathcal{H}_{S}\right)$ y $\mathcal{T}\left(\mathcal{H}_{S}\right)^{*}$, el dual de $\mathcal{T}\left(\mathcal{H}_{S}\right)$. Si la suma es infinita se debe suponer algo en relación con la convergencia de la serie $\sum W_{\alpha} W_{\alpha}^{*}=1$, por remplo, que la convergencia es en sentido fuerte.

Nótese que si $\Lambda$ es de la forma (I.3) obtenemos que $\Lambda^{\dagger}$ tiene la forma

$$
\Lambda^{\dagger} A=\sum_{\alpha} W_{\alpha}^{*} A W_{\alpha}
$$

$W_{c} \in \mathcal{B}\left(\mathcal{H}_{S}\right)$, con $\sum_{\alpha} W_{\alpha}^{*} W_{c \alpha}=1$

En particular, para todo $A \geq 0$,

$$
\begin{aligned}
\left\langle\varphi, \Lambda^{\dagger} A \varphi\right\rangle & =\left\langle\varphi, \sum_{\alpha} W_{\alpha}^{*} A W_{\alpha}\right\rangle \\
& =\sum_{\alpha}\left\langle\varphi, W_{\alpha}^{*} A W_{\alpha} \varphi\right\rangle \\
& =\sum_{\alpha}\left\langle W_{\alpha} \varphi, A W_{\alpha} \varphi\right\rangle \\
& \geq 0
\end{aligned}
$$

entonces $\Lambda^{\dagger}$ preserva positividad, es decir, es una transformación positiva y

$$
\Lambda^{\dagger} 1=\sum_{\alpha} W_{\alpha}^{*} W_{\alpha}=1
$$

Podemos verificar que $\Lambda^{\dagger}$ es una contracción. Si $X$ es acotado

$$
\begin{aligned}
\left|\left\langle\varphi, \Lambda^{\dagger} X \psi\right\rangle\right| & \leq \sum_{\alpha}\left|\left\langle\varphi, W_{\alpha}^{*} X W_{\alpha} \psi\right\rangle\right| \\
& \leq \sum_{\alpha}\|\varphi\|\|\psi\|\|X\|\left\|W_{\alpha}\right\|^{2} \\
& =\|\varphi\|\|\varphi \cdot\|\|X\|
\end{aligned}
$$


pues

$$
\begin{aligned}
\sum_{\alpha}\left\|W_{\alpha}\right\|^{2} & =\sup _{\|\varphi\|=1} \sum_{\alpha}\left\langle W_{\alpha} \varphi, W_{\alpha} \varphi\right\rangle \\
& =\sup _{\|\varphi\|=1}\left\langle\varphi, \sum_{\alpha} W_{\alpha}^{*} W_{\alpha} \varphi\right\rangle \\
& =1
\end{aligned}
$$

de donde

$$
\left\|\Lambda^{\dagger} X\right\| \leq\|X\|
$$

Supóngase ahora que $\left(X_{n}\right)_{n \geq 1}$ es una sucesión de operadores en $\mathcal{B}(\mathcal{H})$ tal que $X_{n} \underset{n}{\rightarrow} X$ en la topología débil* de $\mathcal{B}\left(\mathcal{H}_{S}\right)$, es decir, $\operatorname{tr}\left[X_{n} \rho\right] \underset{n}{\rightarrow} \operatorname{tr}[X \rho]$, para todo $\rho \in \mathcal{T}\left(\mathcal{H}_{S}\right)^{1}$. Tenemos que

$$
\begin{aligned}
\operatorname{tr}\left[\left(\Lambda^{\dagger} X_{n}\right) \rho\right] & =\sum_{m}\left\langle\varphi_{m},\left(\Lambda^{\dagger} X_{n}\right) \rho \varphi_{m}\right\rangle \\
& =\sum_{m}\left\langle\varphi_{m},\left(\sum_{\alpha} W_{\alpha}^{*} X_{n} W_{\alpha}\right) \rho \varphi_{m}\right\rangle \\
& =\sum_{\alpha} \sum_{m}\left\langle\varphi_{m}, W_{\alpha}^{*} X_{n} W_{\alpha} \rho \varphi_{m}\right\rangle \\
& =\sum_{\alpha} \operatorname{tr}\left[W_{\alpha}^{*} X_{n} W_{\alpha} \rho\right] \\
=\sum_{\alpha} \operatorname{tr}\left[X_{n} W_{\alpha}^{*} \rho W_{\alpha}\right] & \sum_{n} \operatorname{tr}\left[X W_{\alpha}^{*} \rho W_{\alpha}\right]=\operatorname{tr}\left[\left(\Lambda^{\dagger} X\right) \rho\right]
\end{aligned}
$$

Entonces, la transformación $\Lambda^{\dagger}$ es normal, es decir, continua en la topología débil* de $\mathcal{B}(\mathcal{H})$. En particular, si $\rho$ es mo operador de rango uno, digamos $\rho=\rho_{\varphi}=|\varphi\rangle\langle\varphi|$ entonces

$$
\left\langle\varphi, \Lambda^{\dagger} X_{n} \varphi\right\rangle=\operatorname{tr}\left[\Lambda^{\dagger} X_{n} \rho_{\varphi}\right] \underset{n}{\rightarrow} \operatorname{tr}\left[\Lambda^{\dagger} X \rho_{\varphi}\right]=\left\langle\varphi, \Lambda^{\dagger} X \varphi\right\rangle
$$

es decir, $\Lambda^{\dagger}$ también es continua en la topología débil de $\mathcal{B}\left(\mathcal{H}_{S}\right)$.

Considérese el espacio de Hilbert n-dimensional $\mathbb{C}^{n}$. Si $\mathcal{H}$ es cualquier espacio de Hilbert entonces $\mathcal{H} \otimes \mathbb{C}^{n}$ puede identificarse con la suma directa $\mathcal{H} \oplus \cdots \oplus \mathcal{H}$ de $n$ sumandos y cualquier operador $X$ en $\mathcal{H} \otimes \mathbb{C}^{n}$ puede escribirse como una matriz $\left(\left(X_{i j}\right)\right)$ donde $X_{i j}$ es un operador en $\mathcal{H}$ para cada

\footnotetext{
'Ver apéndice 5
} 
$1 \leq i, j \leq n$. Si $T: \mathcal{B}\left(\mathcal{H}_{2}\right) \rightarrow \mathcal{B}\left(\mathcal{H}_{1}\right)$ es un operador lineal que satisface las condiciones: (i) $T 1=1$; (ii) $T(X) \geq 0$ si $X \geq 0$; (iii) $T\left(X^{*}\right)=T(X)^{*}$; (iv) $\|T(X)\| \leq\|X\|$; (v) w. $\lim _{n \rightarrow \infty} T\left(X_{n}\right)=T(X)$ si w. $\lim _{n \rightarrow \infty} X_{n}=X$. Definimos el operador lineal $T^{(n)}: \mathcal{B}\left(\mathcal{H}_{2} \otimes \mathbb{C}^{n}\right) \rightarrow \mathcal{B}\left(\mathcal{H}_{1} \otimes \mathbb{C}^{n}\right)$ poniendo $T^{(n)}\left(\left(\left(X_{i j}\right)\right)\right)=\left(\left(T\left(X_{i j}\right)\right)\right), X_{i j} \in \mathcal{B}\left(\mathcal{H}_{2}\right), 1 \leq i, j \leq n$. En tal caso $T^{(n)}(X \otimes B)=T(X) \otimes B$ para todo $X \in \mathcal{B}\left(\mathcal{H}_{2}\right), B \in \mathcal{B}\left(\mathbb{C}^{n}\right)$. No necesariamente $T^{(n)}$ debe preservar positividad. Sin embargo si $T^{(n)}$ preserva la. positividad decimos que $T$ es un operador n-positivo de $\mathcal{B}\left(\mathcal{H}_{2}\right)$ en $\mathcal{B}\left(\mathcal{H}_{1}\right)$. Si $T$ es n-positivo para cala $n=1,2, \ldots$ decimos que $T$ es completamente positivo.

Proposición: Sea $T: \mathcal{B}\left(\mathcal{H}_{2}\right) \rightarrow \mathcal{B}\left(\mathcal{H}_{1}\right)$ un operador lineal completamente positivo. Entonces para cada $X_{1}, \ldots, X_{n} \in \mathcal{B}\left(\mathcal{H}_{2}\right), Y_{1}, \ldots, Y_{n} \in \mathcal{B}\left(\mathcal{H}_{1}\right), n=$ $1,2, \ldots$

$$
\sum_{1 \leq i, j \leq n} Y_{i}^{*} T\left(X_{i}^{*} X_{j}\right) Y_{j} \geq 0
$$

Demostración: Para todo $u \in \mathcal{H}_{1}$ tenemos

$$
\left\langle u, \sum_{i, j} Y_{i}^{*} T\left(X_{i}^{*} X_{j}\right) Y_{j} u\right\rangle=\left\langle\left(\begin{array}{c}
Y_{1} u \\
\vdots \\
Y_{n} u
\end{array}\right),\left(\left(T\left(X_{i}^{*} X_{j}\right)\right)\right)\left(\begin{array}{c}
Y_{1} u \\
\vdots \\
Y_{n} u
\end{array}\right)\right\rangle .
$$

Dado que $\left(\left(X_{i}^{*} X_{j}\right)\right) \geq 0$ en $\mathcal{H}_{2} \otimes \mathbb{C}^{n}$ y por esta razón $\left(\left(T\left(X_{i}^{*} X_{j}\right)\right)\right) \geq 0$ en $\mathcal{H}_{1} \otimes \mathbb{C}^{n}$ de aquí se sigue que el lado derecho de la ecuación de arriba es no negativo.

Un teorema de Kraus $[K]$, afirma que una transformación normal $T$ es completamente positiva si y sólo si, se puede representar en la forma (I.5). Si $\mathcal{H}_{S}$ es separable entonces el índice $\alpha$ se puede tomar en un conjunto contable.

Nuestra argumentación y el teorema de Kraus nos motivan a postular que la dinámica reducida de un sistena cuántico abierto debe ser describa por transformaciones normales completamente positivas. La importancia de la

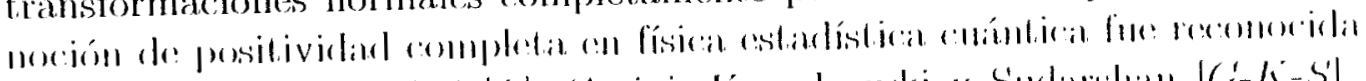

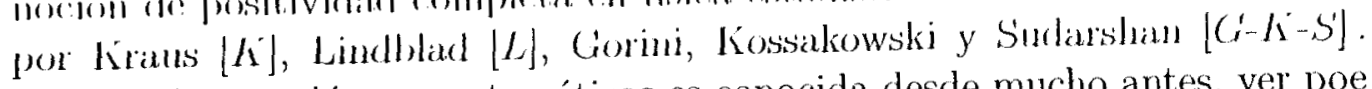
Aunque ésta noción en matemáticas es conocida desde mucho antes, ver poe ejemplo Stinespringe, 1955, Proceedings AMS.

Para describir la evolución temporal de los estarlos de $S$ se necesita ahora dejar que el tiempo $t$ se mueva en $[0, \infty)$, obtenemos de esta manera una 
familia $\left\{T_{\imath}^{\dagger} \mid t \geq 0\right\}$ uniparamétrica de operadores lineales sobre $\mathcal{T}\left(\mathcal{H}_{S}\right)$ o bien, una familia $\left\{T_{t} \mid t \geq 0\right\}$ de operadores lineales sobre $\mathcal{B}\left(\mathcal{H}_{S}\right)$ segín se trabaje en la represcontación de Schrörlinger o en la representarión de Ireisen. berg respectivamente. En general, esta familia no es un semigrupo, pero si suponemos que tiene esta propiedad obtenemos una descripción aproximarla de la covolución de muchos fonomenos físieos.

\section{Semigrupos dinámicos cuánticos}

A lo largo de este trabajo utilizaremos la representación de Heisenberg y consideraremos la siguiente definición de semigrupo dinámico cuántico:

Definición: Un semigrupo dinámico cuántico (s.d.c.) en un espacio de Hilbert complejo y seqarable $\mathcal{H}$ es una familia uniparamétrica $\left\{T_{t} \mid t \geq 0\right\}$ de operadores en el espacio de $\mathcal{B}(\mathcal{H})$ que satisface las siguientes condiciones:

(i) $T_{0}(X)=X$ para todo $X \in \mathcal{B}(\mathcal{H}), T_{t} T_{s}=T_{t+s}$ para todo $s, t \geq 0$;

(ii) $\lim _{t \rightarrow 0}\left\|T_{t}(X)-X\right\|=0$ para todo $X \in \mathcal{B}(\mathcal{H})$;

(iii) $T_{\iota}(1)=1,\left\|T_{t}(X)\right\| \leq\|X\|$,

$$
\text { w. } \lim _{n \rightarrow \infty} T_{l}\left(X_{n}\right)=T_{l}(X) \text { si } w \cdot \lim _{n \rightarrow \infty} X_{n}=X ;
$$

(iv) para todo $X_{i}, Y_{i} \in \mathcal{B}(\mathcal{H}), 1 \leq i \leq n, n=1,2, \ldots$

$$
\sum_{i, j} Y_{i}^{*} T_{l}\left(X_{i}^{*} X_{j}\right) Y_{j} \geq 0
$$

Un semigrupo dinámico cuántico $\left\{T_{L} \mid t \geq 0\right\}$ se dice uniformemente continuo si $\lim _{t \rightarrow 0} \sup _{\|X\| \leq 1}\left\|T_{t}(X)-X\right\|=0$.

Para los semigrupos uniformemente continuos existe, según la teoría gencral de semigrupos, un operador acotarlo $\theta$ en $\mathcal{B}(\mathcal{H})$ tal que

$$
O(X)=\lim _{t \rightarrow 0} \frac{1}{t}\left(T_{t}(X)-X\right) \text { para todo } X \in \mathcal{B}(\mathcal{H})
$$

donde el límite es en la norma de operadores. Tendremos entonces $T_{t}(X)=$ $e^{t \theta}(X)$ para todo $t \geq 0$. Pero la condición (iii) y especialmente (iv) determinan a una clase más restringida de semigrupos, los completamente positivos. Entonces el generador infinitesimal de estos semigrupos debe tener una estructura especial cue corresponda con la condición de positivilad completa. 
La descripción de estos generadores es uno de los objetivos principales de esta tesis.

Continuando con la discusión de la secciómn anterior, consideremos el caso más simple, es decir, cuando $\operatorname{dim} \mathcal{H}=N$. En este caso, $\operatorname{dim} \mathcal{B}(\mathcal{H})=N^{2}$, sea $\left(F_{k}\right)_{k=0}^{N^{2}-1}$ una base de $\mathcal{B}(\mathcal{H})$ con $F_{0}=1$ y escribamos

$$
W_{\alpha}(t)=\sum_{k=0}^{N^{2}-1} \alpha_{k}(t) F_{k}
$$

entonces

$$
W_{\alpha}^{*}(t)=\sum_{k=0}^{N^{2}-1} \overline{\alpha_{k}(t)} F_{k}^{*}
$$

y usando (I.5) con un sólo sumando $(\alpha=1)$ tenemos

$$
\begin{aligned}
\Lambda_{l}^{\dagger} X & =\left(\sum_{k} \overline{\alpha_{k}(t)} F_{k}^{*}\right) X\left(\sum_{k} \alpha_{k}(l) F_{k}\right) \\
& =\left(\sum_{k, l} \overline{\alpha_{k}(l)} \alpha_{l}(l) L_{k}^{\prime *} X F_{k}\right)
\end{aligned}
$$

La matriz $\left(\overline{\alpha_{k}(t)} \alpha_{l}(t)\right)_{k, l=0}^{N^{2}-1}$ es positiva definida y por lo tanto autoadjumla, es decir $\Lambda_{t}^{\dagger} X$ tiene la forma

$$
\sum_{k, l=0}^{N^{2}-1} c_{k, l}(t) F_{k}^{*} X F_{l}
$$

con $\left(c_{k, l}(t)\right)_{k, l=0}^{N^{2}-1}$ una matriz definida positiva. El generador está dado por la expresión:

$$
\begin{aligned}
\theta(X) & =\lim _{\varepsilon \rightarrow 0} \frac{\Lambda_{\varepsilon} X-X}{\varepsilon} \\
& =\lim _{\varepsilon \rightarrow 0} \frac{1}{\varepsilon}\left(\sum_{k, l}^{N^{2}-1} c_{k, l}(\varepsilon) F_{k}^{*} X F_{l}-X\right) \\
& =\lim _{\varepsilon \rightarrow 0}\left\{\frac{c_{00}(\varepsilon)-1}{\varepsilon} X+\sum_{k=1}^{N^{2}-1} \frac{c_{k \cdot 0}(\varepsilon)}{\varepsilon} F_{k}^{*} X+\cdots\right\} \\
& =A^{*} X+X A+\sum_{k, l}^{N^{2}-1} a_{k, l} F_{h}^{*} X F_{l}
\end{aligned}
$$


suponiendo que todos los límites existen, donde $\left(a_{k, l}\right)_{k, l=1}^{N^{2} \ldots 1}$ es una matriz definida positiva y $A=\sum_{k=0}^{N^{2}-1} c_{0 k} F_{k}$. Si además suponemos ${ }^{2}$ que $\theta(1)=0$ obtenemos que

$$
A+A^{*}=-\sum_{k, l=1}^{N^{2}-1} a_{k, l} F_{k}^{*} F_{l}
$$

Entonces,

$$
\theta(X)=\frac{1}{2} \sum_{k, l}^{N^{2}-1} a_{k, l}\left\{F_{k}^{*}\left[X, F_{l}\right]+\left[F_{k}^{*}, X\right] F_{l}\right\}
$$

Si agregamos el término $i[H, X]$ que está asociado con la dinánica interna del sistema, con $H=H^{*}$ y regresamos a la forma de (I.5) tenemos que

$$
\begin{aligned}
\theta(X) & =i[H, X]+\frac{1}{2} \sum_{\alpha}\left\{W_{\alpha}^{*}\left[X, W_{\alpha}\right]+\left[W_{\alpha}^{*}, X\right] W_{\alpha}\right\} \\
& =i[H, X]+\sum_{\alpha} W_{\alpha}^{*} X W_{\alpha}-\frac{1}{2}\left[\sum_{\alpha} W_{\alpha}^{*} W_{r}, X\right]_{+} \\
& =i[H, X]+\Phi(X)-\frac{1}{2}[\Phi(1), X]_{+}
\end{aligned}
$$

con $\Phi(X)=\sum_{\alpha} W_{\alpha}^{*} X W_{\alpha}$. Donde $[\cdot, \cdot]$ denota al conmutador y $[\cdot, \cdot]_{+}$denota al anticonmutador.

Gorini, Kossakowski y Sudarshan $[G-K-S]$ demostraron que ésta es la forma más general del generador de un semigrupo dinámico cuántico 1 miformemente continuo en el caso cuando $\mathcal{H}$ es de dimensión finita. De manera independiente Lindblad $[L]$ demostró este mismo resultado para cualquicr espacio de Hilbert complejo separable $\mathcal{H}$. No existe hasta la fecha una caracterización similar para el generador de un semigrupo dinámico cuántico general, es decir, no necesariamente uniformemente continuo. Pero existen algmos trabajos en esta dirección, por ejemplo, Holevo, On the structure of the generators of covariant QDS, J.Functional Analysis.

El objetivo principal de este trabajo es revisar estos resultados, escribiéndolos de una manera autocontenida.

Para finalizar este capitulo consideremos algunos ejemplos.

Ejemplo 1: Si $H$ es un operador acotado y autoadjunto en un espacio de Ifilbert $\mathcal{H}$, sea $e^{i t / l}$ el grupo mintario continuo en la norma de $\mathcal{B}(\mathcal{H})$ generarlo

\footnotetext{
${ }^{2}$ Ver la l'roposición I dol capsítulo ll
} 
por $i H$. Sea

$$
P_{t}(X)=e^{-i t H} X e^{i t H}
$$

entonces $P_{t}$ es un grupo dinámico cuántico, llamado semigrupo de Heisenberg. Efectivamente,

(i) $P_{0}(X)=X$,

$$
\begin{aligned}
P_{s+t}(X) & =e^{-i(s+t) H} X e^{i(s+t) ! l} \\
& =e^{-i s H}\left(e^{-i t H} X e^{i t H}\right) e^{i s H} \\
& =P_{s} P_{t}(X) \text { para todo } s, t \geq 0 .
\end{aligned}
$$

(ii) Para carla $X \in \mathcal{B}(\mathcal{H})$ tenemos que

$$
\begin{aligned}
\left\|P_{t}(X) \varphi-X \varphi\right\| & =\left\|e^{-i t H} X e^{i t H} \varphi-X \varphi\right\| \\
& \leq\left\|X e^{i t H} \varphi-X \varphi\right\|+\left\|e^{-i t H} X \varphi-X \varphi\right\| \\
& \leq\|X\|\|\varphi\|\left(\left\|e^{i t H}-1\right\|+\left\|e^{-i t H}-1\right\|\right)
\end{aligned}
$$

entonces

$$
\begin{aligned}
\left\|P_{t}(X)-X\right\| & =\sup _{\|\varphi\|=1}\left\|P_{t}(X) \varphi-X \varphi\right\| \\
& \leq\|X\|\left(\left\|e^{i l \|}-1\right\|+\left\|e^{-i t I}-1\right\|\right)
\end{aligned}
$$

la última expresión tiende a cero cuando $t$ tiende a cero, por la continuidad de $e^{i t I I}$ en la norma de $\mathcal{B}(\mathcal{H})$. Además, $\sup _{\|X\| \leq 1}\left\|P_{t}(X)-X\right\| \leq\left\|e^{i t H}-1\right\|+$ $\left\|e^{-i t H}-1\right\|$ que tiende a cero cuando $t \rightarrow 0 \mathrm{y}$ por lo tanto $P_{t}$ es uniformemente continuo

(iii) $P_{\iota}(1) u=e^{-i t / l} 1 e^{i t \prime \prime} u=u$,

$$
\begin{aligned}
\left\|P_{l}(X)\right\| & =\left\|e^{-i t H} X e^{i t u}\right\| \\
& \leq\|X\|
\end{aligned}
$$

Y si w. $\lim _{n \rightarrow \infty} X_{n}=X$ entonces

$$
\left\langle u, X_{n} v\right\rangle \underset{n}{\rightarrow}\langle u, X v\rangle \text { para todo } u, v \in \mathcal{H}
$$

así que

$$
\left\langle e^{i t H} u, X_{n} e^{i t H} v\right\rangle \underset{n}{\rightarrow}\left\langle e^{i t H} u, X e^{i t H} v\right\rangle
$$


$y$, tomando adjuntos obtenemos que

$$
\left\langle u, e^{-i t H} X_{n} e^{i t H} v\right\rangle \underset{n}{\rightarrow}\left\langle u, e^{-i t H} X e^{i t H} v\right\rangle
$$

por tanto $w . \lim _{n \rightarrow \infty} P_{t}\left(X_{n}\right)=P(X)$.

(iv) Sean $X_{i}, Y_{i} \in \mathcal{B}(\mathcal{H}), 1 \leq j, k \leq n, n=1,2, \ldots$ entonces

$$
\begin{aligned}
0 & \leq\left\langle\sum_{j} X_{j} e^{i t H} Y_{j} u, \sum_{j} X_{j} e^{i t H} Y_{j} u\right\rangle \\
& =\left\langle u, \sum_{j, k} Y_{j}^{*} e^{-i t I I} X_{j}^{*} X_{k} e^{i t H} Y_{k}\right\rangle
\end{aligned}
$$

Finalmente, para el generador del semigrupo tenemos

$$
\begin{aligned}
\frac{d}{d t} P_{t}(X) & \left|{ }_{t=0}=\left(-i H e^{-i t H} X e^{i t H}+i e^{-i t H} X H e^{i t H}\right)\right|_{t=0} \\
= & -i(H X-X H) \\
= & -i[H, X]
\end{aligned}
$$

Ejemplo 2: Sea $\{w(t) \mid t \geq 0\}$ un movimiento Browniano con $w(0)=0 \mathrm{y}$ sea $L$ un operador autoadjunto en el espacio de Hilbert complejo y separable $\mathcal{H}=L_{2}\left(\mathbb{R}^{d}\right)$. Definimos

$$
T_{t}(X)=\mathbb{E} e^{i w(\ell) L} X e^{-i w(t) L}
$$

donde $\mathbb{E}$ denota la esperanza con respecto a la medida de probabilidad del proceso $w$ en el sentido de Bochner, es recir, para cada $u \in \mathcal{H}$ la función $\mathcal{H}$-valuada $e^{i w(t) L} X e^{-i w(t) L} u$ es Bochner integrable $e^{3}$. La medibilidad fuerte de la función $w \rightarrow e^{i w(t) L} X e^{-i w(t) L} u$ para cada $t \geq 0$ y $u \in \mathcal{H}$ se sigue de su medibilidad en sentido débil y del teorema 1 de la sección 4 del capítulo $\mathrm{V}$ de $[Y]$, su integrabilidarl es consecuencia del teorema 1 de la sección 5 del mismo libro y la desigualdad

$$
\left\|e^{i w(l) L} X e^{-i w(\ell) L} u\right\| \leq\|X\|\|u\|
$$

para todo $t \geq 0, u \in \mathcal{H}$. Entonces la esperanza está bien definida en el sentido de Bochner.

\footnotetext{
${ }^{3}$ Ver secciones 4 y 5 del capítulo $\mathrm{V}$ de $[Y]$.
} 
Dado que el movimiento Browniano estandard tiene incrementos estatcionariamente independientes tenemos

$$
\begin{aligned}
T_{t+s}(X) & =\mathbb{E} e^{i(w(t+s)-w(s)) L} e^{i w(s) L} X e^{-i w(s) L} e^{-i(w(t+s)-w(s)) L} \\
& =T_{t}\left(T_{s}(X)\right)
\end{aligned}
$$

Si $L$ es acotado se sigue que $\left\{T_{t}\right\}$ es un sde uniformemente continuo. En efecto, si $(a d L)(X)=[L, X]$, tenemos que la sucesión

$$
f_{t}^{n}(w, X)=\sum_{k=0}^{n} \frac{(i w(t))^{k}}{k !}(a d L)^{k}(X)
$$

$n=0,1,2, \ldots$ converge en la norma de $\mathcal{B}(\mathcal{H})$ a $e^{i w(l) L} X e^{-i w(t) L}$ para cada $t$. En particular

$$
f_{t}^{n}(u, X) u \underset{n}{\rightarrow} e^{i w(t) L} X e^{-i w(t) L} u
$$

y además

$$
\begin{aligned}
\left\|f_{t}^{n}(u, X) u\right\| & \leq \sum_{k=0}^{n} 2^{k}\|L\|^{k}\|X\|\|u\| \frac{|w(t)|^{k}}{h !} \\
& \leq e^{2\|L\| \| w(t) \mid}\|X\|\|u\|,
\end{aligned}
$$

pues $\left\|(a d L)^{k}(X)\right\| \leq 2^{k}\|L\|^{k}\|X\|$. Entonces por el teorema de convergencia dominada para integrales de Bodher tenemos que

$$
\begin{aligned}
\mathbb{E}\left(c^{i m(t) l} X_{e}-i w(t) l\right) & =\lim _{n} \mathbb{E}\left(\int_{l}^{n}(w, X)\right) \\
& =\lim _{n} \sum_{k=0}^{n} \frac{i^{k}}{k !}(a d L)^{k}(X) \mathbb{E}\left(w(t)^{k}\right) \\
& =\lim _{n} \sum_{k=0}^{n} \frac{\left(-\frac{t}{2}\right)^{k}}{k !}(a d L)^{2 k}(X) \\
& =e^{-\frac{t}{2}(a d L)^{2}}(X),
\end{aligned}
$$

pues

$$
\begin{aligned}
\mathbb{E}\left(u(t)^{k}\right) & =\frac{1}{\sqrt{2 \pi}} \int_{-\infty}^{\infty} x^{k} e^{-\frac{x^{2}}{2 t} d x} \\
& =\left\{\begin{array}{lr}
0 & \text { si } k \text { es impar } \\
1 \cdot 3 \cdot 5 \cdots \cdot(2 m-1) l^{m} \text { si } k=2 m
\end{array}\right.
\end{aligned}
$$


entonces

$$
T_{t}(X)=e^{-\frac{t}{2}\left(a d L_{s}\right)^{2}}(X), t \geq 0 \text { y } X \in \mathcal{B}(\mathcal{H})
$$

De esto se sigue inmediatamente que $T_{0}(X)=X$ y $T_{l}(1)=1$, pues $($ ad $I)(1)=$ 0. Obsérvese además que

$$
\begin{aligned}
\left\|\mathbb{E}\left(e^{i w(t) L} X e^{-i w(t) L}\right)\right\| & \leq \mathbb{E}\left\|\left(e^{i w(t) L} X e^{-i w(t) L}\right)\right\| \\
& \leq \mathbb{E}\|X\|=\|X\|
\end{aligned}
$$

Por tanto, el semigrupo es le contracciones.

Por otra parte, si $X_{N} \underset{N}{\vec{w}} X$, entonces

$$
\begin{aligned}
\lim _{N}\left\langle u, T_{l}\left(X_{N}\right) v\right\rangle & =\lim _{N} \sum_{k=0}^{\infty} \frac{\left(-\frac{t}{2}\right)^{k}}{k !}\left\langle u,(a d L)^{2 k}\left(X_{N}\right) v\right\rangle \\
& =\sum_{k=0}^{\infty} \frac{\left(-\frac{t}{2}\right)^{k}}{k !} \lim _{N}\left\langle u,(a d L)^{2 k}\left(X_{N}\right) v\right\rangle \\
& =\sum_{k=0}^{\infty} \frac{\left(-\frac{t}{2}\right)^{k}}{k !}\left\langle u,(a d L)^{2 k}(X) v\right\rangle \\
& =\left\langle u, T_{t}(X) v\right\rangle,
\end{aligned}
$$

pues $\lim _{N}\left\langle u,(a d L)^{2 k}\left(X_{N}\right) v\right\rangle=\left\langle u,(a d L)^{2 k}(X) v\right\rangle$ y $\lim _{N}$ se intercambia con la serio porque se comple la estimación

$$
\left|\frac{\left(-\frac{l}{2}\right)^{k}}{k !}\left\langle u,(a d L)^{2 k}(X) v\right\rangle\right| \leq \frac{t^{k}}{k !} 2^{k}\|L\|^{2 k}\|X\|\|u\|\|v\| .
$$

Esto demuestra la propiedad (iii).

Tenemos arlemás que

$$
\begin{aligned}
\left\|\mathbb{E}\left(e^{i w(t) L} X e^{-i w(t) L}\right)-\mathbb{E}(X)\right\| & =\left\|\mathbb{E}\left[e^{i w(t) L} X e^{-i w(t) L}-X\right]\right\| \leq \\
& \leq\|X\| \mathbb{E}\left\|X e^{-i w(\ell) L}-1\right\|+\mathbb{E}\left\|e^{i w(t) L} X-X\right\| \\
& \leq\|X\| \mathbb{E}\left(\left\|X e^{-i w(t) L}-1\right\|+\mathbb{E}\left\|e^{i w(t) L}-1\right\|\right) \\
& \rightarrow 0,
\end{aligned}
$$

si $\iota \rightarrow 0$. Yo que implica arlemás que ol semigrupo es uniformemente contium) tomando el supremo sobre $\|X\| \leq 1$. 
Para verificar la propiedad (iv) de la definición de s.d.c. obsérvese que

$$
\begin{aligned}
\sum_{i, j}\left\langle Y_{i} \varphi, \mathbb{E}\left(e^{i w(t) L} X_{i}^{*} X_{j} e^{-i w(t) L}\right) Y_{j} \varphi\right\rangle & =\mathbb{E}\left(\sum_{i, j}\left\langle Y_{i} \varphi, e^{i w(t) L} X_{i}^{*} X_{j} e^{-i w(t) L} Y_{j} \varphi\right\rangle\right) \\
& =\mathbb{E}\left(\left\langle\sum_{i} X_{i} e^{-i w(t) L} Y_{i}, \sum_{i} X_{i} e^{-i w(t) L} Y_{i}\right\rangle\right) \\
& \geq 0
\end{aligned}
$$

pues podemos hacer uso del teorema de Fubini.

Finalmente, el generador de este semigrupo es

$$
\begin{aligned}
\frac{d}{d t} P_{t}(X) & \mid \quad t=0=-\frac{1}{2}(a d L)^{2}(X) \\
& =-\frac{1}{2}\left(L^{2} X+X L^{2}\right)+L X L \\
& =-\frac{1}{2}\left[L^{2}, X\right]_{+}+L X L
\end{aligned}
$$

Ejemplo 3: Sea $U$ un operador unitario en $\mathcal{H}$ y sea $\{N(t) \mid t \geq 0\}$ el proceso estocástico de Poisson con intensidarl $\lambda>0$, es decir, $N(t) \in \mathbb{N}, t \geq 0, N(t)$ tiene incrementos independientes y

$$
P(N(t)=k)=e^{-\lambda t} \frac{(\lambda t)^{k}}{k !} .
$$

\section{Definimos}

$$
T_{\iota}(X)=\mathbb{E} U^{* N(t)} X U^{N(\ell)}, t \geq 0, X \in \mathcal{B}(\mathcal{H})
$$

Entonces

$$
\begin{aligned}
T_{t+s}(X) & =\mathbb{E}\left(U^{* N(t+s)-N(s)} U^{* N(s)} X U^{N(s)} U^{N(t+s)-N(s)}\right) \\
& =T_{t}\left(T_{s}(X)\right)
\end{aligned}
$$

donde la esperanza se toma con respecto a la medida de Poisson en el mismo sentido del ejemplo anterior.

El generador se puede obtener de la siguiente manera

$$
\begin{aligned}
T_{l}(X) & =\sum_{k=0}^{\infty} \mathbb{E} 1_{\left\{N_{w}(t)=k\right\}} U^{* N_{w}(t)} X U^{N_{w}(l)} \\
& =X P(N(t)=0)+U^{*} X U P(N(t)=1)+U^{* 2} X U^{2} P(N(t)=2)+\cdots \\
& =X e^{-\lambda t}+U^{*} X U e^{-\lambda t} \lambda t+U^{* 2} X U^{2} e^{-\lambda \ell} \frac{(\lambda t)^{2}}{2 !}+\cdots
\end{aligned}
$$


entonces

$$
\begin{aligned}
\frac{d}{d t} T_{t}(X) & \mid \quad t=0=-\lambda X+\lambda U^{*} X U \\
& =\lambda\left(U^{*} X U-X\right)
\end{aligned}
$$

Que también se puede representar como

$$
\frac{d}{d t} T_{t}(X)=-\frac{1}{2}\left(L^{*} L X+X L^{*} L-2 L^{*} X L\right)
$$

$\operatorname{con} L=\sqrt{\lambda} U, L^{*}=\sqrt{\lambda} U^{*}$

De la representación

$$
T_{t}(X)=X e^{-\lambda t}+U^{*} X U e^{-\lambda t} \lambda t+U^{* 2} X U^{2} e^{-\lambda t} \frac{(\lambda t)^{2}}{2 !}+\cdots
$$

so obtiene facilmente que $T_{0}(X)=X, T_{l}(1)=1$. Tenemos

$$
\begin{aligned}
\left\|T_{l}(X)\right\| & =\left\|\mathbb{E} U^{* N(t)} X U^{N(t)}\right\| \\
& \leq \mathbb{E}\left\|U^{* N(t)} X U^{N(t)}\right\| \\
\mathbb{E}\|X\| & =\|X\| .
\end{aligned}
$$

Por otra parte,

$$
\begin{aligned}
\left\|\mathbb{E} U^{* N(t)} X U^{N(t)}-\mathbb{E} X\right\| & \leq \mathbb{E}\left(\left\|X U^{N(t)}-X\right\|+\left\|U^{* N(t)} X-X\right\|\right) \\
& \leq\|X\| \mathbb{E}\left(\left\|U^{N(t)}-1\right\|+\left\|U^{* N(t)}-1\right\|\right) \\
& \rightarrow 0,
\end{aligned}
$$

si $t \rightarrow 0$, lo cual demuestra la propiedad (ii) y además tomando el supreno solue $\|X\| \leq 1$ se observa que el semigrupo es uniformenente continuo.

La propiedad (iv) se sigue del hecho de que $T_{t}$ tiene la forma de Kraus (I.5). 


\section{Capítulo II}

\section{Teorema de}

\section{Gorini-Kossakowski-Sudarshan y Lindblad.}

Siguiendo a K.R.Parthasarathy $[P 2]$ en este capítulo desarrollamos una demostración del Teorema que le da nombre. La alternativa que se presenta aquí es, primeramente, la de no usar resultados de probabilidad en ninguna de las demostraciones, sino sólo teoremas del álgebra de operadores y del análisis funcional como son, la construcción de Gelfand-Naimark-Segal (GNS) y el teorema de representación de derivaciones, que se anexan en los apéndices. En segundo lugar, en la demostración de la suficiencia se sigue el método de A. Chebotarev $[C h 2],[G Q]$ para construir soluciones minimales de la ecuación maestra, lo que permite demostrar este resultado, sin utilizar la teoría de ecuaciones diferenciales estocásticas que Parthasarathy desarrolla en su libro.

Proposición 1: Sea 0 el generador de un semigrupo dinámico cuántico uniformemente continuo $\left\{T_{t}\right\}$ en $\mathcal{B}(\mathcal{H})$. Entonces se cumple lo siguiente:

(i) $\theta(1)=0$;

(ii) Si $X_{i}, Y_{i} \in \mathcal{B}(\mathcal{H}), 1 \leq i \leq n$ y $\sum_{i=1}^{n} X_{i} Y_{i}=0$ entonces

$$
\sum_{1 \leq i, j \leq n} Y_{i}^{*} \theta\left(X_{i}^{*} X_{j}\right) Y_{j} \geq 0
$$

(iii) Para todo $X_{i}, Y_{i} \in \mathcal{B}(\mathcal{H}), 1 \leq i \leq n$,

$$
\sum_{1 \leq i, j \leq n} Y_{i}^{*}\left\{\theta\left(X_{i}^{*} X_{j}\right)-\theta\left(X_{i}^{*}\right) X_{j}-X_{i}^{*} \theta\left(X_{j}\right)\right\} Y_{j} \geq 0
$$


Demostración: (i) Por la propiedad (iii) en la definición de s.d.c. $T_{t}(1)=1$ para toda $t$, así $\theta(1)=\lim _{t \rightarrow 0} t^{-1}\left(T_{t}(1)-1\right)=0$ donde el límite es en la norma de operadores.

(ii) De la propiedad (iv) en la definición de s.d.c. para todo $X_{i}, Y_{i} \in \mathcal{B}(\mathcal{H})$, $\sum_{1 \leq i, j \leq n} Y_{i}^{*} T_{t}\left(X_{i}^{*} X_{j}\right) Y_{j} \geq 0$ y por hipótesis $\sum_{i} X_{i} Y_{i}=0$, entonces

$$
\begin{aligned}
0 & \leq t^{-1} \sum_{1 \leq i, j \leq n} Y_{i}^{*} T_{t}\left(X_{i}^{*} X_{j}\right) Y_{j} \\
& =\sum_{1 \leq i, j \leq n} Y_{i}^{*} \ell^{-1}\left[T_{l}\left(X_{i}^{*} X_{j}\right)-X_{i}^{*} X_{j}\right] Y_{j}
\end{aligned}
$$

pues $\sum_{1 \leq i, j \leq n} Y_{i}^{*} X_{i}^{*} X_{j} Y_{j}=0$. Haciendo $t \rightarrow 0$ obtenemos (ii) ya que los operadores $T_{t}$ son contimuos en la norma de operadores.

(iii) Sean $X_{i}, Y_{i} \in \mathcal{B}(\mathcal{H}), 1 \leq i, j \leq n$ cualesquiera, póngase $X_{0}=1 \mathrm{y}$ $Y_{0}=-\sum_{i=1}^{n} X_{i} Y_{i}$ entonces

$\sum_{i=0}^{n} X_{i} Y_{i}=X_{0} Y_{0}+\sum_{i=1}^{n} X_{i} Y_{i}=0$. Por (ii) se obtiene

$$
\begin{aligned}
0 \leq & Y_{0}^{*} \theta\left(X_{0}^{*} X_{0}\right) Y_{0}+\sum_{j=1}^{n} Y_{0}^{*} \theta\left(X_{0}^{*} Y_{j}\right) Y_{j} \\
& +\sum_{i=1}^{n} Y_{i}^{*} \theta\left(X_{i}^{*} X_{0}\right) Y_{0}+\sum_{1 \leq i, j \leq n} Y_{i}^{*} \theta\left(X_{i}^{*} X_{j}\right) Y_{j} \\
= & \left(-\sum_{i=1}^{n} X_{i} Y_{i}\right)^{*} \theta(1)\left(-\sum_{i=1}^{n} X_{i} Y_{i}\right)+\sum_{j=1}^{n}\left(-\sum_{i=1}^{n} X_{i} Y_{i}\right)^{*} \theta\left(X_{j}\right) Y_{j} \\
& +\sum_{i=1}^{n} Y_{i}^{*} \theta\left(X_{i}^{*}\right)\left(-\sum_{j=1}^{n} X_{i} Y_{i}\right)+\sum_{1 \leq i, j \leq n} Y_{i}^{*} \theta\left(X_{i}^{*} X_{j}\right) X_{j} \\
= & -\sum_{i=1}^{n} Y_{i}^{*} X_{i}^{*} \sum_{j=1}^{n} \theta\left(X_{j}\right) Y_{j}-\sum_{i=1}^{n} Y_{i}^{*} \theta\left(X_{i}^{*}\right) \sum_{j=1}^{n} X_{j} Y_{j}+\sum_{j \leq i, j \leq n} Y_{i}^{*} \theta\left(X_{i} X_{j}\right) Y_{j} \\
= & \sum_{1 \leq i, j \leq n} Y_{i}^{*}\left\{\theta\left(X_{i}^{*} X_{j}\right)-\theta\left(X_{i}\right) X_{j}-X_{i}^{*} \theta\left(X_{j}\right)\right\} Y_{j} .
\end{aligned}
$$

En la siguiente Proposición se usa el teorema de construcción de GelfandNaimark-Segal CGNS cuyo enunciado y demostración se dan en el apéndice 1.

Proposición 2: Sea $\theta$ como en la Proposición 1. Entonces existen un espacio de Hilbert $\mathcal{K}$ y para todo $X \in \mathcal{B}(\mathcal{H})$, un operador lineal $\pi(X): \mathcal{H} \rightarrow$ $\mathcal{K}$ que satisfacen lo siguiente: 
(i) $\pi(X)^{*} \pi(Y)=\theta\left(X^{*} Y\right)-\theta\left(X^{*}\right) Y-X^{*} \theta(Y)$;

(ii) La correspondencia $X \rightarrow \pi(X)$ es lineal;

(iii) El conjunto $\{\pi(X) u \mid X \in \mathcal{B}(\mathcal{H}), u \in \mathcal{H}\}$ es total en $\mathcal{K}$;

(iv) Si $\left(\mathcal{K}^{\prime}, \pi^{\prime}\right)$ es otro par que satisface de (i) a (iii) entonces existe un isomorfismo unitario $\Gamma: \mathcal{K} \rightarrow \mathcal{K}^{\prime}$ tal que $\Gamma \pi(X) u=\pi^{\prime}(X)$ para todo $X$ $\in \mathcal{B}(\mathcal{H}), u \in \mathcal{H}$

Demostración: De la Proposición 1 se sigue que la función

$$
K((X, u),(Y, v))=\left\langle u,\left\{\theta\left(X^{*} Y\right)-\theta\left(X^{*}\right) Y-X^{*} \theta(Y)\right\} v\right\rangle
$$

es un Kernel definiclo positivo ${ }^{4}$ en el espacio $\mathcal{B}(\mathcal{H}) \times \mathcal{H}$, en efecto, por la propiedad (iii) de la Proposición 1 tenemos que

$$
\begin{aligned}
\sum_{1 \leq i, j \leq n} \bar{\alpha}_{i} \alpha_{j} K\left(\left(X_{i}, u_{i}\right),\left(X_{j}, u_{j}\right)\right) & =\sum_{1 \leq i, j \leq n} \bar{\alpha}_{i} \alpha_{j}\left\langle u_{i},\left\{\theta\left(X_{i}^{*} X_{j}\right)-\theta\left(X_{i}^{*}\right) X_{j}-X_{i}^{*} \theta\left(X_{j}\right)\right\} u_{j}\right\rangle \\
& =\sum_{1 \leq i, j \leq n}\left\langle\alpha_{i} u_{i},\left\{\theta\left(X_{i}^{*} X_{j}\right)-\theta\left(X_{i}^{*}\right) X_{j}-X_{i}^{*} \theta\left(X_{j}\right)\right\} \alpha_{j} u_{j}\right\rangle \\
& =\sum_{1 \leq i, j \leq n}\left\langle u, Y_{i}^{*}\left\{\theta\left(X_{i}^{*} X_{j}\right)-\theta\left(X_{i}^{*}\right) X_{j}-X_{i}^{*} \theta\left(X_{j}\right)\right\} Y_{j} u\right\rangle \\
& \geq 0,
\end{aligned}
$$

donde $Y_{i} u=\alpha_{i} u_{i}, u \in \mathcal{H}$ para toda $\alpha_{i} \in \mathbb{C}, u_{i} \in \mathcal{H}, i=1,2 \ldots, n$. Ahora, por el teorema construcción de Gelfand-Naimark-Segal ${ }^{5}$ existe un par de Gelfand $(\mathcal{K}, \lambda)$ donde $\mathcal{K}$ es un espacio de Hilbert y $\lambda: \mathcal{B}(\mathcal{H}) \times \mathcal{H} \rightarrow \mathcal{K}$ es una transformación tal que

$$
\langle\lambda(X, u), \lambda(Y, v)\rangle=\left\langle u,\left\{\theta\left(X^{*} Y\right)-\theta\left(X^{*}\right) Y-X^{*} \theta(Y)\right\} v\right\rangle
$$

para todo $u, v \in \mathcal{H}, X, Y \in \mathcal{B}(\mathcal{H})$, y el conjunto $\{\lambda(X, u) \mid X \in \mathcal{B}(\mathcal{H}), u \in \mathcal{H}\}$ es total en $\mathcal{K}$.

Dado que el lado derecho de (2.1) es antilineal conjugado en $(X, u)$ y lineal en $(Y, v)$ se sigue que $\lambda(X, u)$ es lineal en carla una de las variables $X, u$. Así, existe un operador $\pi(X): \mathcal{H} \rightarrow \mathcal{K}$ que satisface $\pi(X) u=\lambda(X, u)$ y $(2.1)$ implica $\|\pi(X) u\|^{2}=\left\langle u,\left\{\theta\left(X^{*} X\right)-\theta\left(X^{*}\right) X-X^{*} \theta(X)\right\} u\right\rangle$. Dado que $\theta$ es

\footnotetext{
${ }^{4}$ Ver apéndice 1

${ }^{5}$ Ver apéndice 1
} 
acotarlo se sigue que $\pi(X)$ es acotado, por la desigualdad de Schwartz, como operador de $\mathcal{H}$ en $\mathcal{K}$.

Ahora (2.1) implica que

(i) $\pi(X)^{*} \pi(Y)=\theta\left(X^{*} Y\right)-\theta\left(X^{*}\right) Y-X^{*} \theta(Y)$;

(ii) La lincalidad de $\pi$ se sigue de la de $\lambda$.

(iii) Esta propiedad es consecuencia del hecho de que $\{\lambda(X, u)\}$ es total.

(iv) Isa propiedad (iv) se sigue inmediatamente de la última parte del teorema de GNS.

Definición: Un homomorfismo $\Gamma: \mathcal{B}\left(\mathcal{H}_{1}\right) \rightarrow \mathcal{B}\left(\mathcal{H}_{2}\right)$ donde $\mathcal{H}_{1}, \mathcal{H}_{2}$ son espacios de Hilbert, se llama * ${ }^{*}$ unital si

(i) $\Gamma\left(X^{*}\right)=\Gamma^{*}(X)$.

(ii) $\Gamma\left(1_{\mathcal{H}_{1}}\right)=1_{\mathcal{H}_{2}}$.

Proposición 3: Sean $\theta, \pi$ como en la Proposición 2. Entonces existe un ho-

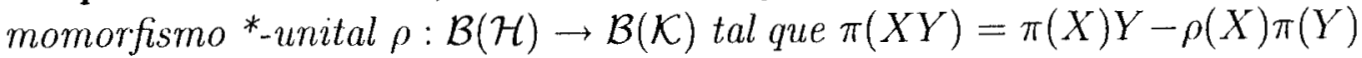
para todo $X, Y \in \mathcal{B}(\mathcal{H})$.

Demostración: Queremos definir $\rho: \mathcal{B}(\mathcal{H}) \rightarrow \mathcal{B}(\mathcal{K})$ para lo cual procedcremos definiendo o en $\mathcal{U}(\mathcal{H}) \subset \mathcal{B}(\mathcal{H})\left(U \in \mathcal{U}(\mathcal{H})\right.$ si y sólo si $\left.U U^{*}=1\right)$, seguidamente se define $\rho$ en combinaciones lineales finitas de unitarios, pnes cada $X \in \mathcal{B}(\mathcal{H})$ es combinación lineal de cuatro operadores unitarios ${ }^{6}$.

Sea $U \in \mathcal{U}(\mathcal{H})$ defínase

$$
\rho(U): k \rightarrow \mathcal{K}
$$

donde $k=\overline{\operatorname{span}\{\pi(X) u: X \in \overline{\mathcal{B}}(\mathcal{H}), u \in \mathcal{H}\}}$ mediante

$$
\rho(U) \pi(X) u=(\pi(U X)-\pi(U) X) u
$$

entonces

$$
\begin{aligned}
\langle\rho(U) \pi(X) u, \rho(U) \pi(Y) v\rangle= & \langle(\pi(U X)-\pi(U) X) u,(\pi(U Y)-\pi(U) Y) v\rangle \\
= & \langle\pi(U X) u, \pi(U Y) v\rangle-\langle\pi(U X) u, \pi(U) Y v\rangle \\
& -\langle\pi(U) X u, \pi(U Y) v\rangle+\langle\pi(U) X u, \pi(U) Y v\rangle \\
= & \left\langle u, \pi^{*}(U X) \pi(U Y) v\right\rangle-\left\langle u, \pi^{*}(U X) \pi(U) Y v\right\rangle \\
& -\left\langle u, X^{*} \pi^{*}(U) \pi(U Y) v\right\rangle+\left\langle u, X^{*} \pi(U)^{*} \pi(U) Y v\right\rangle
\end{aligned}
$$

${ }^{6}$ Ver apendice 5 


$$
\begin{aligned}
= & \left\langle u,\left\{\theta\left(X^{*} Y\right)-\theta\left(X^{*} U^{*}\right) U Y-X^{*} U^{*} \theta(U Y)\right\} v\right\rangle \\
& -\left\langle u,\left\{\theta\left(X^{*}\right)-\theta\left(X^{*} U^{*}\right) U-X^{*} U^{*} \theta(U)\right\} u\right\rangle \\
& -\left\langle u, X^{*}\left\{\theta(Y)-U^{*} \theta(U Y)-\theta\left(U^{*}\right) U Y\right\} v\right\rangle \\
& +\left\langle u, X^{*}\left\{-\theta\left(U^{*}\right) U-U^{*} \theta(U)\right\} Y v\right\rangle \\
= & \left\langle u,\left\{\theta\left(X^{*} Y\right)-\theta\left(X^{*}\right) Y-X^{*} \theta(Y)\right\} v\right\rangle \\
= & \left\langle u, \pi^{*}(X) \pi(Y) v\right\rangle \\
= & \langle\pi(X) u, \pi(Y) v\rangle
\end{aligned}
$$

donde la igualdad $\left(^{*}\right)$ se sigue de la Proposición 2 inciso (i).

Entonces, $\rho(U): k \rightarrow \mathcal{K}$ es isometría y se extiende a una isometría de $\mathcal{K}$ en sí mismo. Demostraremos ahora que $\rho\left(U^{*}\right)=\rho^{*}(U)$. Por definición

$$
\begin{aligned}
&\left\langle\rho(U)^{*} \pi(X) u, \pi(Y) v\right\rangle=\langle\pi(X) u, \rho(U) \pi(Y) v\rangle=\langle\pi(X) u,(\pi(U Y)-\pi(U) Y) v\rangle \\
&=\left\langle\pi(X) u,\left(\pi^{*}(X) \pi(U Y)-\pi^{*}(X) \pi(U) Y\right) v\right\rangle \\
&=\left\langle u,\left\{\theta\left(X^{*} U Y\right)-\theta\left(X^{*}\right) U Y-X^{*} \theta(U Y)\right\} v\right\rangle \\
&-\left\langle u,\left\{\theta\left(X^{*} U\right)-\theta\left(X^{*}\right) U-X^{*} \theta(U)\right\} Y v\right\rangle \\
&=\left\langle u,\left\{\theta\left(X^{*} U Y^{r}\right)-X^{*} \theta(U Y)-\theta\left(X^{*} U\right) Y+X^{*} \theta(U) Y\right\} u\right\rangle
\end{aligned}
$$

Por otra parte, tomando adjuntos y por la Proposición 2 inciso(i)

$$
\begin{aligned}
&\left\langle\rho\left(U^{*}\right) \pi(X) u, \pi(Y) v\right\rangle=\left\langle\pi\left(U^{*} X\right) u-\pi\left(U^{*}\right) X u, \pi(Y) v\right\rangle \\
&=\left\langle u, \pi\left(U^{*} X\right)^{*} u-X^{*} \pi\left(U^{*}\right)^{*} \pi(Y) v\right\rangle \\
&=\left\langle u,\left\{\theta\left(U^{*} X\right)^{*} Y-\theta\left(\left(U^{*} X\right)^{*}\right) Y-\left(U^{*} X\right)^{*} \theta(Y)\right\} v\right\rangle \\
&-\left\langle u, X^{*}\{\theta(U Y)-\theta(U) Y-U \theta(Y)\} v\right\rangle \\
&=\left\langle u,\left\{\theta\left(X^{*} U Y\right)-\theta\left(X^{*} U\right) y-X^{*} \theta(U Y)+X^{*} \theta(U) Y\right\} v\right\rangle
\end{aligned}
$$

$Y$ comparando obtenemos que $\rho\left(U^{*}\right)=\rho(U)^{*}$

Ahora, para cualesquiera dos operadores unitarios $U_{1}, U_{2} \in \mathcal{H}, \rho\left(U_{1} U_{2}\right)=$ $\rho\left(U_{1}\right) \rho\left(U_{2}\right)$ y $\rho(1)=1$. En efecto,

$$
\rho\left(U_{1} U_{2}\right) \pi(X) u=\pi\left(U_{1} U_{2} X\right) u-\pi\left(U_{1} U_{2}\right) X u
$$


$y$, por otra parte, como $\rho(X) \pi(Y)=\pi(X Y)-\pi(X) Y$ tenemos que

$$
\begin{aligned}
& \rho\left(U_{1}\right) \rho\left(U_{2}\right) \pi(X) u=\rho\left(U_{1}\right)\left[\pi\left(U_{2} X\right) u-\pi\left(U_{2}\right) X u\right] \\
= & \pi\left(U_{1} U_{2} X\right) u-\pi\left(U_{1}\right) U_{2} X u-\left(\pi\left(U_{1} U_{2}\right) X u-\pi\left(U_{1}\right) U_{2} X u\right) \\
= & \pi\left(U_{1} U_{2} X\right) u-\pi\left(U_{1} U_{2}\right) X u
\end{aligned}
$$

Lo cual demuestra que $\rho\left(U_{1} U_{2}\right)=\rho\left(U_{1}\right) \rho\left(U_{2}\right)$.

Además,

$$
\begin{aligned}
\rho(1) \pi(X) u & =\pi(1 X) u-\pi(1) X u \\
& =\pi(X) u=1
\end{aligned}
$$

pues $\pi(1)=0$, ya que $\pi^{*}(1) \pi(1) u=\left(\theta\left(1^{*} 1\right)-\theta\left(1^{*}\right) 1-1^{*} \theta(1)\right) u=0$.

Entonces, $\left\langle u, \pi^{*}(1) \pi(1) u\right\rangle=0$ para cada $u$ y por lo tanto $\langle\pi(1) u, \pi(1) u\rangle=$ 0 es decir $\pi(1) \equiv 0$. Esto demuestra que $\rho(1)=1$. Tenemos entonces que $\rho(U)$ es unitario para toda $U \in \mathcal{U}(\mathcal{H})$ pues $\rho^{*}(U) \rho(U)=\rho\left(U^{*} U\right)=\rho(1)=1$.

Ahora, si $X=\sum_{j} c_{j} U_{j}$ es combinación lineal finita de operadores en $\mathcal{H}$ se clefine

$$
\rho(X)=\sum_{j} c_{j} \rho\left(U_{j}\right)
$$

si $\sum c_{j} U_{j}=0$ entonces

$$
\sum c_{j} \rho\left(U_{j}\right) \pi(Y)=\pi\left(\sum c_{j} U_{j} Y\right)-\pi\left(\sum c_{j} U_{j}\right) Y=0
$$

es decir $\rho(X)$ está bien definida cuando $X$ es una combinación lincal de operadores unitarios, como todo operador en $\mathcal{B}(\mathcal{H})$ es combinación lineal de cuatro operadores unitarios $\rho(X)$ esta bien definido para todo $X$ en $\mathcal{B}(\mathcal{H})$. Esto implica que la transformación $X \rightarrow \rho(X)$ es un homomorfismo *-unital de $\mathcal{B}(\mathcal{H}) \rightarrow \mathcal{B}(\mathcal{K})$

Proposición 4: Sea $\rho: \mathcal{B}(\mathcal{H}) \rightarrow \mathcal{B}(\mathcal{K})$ el homomorfismo ${ }^{*}$-unital definido en la Proposición 3. Si w. lim $\lim _{n \rightarrow \infty} X_{n}=X$ en $\mathcal{B}(\mathcal{H})$ entonces

$$
\text { w. } \lim _{n+\infty} p\left(X_{n}\right) \cdots p\left(X^{\prime}\right) \cdot \| \mathcal{B}(\Lambda)
$$

Demostración: Sea w. $\lim _{n \rightarrow \infty} X_{n}=X$ en $\mathcal{B}(\mathcal{H})$ entonces por la propiedad (iii) en definición de s.d.e. tenemos

$$
\text { w. } \lim _{n \rightarrow \infty} T_{t}\left(X_{n}\right)=T_{t}(X), \forall t \geq 0
$$


es decir,

$$
\lim _{n \rightarrow \infty}\left\langle u, T_{t}\left(X_{n}\right) v\right\rangle=\left\langle u, T_{t}(X) v\right\rangle \forall u, v \in \mathcal{H}, t \geq 0
$$

entonces,

$$
\lim _{n \rightarrow \infty} \sum_{k=0}^{\infty} \frac{t^{k}}{k !}\left\langle u, \theta^{k}\left(X_{n}\right) v\right\rangle=\sum_{k=0}^{\infty} \frac{t^{k}}{k !}\left\langle u, \theta^{k}(X) v\right\rangle
$$

Poniendo

$$
\begin{aligned}
\varphi_{n}(t) & \left.=\sum_{k=2}^{\infty} \frac{t^{k-2}}{k !}\left\langle u, \theta^{k}\left(X_{n}\right) v\right\rangle\right\rangle \\
\varphi(t) & =\sum_{k=2}^{\infty} \frac{t^{k-2}}{k !}\left\langle u, \theta^{k}(X) v\right\rangle
\end{aligned}
$$

concluimos de la ecuación (4.1) derivando con respecto a $t$ que

$$
\lim _{n \rightarrow \infty}\left(\left\langle u,\left\{\theta\left(X_{n}\right)-\theta(X)\right\} v\right\rangle+t \varphi_{n}(t)-t \varphi(t)\right)=0, \forall t>0 .
$$

Por otra parte, dado que $\theta$ es acotado y $\sup _{n}\left\|X_{n}\right\|<\infty$ por el principio de acotamiento uniforme, existe $\alpha>0$ tal que

$$
\max \left(\left|\varphi_{n}(t)\right|,|\varphi(t)|\right)<e^{t r}
$$

pues,

$$
\begin{aligned}
\left|\varphi_{n}(t)\right| & \leq \sum_{k=2}^{\infty} \frac{t^{k-2}}{k !}\left|\left\langle u, \theta^{k}\left(X_{n}\right) v\right\rangle\right| \\
& \leq \sum_{k=2}^{\infty} \frac{t^{k}}{k !}\|u\| \beta\left\|X_{n}\right\|\|v\|, \text { para algím } \beta>0 \\
& \leq \sum_{k=2}^{\infty} \frac{t^{k}}{k ! !} \beta^{\prime}, \text { para algún } \beta^{\prime}>0 .
\end{aligned}
$$

similarmente para $|\varphi|$. Si $\varepsilon>0$ es arbitrario escogemos $t_{0}>0$ tal que $t_{0} e^{\alpha 2 t_{0}}<\frac{\varepsilon}{2}$, por la ec. (4.1) y (4.2) tenemos

$$
\begin{aligned}
\varlimsup_{n}\left|\left\langle u,\left\{\theta\left(X_{n}\right)-\theta(X)\right\} v\right\rangle\right| \leq & \varlimsup_{n}\left|\left\langle u,\left\{\theta\left(X_{n}\right)-\theta(X)\right\} v\right\rangle+t_{0} \varphi_{n}\left(t_{0}\right)-t_{0} \varphi\left(t_{0}\right)\right| \\
& +2 l_{0} c^{\prime t_{0}} \leq \varepsilon
\end{aligned}
$$

dado que $\varepsilon$ es arbitrario

$$
\lim _{n}\left\langle u, \theta\left(X_{n}\right) v\right\rangle=\langle u, \theta(X) v\rangle \vee v u, v \in \mathcal{H}
$$


Para todo $Y_{i} \in \mathcal{B}(\mathcal{H}), u_{i} \in \mathcal{H} \mathrm{i}=1,2$ tenemos de la definición de $\rho$ y $\pi$ en la Proposición 3 y la ecuación (4.3)

$$
\begin{aligned}
& \lim _{n \rightarrow \infty}\left\langle\pi\left(Y_{1}\right) u_{1,} \rho\left(X_{n}\right) \pi\left(Y_{2}\right) u_{2}\right\rangle= \lim _{n \rightarrow \infty}\left\langle\pi\left(Y_{1}\right) u_{1},\left\{\pi\left(X_{n} Y_{2}\right)-\pi\left(X_{n}\right) Y_{2}\right\} u_{2}\right\rangle \\
&= \lim _{n \rightarrow \infty}\left\langle u_{1}, \pi^{*}\left(Y_{1}\right) \pi\left(X_{n} Y_{2}\right)-\pi^{*}\left(Y_{1}\right) \pi\left(X_{n}\right) Y_{2} u u_{2}\right\rangle \\
&= \lim _{n \rightarrow \infty}\left\langle u_{1},\left\{\theta\left(Y_{1} X_{n} Y_{2}\right)-\theta\left(Y_{1}^{*}\right) X_{n} Y_{2}-Y_{1}^{*} \theta\left(X_{n} Y_{2}\right)\right\} u_{2}\right\rangle- \\
&-\lim _{n \rightarrow \infty}\left\langle u_{1},\left\{\left[\theta\left(Y_{1}^{*} X_{n}\right)-\theta\left(Y_{1}^{*}\right) X_{n}-Y_{1}^{*} \theta\left(X_{n}\right)\right] Y_{2}\right\} u_{2}\right\rangle \\
&=\left\langle u_{1},\left\{\theta\left(Y_{1} X Y_{2}\right)-\theta\left(Y_{1}^{*}\right) X Y_{2}-Y_{1}^{*} \theta\left(X Y_{2}\right)-\left[\theta\left(Y_{1}^{*} X\right)-\theta\left(Y_{1}^{*}\right) X-Y_{1}^{*} \theta(X)\right] Y_{2}\right\} u_{2}\right\rangle \\
&=\left\langle\pi\left(Y_{1}\right) u_{1}, \rho(X) \pi\left(Y_{2}\right) u_{2}\right\rangle
\end{aligned}
$$

Si $\|Y\|<1$ entonces,

$$
\left\|\frac{Y+Y^{*}}{2}\right\| \leq 1,\left\|\frac{Y-Y^{*}}{2 i}\right\| \leq 1
$$

y así

$$
\frac{Y+Y^{*}}{2}=\frac{U_{1}+U_{2}}{2}, \frac{Y-Y^{*}}{2 i}=\frac{V_{1}+V_{2}}{2}
$$

donde $U_{i}, V_{i}, \mathrm{i}=1,2$ son unitarios. Luego $\|\rho(Y)\| \leq 2$ cuando $\|Y\| \leq 1$. Entonces $\sup _{n}\left\|\rho\left(X_{n}\right)\right\|<\infty$.

Alora, de la tobalidad clel conjunto $\{\pi(Y) u \mid Y \in \mathcal{B}(\mathcal{H}), u \in \mathcal{H}\}$ en $\mathcal{K}$ y do la ecuación $(4.4)$ se oltiene que w. $\lim _{n} \rho\left(X_{n}\right)=\rho(X)$.

Proposición 5: Sea $\pi: \mathcal{B}\left(\mathcal{H}_{1}\right) \rightarrow \mathcal{B}(\mathcal{H})$ un homomorfismo*-unital. Existe un espacio de Hilbert $\mathcal{K}$ y un isomorfismo unitario $\Gamma: \mathcal{H} \rightarrow \mathcal{H}_{1} \otimes \mathcal{K}$ tal que $\pi(X)=\Gamma^{-1} X \otimes 1 \Gamma$ para toda $X \in \mathcal{B}\left(\mathcal{H}_{1}\right)$ donde 1 es el operador identidad en $\mathcal{K}$.

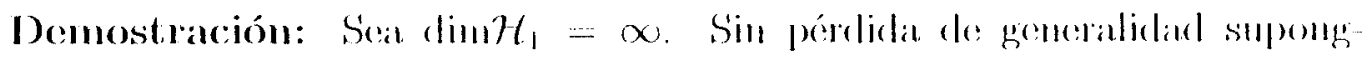
amos que $\mathcal{H}_{1}=\mathcal{L}^{2}(\mathbb{R})$ pues si $\mathcal{H}_{1}$ es separable, estos espacios son isomorfos. Tómense dos grupos uniparamétricos $U_{t}=e^{-i t_{q}}, V_{t}=e^{-i t p}$ donde $q, p$ satisfacen $[q, p]=i$. Sean $Q, P$ operadores autoadjuntos, generadores de $\pi\left(U_{l}\right), \pi\left(V_{l}\right)$, es decir, $\pi\left(U_{l}\right)=e^{-i t Q}, \pi\left(V_{l}\right)=e^{-i t P}$. Entonces $\pi\left(U_{l}\right), \pi\left(V_{l}\right)$, oberlecen las relaciones de commutación de Weyl, dado que $U_{s}, V_{t}$ satisfacen dicluas relaciones, es decin

$$
U_{s} V_{t}=e^{i s t} V_{t} U_{s}
$$


implica

$$
\begin{aligned}
\pi\left(U_{s}\right) \pi\left(V_{t}\right) & =\pi\left(U_{s} V_{t}\right) \\
& =\pi\left(e^{i s t} V_{l} U_{s}\right) \\
& =e^{i s t} \pi\left(V_{t} U_{s}\right) \\
& =e^{i s t} \pi\left(V_{t}\right) \pi\left(U_{s}\right)
\end{aligned}
$$

Por el teorema de Stone-von Nemmann ${ }^{7}$ existen $11 n$ espacio de Hilbert $\mathcal{K}$ y In isomorfismo mitario $\Gamma^{\prime}: \mathcal{H}_{1} \rightarrow \mathcal{B}(\mathbb{R}, \mathcal{K})$, es decir, de $\mathcal{H}_{1}$ en el conjunto de los operadores acotados de $\mathbb{R}$ en $\mathcal{K}$, tales que

$$
\begin{aligned}
& \left(\Gamma^{\prime} \pi\left(U_{s}\right) \Gamma^{\prime-1} f\right)(x)=e^{-i s \cdot x} f(x) \\
& \left(\Gamma^{\prime} \pi\left(V_{t}\right) \Gamma^{\prime-1} f\right)(x)=f(x-t)
\end{aligned}
$$

Entonces,

$$
\begin{aligned}
\left(\Gamma^{\prime} \pi\left(U_{s}\right) \Gamma^{\prime-1} \Gamma^{\prime} \pi\left(V_{t}\right) \Gamma^{\prime-1} f\right)(x) & =e^{-i s \cdot x}\left(\Gamma^{\prime} \pi\left(V_{t}\right) \Gamma^{\prime-1} f\right)(x) \\
& =e^{-i s \cdot x} f(x-t)
\end{aligned}
$$

pero dado que $U_{s}, V_{t}$ a su vez, satisfacen las relaciones de conmutación de Weyl, tenemos

$$
\left(\Gamma^{\prime} \pi\left(U_{s} V_{t}\right) \Gamma^{\prime-1} f\right)(x)=U_{s} V_{i} f(x)
$$

y así, obtenemos

$$
\pi\left(U_{s} V_{t}\right)=\Gamma^{-1}\left(U_{s} V_{t} \otimes 1\right) \Gamma
$$

donde $\Gamma=\Gamma^{-1}$. Por el isomorfismo entre el espacio ${ }^{\gamma} \mathcal{L}^{2}\left(\mathcal{H}_{1}, d \mu\right) \otimes \mathcal{K}$ y $\mathcal{L}^{2}\left(\mathcal{H}_{1}, d \mu ; \mathcal{K}\right)$. Como las combinaciones lineales de $U_{s}, V_{t}$, son densas en sentido débil ${ }^{9}$ en $\mathcal{B}(\mathcal{H})$ se sigue que $\pi(X)=\Gamma^{-1}(X \otimes 1) \Gamma$.

Ahora, supongamos que dim $\mathcal{H}_{1}=n<\infty$. Entonces, salvo equivalencia unitaria, existe un par irreducible de operadores unitarios $U, V \in \mathcal{H}_{1}$ tales que $U^{n}=V^{n}=1$ y $U V=\exp \left(\frac{2 \pi i}{n}\right) V U$. Por un análogo al teorema de Stone-von Neumann para el grupo $\{0,1,2, \ldots, n-1\}$ con adición módulo $n$ existe un espacio de Hibert $\mathcal{K}$ tal que $\Gamma^{-1}(U \otimes 1) \Gamma=\pi(U), \Gamma^{-1}(V \otimes 1) \Gamma=\pi(V)$.

\footnotetext{
7 Ver apéndice 2

${ }^{8} \mathcal{C}^{2}\left(\mathcal{H}_{1}, d_{\mu} ; \mathcal{K}\right)$ es el conjunto de todas las funciones medibles $f$ de $\mathcal{H}_{1}$ en $\mathcal{K}$ que satisfacen $\int_{\mathcal{H}_{1}}\|f(x)\|_{\mathcal{K}}^{2} d \mu(x)<\infty$.

${ }^{9}$ Para una demostración de este hecho, ver el libro de P.A. Meyer, $[M]$, Cap). 3 Sec. 6
} 
El álgebra generada por $U, V$ es $\mathcal{B}\left(\mathcal{H}_{1}\right)$ y, por el mismo argumento del caso $n=\infty$ tenemos $\pi(X)=\Gamma^{-1}(X \otimes 1) \Gamma$.

Corolario 1: Sea $\theta$ el generador del s.d.c uniformemente continuo $\left\{T_{t}\right\}$ en $\mathcal{B}(\mathcal{H})$. Entonces existe un espacio de Hilbert $\mathcal{K}$ y una función lineal $\pi$ : $\mathcal{B}(\mathcal{H}) \rightarrow \mathcal{B}(\mathcal{H} \otimes \mathcal{K})$ tal que

(i) $\{\pi(X) u \mid X \in \mathcal{B}(\mathcal{H}), u \in \mathcal{H}\}$ es total en $\mathcal{H} \otimes \mathcal{K}$

(ii) $\pi(X)^{*} \pi(Y)=\theta\left(X^{*} Y\right)-\theta\left(X^{*}\right) Y-X^{*} \theta(Y)$;

(iii) $\pi(X Y)=\pi(X) Y+(X \otimes 1) \pi(Y)$ para todo $X, Y \in \mathcal{B}(\mathcal{H})$.

Demostración: Sean $\theta, \pi, \rho$ como en la Proposición 3. Por las Proposiciones 4 y 5 existe un espacio de Hilbert $\mathcal{K}_{1}$ y un isomorfismo mitario $\Gamma: \mathcal{K} \rightarrow$ $\mathcal{H} \otimes \mathcal{K}_{1}$ tal que $\rho(X)=\Gamma^{-1} X \otimes 1 \Gamma$ para toda $X$ en $\mathcal{B}(\mathcal{H})$. Defínase $\pi_{0}(X)=$ $\Gamma \pi(X)$. Entonces se cumplen las propiedades (i) a (iii), con $\pi$ reemplazado por $\pi_{0}$, por la Proposición 2 y la definición de $\rho$, ya que $\Gamma$ es isomorfismo $y$ $\{\pi(X) u\}$ es total en $\mathcal{K}$ y por lo tanto $\Gamma \pi$ es total en $\mathcal{H} \otimes \mathcal{K}$ y se cumple (i).

La propiedarl (ii) se cmmple porque

$$
\begin{aligned}
\pi_{0}(X)^{*} \pi_{0}(Y) & =(\Gamma \pi(X))^{*} \Gamma \pi(Y) \\
& =\pi(X)^{*} \Gamma^{*} \Gamma \pi(Y) \\
& =\pi(X)^{*} \pi(Y)
\end{aligned}
$$

ya que $\Gamma^{*} \Gamma=1$ por ser $\Gamma$ unitario.

La propiedad (iii) es consecuencia de

$$
\begin{aligned}
\pi_{0}(X Y) & =\Gamma \pi(X Y) \\
& =\Gamma[\pi(X) Y+\rho(X) \pi(Y)] \\
& =\Gamma \pi(X) Y+\Gamma\left[\Gamma^{-1}(X \otimes 1) \Gamma \pi(Y)\right] \\
& =\pi_{0}(X) Y+(X \otimes 1) \pi_{0}(Y)
\end{aligned}
$$

Proposición 6: Sea 0 el generador de un s.d.c. uniformente continuo $\left\{T_{l}\right\}$ en $\mathcal{B}(\mathcal{H})$. Entonces existe una sucesión $\left\{L_{j}\right\}$ en $\mathcal{B}(\mathcal{H})$ que satisface lo siguiente.

(i)

$$
\operatorname{sip}_{\substack{\|u\| \leq 1 \\\|x\| \leq 1}} \sum_{j}\left\|\left[L_{j}, X\right] u\right\|^{2}<\infty
$$

(ii)

$$
\theta\left(X^{*} Y\right)-\theta\left(X^{*}\right) Y-X^{*} \theta(Y)=\sum_{j}\left[L_{j}, X\right]^{*}\left[L_{j}, Y\right]
$$


para todo $X, Y$ en $\mathcal{B}(\mathcal{H})$, donde la suma del lado derecho converge fuertemente.

Demostración: En el Corolario 1 sea $\left\{e_{j}\right\}_{j \geq 1}$ una base ortonormal en $\mathcal{K} y$ considérese

$$
\Gamma_{0}: \mathcal{H} \otimes \mathcal{K} \rightarrow \mathcal{H} \oplus \mathcal{H} \oplus \cdots
$$

tal que $\Gamma_{0} u \otimes v=\oplus_{j}\left\langle e_{j}, v\right\rangle u$ para todo $v \in \mathcal{K}, u \in \mathcal{H}$. Probaremos que $\Gamma_{0}$ es isomorfismo mitario:

$$
\begin{aligned}
\left\langle\Gamma_{0} u \otimes v, \Gamma_{0} u^{\prime} \otimes v^{\prime}\right\rangle & =\left\langle\underset{j}{\oplus}\left\langle e_{j}, v\right\rangle u, \oplus_{j}^{\oplus}\left\langle e_{j}, v^{\prime}\right\rangle u^{\prime}\right\rangle \\
& =\sum_{j} \overline{\left\langle e_{j}, v\right\rangle}\left\langle e_{j}, v^{\prime}\right\rangle\left\langle u, u^{\prime}\right\rangle \\
& =\left\langle v, v^{\prime}\right\rangle\left\langle u, u^{\prime}\right\rangle \\
& =\left\langle u \otimes v, u^{\prime} \otimes v^{\prime}\right\rangle,
\end{aligned}
$$

pues $\left\langle v, v^{\prime}\right\rangle=\sum \overline{\left\langle e_{j}, v\right\rangle}\left\langle e_{j}, v^{\prime}\right\rangle$.

Para cualcuier $X \in \mathcal{B}(\mathcal{H})$ existen $\pi_{j} \in \mathcal{B}(\mathcal{H})$ tales coue $\Gamma_{0} \pi(X) u=$ $\oplus_{j} \pi_{j}(X) u, u \in \mathcal{H}$. Entonces

$$
\pi_{j}(X Y) u=\pi_{j}(X) Y u+X \pi_{j}(Y) u,
$$

pries,

$$
\pi(X Y) u=\pi(X) Y u+(X \otimes 1) \pi(Y) u,
$$

lo cual implica que

$$
\begin{aligned}
\oplus_{j}^{\oplus} \pi_{j}(X Y) & =\Gamma_{0} \pi(X) Y u+\Gamma_{0}(X \otimes 1) \pi(Y) u \\
& =\underset{j}{\oplus} \pi_{j}(X) Y u+\Gamma_{0}(X \otimes 1)(w \otimes v),
\end{aligned}
$$

$\operatorname{con} \pi(Y) u=w \otimes v$ tenemos

$$
\begin{aligned}
\underset{j}{\oplus} \pi_{j}(X Y) & =\oplus_{j} \pi_{j}(X) Y u+\Gamma_{0} X u \otimes 1 v \\
& =\underset{j}{\oplus} \pi_{j}(X) Y u+\underset{j}{\oplus}\left\langle e_{j}, v\right\rangle X u \\
& =\underset{j}{\oplus} \pi_{j}(X) Y u+\underset{j}{\oplus} X\left(\left\langle e_{j}, v\right\rangle w\right) \\
& =\underset{j}{\oplus}\left(\pi_{j}(X) Y u+X \pi_{j}(Y) u\right),
\end{aligned}
$$


entonces $\pi_{j}$ es una derivación acotada ${ }^{10}$ en $\mathcal{B}(\mathcal{H})$ para carla $j$. Por lo tanto existen $L_{j}$ en $\mathcal{B}(\mathcal{H})$ tales que $\pi_{j}(X)=\left[L_{j}, X\right]$.

Ahora, $\Gamma_{0}$ es unitario y $\pi$ acotado por lo tanto $\Gamma_{0} \pi$ es acotado así que.

$$
\begin{aligned}
\infty & >\left\|\Gamma_{0} \pi(X) u\right\|^{2}=\left\|\underset{j}{\oplus} \pi_{j}(X) u\right\|^{2} \\
& =\sum_{j}\left\|\pi_{j}(X) u\right\|^{2} \\
& =\sum_{j}\left\|\left[L_{j}, X\right] u\right\|^{2}
\end{aligned}
$$

Para cada $u \in \mathcal{H}$ y $X \in \mathcal{B}(\mathcal{H})$. Tomando supremos obtenemos que

$$
\sup _{\substack{\|u\| \leq 1 \\\|X\| \leq 1}} \sum_{j=1}^{\infty}\left\|\left[L_{j}, X\right] u\right\|^{2} \leq \sup _{\substack{\|u\| \leq 1 \\\|X\| \leq 1}}\left\|\Gamma_{0} \pi_{j}(X) u\right\|^{2}<\infty .
$$

La propiedad (ii) se sigue de la propiedad (ii) del Corolario 1.

Proposición 7: Sea $\left\{L_{j}\right\}$ una sucesión en $\mathcal{B}(\mathcal{H})$ que satisface (6.1) entonces

$$
\sup _{\|u\|=1} \sum_{j}\left(\left\|L_{j} u\right\|^{2}-\left|\left\langle u, L_{j} u\right\rangle\right|^{2}\right)<\infty
$$

Demostración: En la ecuación (6.1) ponemos $X=|u\rangle\langle u|$ y observamos que

$$
\begin{gathered}
\left\|\left[L_{j}, X\right] u\right\|^{2}=\| L_{j}|u\rangle\langle u|u-| u\rangle\langle u| L_{j} u \|^{2} \\
=\left\langle L_{j} \mid u\right\rangle\langle u|u-| u\rangle\left\langle u\left|L_{j} u, L_{j}\right| u\right\rangle\langle u|u-| u\rangle\left\langle u \mid L_{j} u\right\rangle \\
=\left\langle L_{j}\|u\|^{2} u, L_{j}\|u\|^{2} u\right\rangle-\left\langle L_{j}\|u\|^{2} u,\left\langle u, L_{j} u\right\rangle u\right\rangle-\left\langle\left\langle u, L_{j} u\right\rangle u, L_{j} u\right\rangle \\
+\langle\mid u\rangle\left\langle u\left|L_{j} u,\right| u\right\rangle\left\langle u \mid L_{j} u\right\rangle,
\end{gathered}
$$

pues $|u\rangle\langle u| u=\|u\|^{2} u$. Y como $\|u\|^{2}=1$, tenemos que

$$
\begin{aligned}
& \left\|\left[L_{j}, X\right] u\right\|^{2}=\left\|L_{j} u\right\|^{2}-\left\langle L_{j} u,\left\langle u, L_{j} u\right\rangle u\right\rangle- \\
& -\left\langle\left\langle u, L_{j} u\right\rangle u, L_{j} u\right\rangle+\left\langle\left\langle u, L_{j} u\right\rangle u,\left\langle u, L_{j} u\right\rangle u\right\rangle \\
& =\left\|L_{j} u\right\|^{2}-\left\langle u, L_{j} u\right\rangle\left\langle L_{j} u, u\right\rangle- \\
& -\overline{\left\langle u, L_{j} u\right\rangle}\left\langle u, L_{j} u\right\rangle+\left\langle u, L_{j} u\right\rangle \overline{\left\langle u, L_{j} u\right\rangle} \\
& =\left\|L_{j} u\right\|^{2}-\left|\left\langle u, L_{j} u\right\rangle\right|^{2}
\end{aligned}
$$

\footnotetext{
"Ver apéndice 3.
} 
Proposición 8: Sea $\left\{L_{j}\right\}$ una sucesión en $\mathcal{B}(\mathcal{H})$ que satisface $(6.1)$ enlonces para cualquier proyección $P$ en $\mathcal{H}$

$$
\operatorname{sul}_{\substack{\|X\| \leq \leq \leq \\\|u\|=1, P u=u}} \sum_{j}\left\|\left[P L_{j} P, P X P\right] u\right\|^{2}<\infty
$$

Demostración: Cuando $P u=u$ tenemos

$$
\begin{aligned}
{\left[P L_{j} P, P X P\right] \|=} & \left(P L_{j} P P X P-P X P P L_{j} P\right) u \\
& \left(I l_{j} I^{\prime} X I^{\prime} I X I l^{\prime} l^{\prime}\right) u \\
= & P\left(L_{j} P X-X P L_{j}\right) P u \\
= & P\left(L_{j} P X-X P L_{j}\right) u
\end{aligned}
$$

por otra parte,

$$
\begin{aligned}
P\left[L_{j}, P X P\right] u & =P\left(L_{j} P X P-P X P L_{j}\right) u \\
& =P\left(L j P X P u-X P L_{j}\right) u \\
& =P\left(L_{j} P X-X P L_{j}\right) u
\end{aligned}
$$

pues $P u=u$, entonces

$$
\begin{aligned}
\left\|\left[P L_{j} P, P X P\right] u\right\| & =\left\|P\left[L_{j}, P X P\right] u\right\| \\
& \leq\left\|\left[L_{j}, P X P\right] u\right\| .
\end{aligned}
$$

El resultado se sigue de la ecuación (6.1).

Proposición 9: Sea dim $\mathcal{H}=n<\infty$. Denotamos por dU la diferencial de la medida de Haar ${ }^{11}$ normalizada del grupo compacto $\mathcal{U}(\mathcal{H})$. Entonces existen constantes positivas $\alpha, \beta$ lales que $\alpha+n \beta=n^{-1} y$

(i) $\int\|L U u\|^{2} d U=n^{-1} \operatorname{lr} L^{*} L$;

(ii) $\int\left|\left\langle u, U^{*} L U u\right\rangle\right|^{2} d U=\alpha \operatorname{tr} L^{*} L+\beta|\operatorname{tr} L|^{2}$ para todo $L$ en $\mathcal{B}(\mathcal{H})$ y lodo vector unitario $u$ en $\mathcal{H}$.

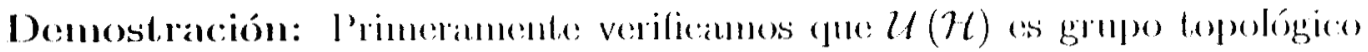
con la topología fuerte. Efectivamente, si $\left\{T_{n}\right\},\left\{S_{n}\right\}$, son sucesiones de operalores unitarios que convergen en la topología fuerte (darlo que $\mathcal{H}$ es

\footnotetext{
1 Ver apéndice, 4.
} 
de dimensión finita, la topología fuerte y la débil son equivalentes) a $T, S \in$ $\mathcal{U}(\mathcal{H})$ respectivamente, entonces,

$$
T_{n}^{*} \stackrel{s}{\rightarrow} T^{*}
$$

pero $T_{n}^{*}=T_{n}^{-1}$ y $T^{*}=T^{-1}$ por ser operadores unitarios. Por tanto

$$
T_{n}^{-1} \stackrel{s}{\rightarrow} T^{-1}
$$

Por otra parte,

$$
\begin{aligned}
\left\|\left(S_{n} T_{n}-S T\right) \varphi\right\| & \leq\left\|S\left(T_{n}-T\right) \varphi\right\|+\left\|\left(S_{n}-S\right) T \varphi\right\| \\
& \leq\left\|\left(T_{n}-T\right) \varphi\right\|+\left\|\left(S_{n}-S\right) T \varphi\right\| \rightarrow 0 .
\end{aligned}
$$

Como $\|T\| \leq 1$ para todo $T \in \mathcal{U}(H)$, entonces $\mathcal{U}(\mathcal{H})$ es compacto $(\operatorname{dim} \mathcal{H}=n<\infty)$. En particular, $\mathcal{U}(\mathcal{H})$ es localmente compacto y por tanto existe en éste, una única medida de Haar regular.

Por la invarianza bilateral de la medida de Haar de $\mathcal{U}(\mathcal{H})$ el lado izquierdo le (i) es independiente del vector $u$. Así, para cualquier base ortogonal $e_{1}, \ldots, e_{n}$, en $\mathcal{H}$ tenemos

$$
\int\|L U u\|^{2} d U=n^{-1} \sum_{j=1}^{n} \int\left\|L U e_{j}\right\|^{2} d U
$$

pues los $e_{j}$ son unitarios y la medida no depende del unitario elegido, además,

$$
\begin{aligned}
\frac{1}{n} \sum_{j}\left\langle L U e_{j}, L U e_{j}\right\rangle & =\frac{1}{n} \sum_{j=1}^{n}\left\langle U e_{j}, L^{*} L U e_{j}\right\rangle \\
& =\frac{1}{n} \operatorname{tr}\left(L^{*} L\right),
\end{aligned}
$$

por lo lanto

$$
\begin{aligned}
\int\|L U u\|^{2} d U & =\frac{1}{n} \int \operatorname{tr}\left(L^{*} L\right) d U \\
& =\frac{1}{n} \operatorname{lr}\left(L^{*} L\right)
\end{aligned}
$$

pues $\int d U=1$ y $U e_{j}$ es una base de $\mathcal{H}$ y la traza es independiente de la base elegicla. Para probar (ii) consiclérese la forma sesquilincal

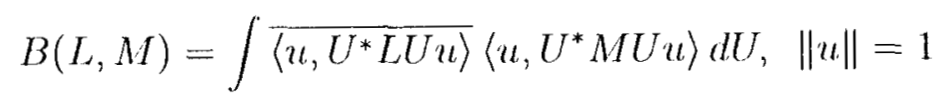


an el espacio $\mathcal{B}(\mathcal{H})$. Enlonces

$$
B\left(U^{*} L U, U^{*} M U\right)=B(L, M), \forall L, M \in \mathcal{B}(\mathcal{H}), U \in \mathcal{H}(\mathcal{H})
$$

por la invarianza de la medida de Haar.

Por el argumento en la pricha de (i)

$$
B(L, 1)=n^{-1} \operatorname{tr} L
$$

Para cualesquiera proyecciones ortogonales $P, Q$ tales que $P Q=0$ póngase

$$
a=B(P, P), b=B(P, Q)
$$

por (9.1) éstas son constantes positivas independientes de $P, Q$ ya que si $P^{\prime}, Q^{\prime}$ son proyecciones ortogonales entonces existe $U \in \mathcal{U}(\mathcal{H})$ tal que $P=$ $U^{*} P^{\prime} U$, similarmente para $Q$. ( Si $P, Q$ son proyecciones ortogonales en $\mathcal{H}$ tales que $\|P-Q\|<1$ entonces, $P$ y $Q$ son unitariamente equivalentes $\left.{ }^{12}\right)$. Sea $1=P_{1}+\cdots+P_{n}$ dado que $\operatorname{Tr} P_{k}=1, B(P, 1)=n^{-1}$ por (9.2). Por otra parte si $1=P+Q_{1}+\cdots+Q_{n-1}$ donde $P, Q_{j}$ son proyecciones ortogonales, entonces

$$
\begin{aligned}
B(P, 1) & =B\left(P, P+Q_{1}+\cdots+Q_{n-1}\right) \\
& =B(P, P)+B\left(P, Q_{1}\right)+\cdots+B\left(P, Q_{n-1}\right) \\
& =B(P, P)+(n-1) B(P, Q)
\end{aligned}
$$

donde $Q$ es cualquiera de las $Q_{i}$, así $a+(n-1) b=n^{-1}$ donde $a=B(P, P), b=$ $B(P, Q)$. Por la designaldad de Schwarz

$$
\begin{aligned}
b & =\int\left\langle u, U^{*} P U u\right\rangle\left\langle u, U^{*} Q U u\right\rangle d U \\
& \leq\left\{\int\left\langle u, U^{*} P U\right\rangle^{2} \int\left\langle u, U^{*} Q U\right\rangle^{2}\right\}^{\frac{1}{2}}=a
\end{aligned}
$$

Para todo $L$ autoadjunto con resolución espectral $L=\sum \lambda_{j} P_{j}$ donde cada. $P_{j}$ es una proyección unidimensional tenemos

$$
B(L, L)=\int \overline{\left.\left\langle u, U^{*} L U \|\right\rangle\right\rangle}\left\langle u, U^{*} L U \cdot u\right\rangle
$$

\footnotetext{
12Ver Weidmann [W], Teorema 4.35 pag 86.
} 


$$
\begin{aligned}
= & \int\left\langle u, U^{*}\left(\sum_{j} \lambda_{j} L_{j}\right) U u\right\rangle\left\langle u, U^{*}\left(\sum_{j} \lambda_{j} L_{j}\right) U u\right\rangle d U \\
= & B\left(\sum \lambda_{j} P_{j}, \sum \lambda_{j} P_{j}\right) \\
= & \sum \overline{\lambda j} B\left(P_{j}, \sum \lambda_{j} P_{j}\right) \\
& \sum_{j} \lambda_{j}^{2} I^{\prime}\left(I_{j}^{\prime}, I_{j}^{\prime}\right) \mid \sum_{j \neq k} \lambda_{j} \lambda_{k} I^{\prime}\left(I_{j}^{\prime}, I_{k}\right) \\
= & a \sum \lambda_{j}^{2}+b \sum_{j \neq k} \lambda_{j} \lambda_{k} \\
= & a t r L^{2}+\frac{b}{2}\left\{(t r L)^{2}-\operatorname{lr} L^{2}\right\} .
\end{aligned}
$$

Para cada par $I, M$, de operadores hermitianos tenemos

$$
B(L, M)=\frac{1}{4}\{B(L+M, L+M)-B(L-M, L-M)\}
$$

pues,

$$
\begin{aligned}
B(L, M)= & \frac{1}{4}\{B(L, L)+B(L ; M)+B(M, L)+B(M, M)\} \\
& -\frac{1}{4}(B(L, L)-B(L, M)-B(M, L)+B(M, M))
\end{aligned}
$$

ya que $L$ y $M$ son hermitianos y además

$$
\begin{aligned}
\left\langle u, U^{*} L U u\right\rangle & =\langle U u, L U u\rangle \\
& =\langle L U u, U u\rangle \\
& =\left\langle U^{*} L U u, u\right\rangle \\
& =\frac{\left\langle u, U^{*} L U u\right\rangle}{}
\end{aligned}
$$

de donde se sigue la igualdad $B(L, M)=B(M, L)$.

Por (9.3) y (9.4) tenemos

$$
\begin{aligned}
B(L, M)= & \frac{1}{4}\left\{(a-b) \operatorname{tr}(L+M)^{2}+b(\operatorname{tr}(L+M))^{2}\right\} \\
& -\frac{1}{4}\left\{(a-b) \operatorname{tr}(L-M)^{2}+b(\operatorname{tr}(L-M))^{2}\right\} \\
= & (a-b) \operatorname{tr} L M+b \operatorname{tr} L \operatorname{tr} M
\end{aligned}
$$

para $L, M$ arbitrarios $L=L_{1}+i L_{2}, M=M_{1}+i M_{2}$ donde $L_{i}, M_{i}$ son hermitianos

$$
B(L, M)=B\left(L_{1}+i L_{2}, M_{1}+i M_{2}\right)
$$




$$
\begin{gathered}
=(a-b)\left\{\operatorname{tr} L_{1} M_{1}+i \operatorname{tr} L_{1} M_{2}-i \operatorname{tr} L_{2} M_{1}+\operatorname{tr} L_{L_{2}} M_{2}\right\}+ \\
+b\left\{\operatorname{tr} L_{1} \operatorname{tr} M_{1}+i \operatorname{tr} L_{1} \operatorname{tr} M_{2}-i \operatorname{tr} L_{2} \operatorname{tr} M_{1}+\operatorname{tr} L_{\mu_{2}} \operatorname{tr} M_{2}\right\} \\
=(a-b) \operatorname{tr} L^{*} M+b \overline{\operatorname{tr} L} \operatorname{tr} M
\end{gathered}
$$

Poniendo $\alpha=a-b, \beta=\frac{b}{2}, L=M$ y usando la relación $a+(n-1) b=n^{-1}$ tememos (ii) donde $\alpha, \beta$ som positivos y $\alpha+n \beta=\frac{1}{n}$.

Proposición 10: $\operatorname{Sea} \operatorname{dim} \mathcal{H}=n<\infty$ y sea $\left\{L_{j}\right\}$ una sucesión en $\mathcal{B}(\mathcal{H})$ que satisface (6.1). Entonces

$$
\sum_{j} l r\left(L_{j}-\frac{1}{n} t r L_{j}\right)^{*}\left(L_{j}-\frac{1}{n} \operatorname{tr} L_{j}\right)<\infty
$$

Demostración: Por las Proposiciones 7 y 9

$$
\int \sum_{j}\left\{\left\|L_{j} U u\right\|^{2}-\left|\left\langle u, U^{*} L_{j} U u\right\rangle\right|^{2}\right\} d U<\infty
$$

ya (que)

$$
\int\left\|L_{j} U u\right\|^{2} d U=\frac{1}{n} \operatorname{tr} L^{*} L
$$

y

$$
\int \mid\left\langle u,\left.J^{*} L_{j}(U u\rangle\right|^{2} d U I=\alpha \operatorname{lr} l_{j}^{*} L_{j}+\beta\left|l \cdot r L_{, j}\right|^{2}\right.
$$

Entonces, sustituyendo estos valores en (10.1) obtenemos

$$
\sum_{j}\left(\frac{1}{n} \operatorname{tr} L_{j}^{*} L_{j}-\alpha \operatorname{tr} L_{j}^{*} L_{j}-\beta\left|\operatorname{tr} L_{j}\right|^{2}\right)<\infty
$$

además $n \beta=n^{-1}-\alpha$ y así

$$
\begin{aligned}
\sum_{j} n \beta\left(\operatorname{tr} L_{j}^{*} L_{j}\right)-\beta\left|\operatorname{tr} L_{j}\right|^{2} & =n \beta \sum_{j}\left(\operatorname{tr} L_{j}^{*} L_{j}-\frac{1}{n}\left|\operatorname{tr} L_{j}\right|^{2}\right) \\
& =n \beta \sum_{j}\left(\operatorname{tr}\left(L_{j}^{*} L_{j}\right)-\frac{1}{n} \overline{\operatorname{tr} L_{j}} \operatorname{tr} L_{j}\right) \\
& =n \beta \sum_{j}\left(\operatorname{tr}\left(L_{j}^{*} L_{j}\right)-\frac{1}{n} \operatorname{tr} L_{j}^{*} \operatorname{tr} L_{j}\right)
\end{aligned}
$$


darlo que $\operatorname{tr} I=n$

$$
\begin{aligned}
& =n \beta \sum_{j} \operatorname{tr}\left(L_{j}^{*} L_{j}-\frac{1}{n}\left(\operatorname{tr} L_{j}^{*}\right) L_{j}-\frac{1}{n} L_{j}^{*} \operatorname{tr} L_{j}+\frac{1}{n^{2}} \operatorname{tr} L_{j}^{*} \operatorname{tr} L_{j}\right) \\
& =n \beta \sum_{j} \operatorname{tr}\left(L_{L_{j}}-\frac{1}{n} \operatorname{tr} L_{j}\right)^{*}\left(L_{j}-\frac{1}{n} \operatorname{lr} L_{j}\right)<\infty
\end{aligned}
$$

pues $\left(\left(\operatorname{tr} L_{j}\right) 1\right)^{*}=\overline{\operatorname{tr}} \bar{L}_{j} 1=\operatorname{tr} L_{j}^{*} 1$.

Proposición 11: Sea $\mathcal{H}$ un espacio de Hilbert y sea $\left\{L_{j}\right\}$ cualquier sucesión en $\mathcal{B}(\mathcal{H})$ que satisface (6.1). Entonces existe una sucesión de escalares $\left\{c_{j}\right\}$ tales que $\sum_{j}\left(L_{j}-c_{j}\right)^{*}\left(L_{j}-c_{j}\right)$ converge fuertemente.

Demostración: Scan $u, v \in \mathcal{H}$ dos vectores unitarios li. y sean $a_{j}=$ $\left\langle u, L_{j} u\right\rangle, b_{j}=\left\langle v, L_{j} v\right\rangle$. Sea $P$ la proyección ortogonal sobre el subespacio generado por $u$ y $v$, sea $m_{j}=\frac{1}{2} \operatorname{tr} P L_{j} P$. Por la Proposición 8 , ya que $\left\{L_{j}\right\}$ satisface la ecuación $(6.1)$, para toda $P$ proyección ortogonal en $\mathcal{H}$ tenemos que

$$
\sup _{\substack{\|X\| \leq 1 \\\|u\| \leq 1, P u=u}} \sum_{j}\left\|\left[P L_{j} P, P X P\right] u\right\|^{2} \leq \infty .
$$

Observemos que $\left\{P L_{j} P\right\}$ es una sucesión que satisface (6.1) en $\mathcal{B}(\operatorname{span}\{u, v\})$, pues cada operador acotado en span $\{u, v\}$ tiene la loma $X=I^{\prime} X^{\prime} P$ donde $X^{\prime} \in \mathcal{B}(\mathcal{H})$ y además para $w \in \operatorname{span}\{u, v\}$,

$$
\sup _{\substack{\|w\| \leq 1 \\\|X\| \leq 1}} \sum_{j}\left\|\left[P L_{j} P, X\right] w\right\|^{2}=\sup _{\substack{\|u\| \leq 1 \\\left\|X^{\prime}\right\| \leq 1, P u=u}} \sum_{j}\left\|\left[P L_{j} P, I^{\prime} X^{\prime} P\right]\right\|^{2}<\infty
$$

pues

$$
\|X\|_{\mathcal{B}(s p a r t\{u, v\})}=\|X\|=\left\|X^{\prime}\right\| .
$$

Podemos aplicar la Proposición 10 y obtenemos que

$$
\sum_{j} \operatorname{lr}\left(\Gamma L_{j} \Gamma^{\prime}-\frac{1}{2} \operatorname{lr} \Gamma^{\prime} L_{j} P\right)^{*}\left(P L_{j} P-\frac{1}{2} t r l^{\prime} L_{j} l\right)<\infty
$$

$\operatorname{Sea} \Lambda_{j}=P L_{j} P-m_{j} 1$ entonces

$$
\begin{aligned}
& \left\|\Lambda_{j} u\right\|^{2}=\left\langle\Lambda_{j} u, \Lambda_{j} u\right\rangle=\left\langle u, \Lambda_{j}^{*} \Lambda_{j} u\right\rangle \\
& \left\|\Lambda_{j} v\right\|^{2}=\left\langle\Lambda_{j} v, \Lambda_{j} u\right\rangle=\left\langle v, \Lambda_{j}^{*} \Lambda_{j} v\right\rangle
\end{aligned}
$$


$\operatorname{lnogo}$

$$
\left\|\Lambda_{j} u\right\|^{2}+\left\|\Lambda_{j} v\right\|^{2}=\operatorname{tr} \Lambda_{j}^{*} \Lambda_{j}<\infty
$$

por la Proposición 10, es decir

$$
\sum_{j}\left\{\left\|\left(P L_{j} P-m_{j} 1\right) u\right\|^{2}+\left\|\left(P L_{j} P-m_{j} 1\right) v\right\|^{2}\right\}<\infty
$$

Como $P w=w$ para $w$ en $\operatorname{span}\{u, v\}$ y $\|u\|=1$ entonces

$$
\begin{aligned}
\sum_{j}\left\|\left(P L_{j} P-a_{j}\right) u\right\|^{2} & =\sum_{j}\left\|P\left(L_{j}-a_{j}\right) u\right\|^{2} \\
& \leq \sum_{j}\left\|\left(L_{j}-a_{j}\right) u\right\|^{2} \\
& =\sum_{j}\left(\left\|L_{j} u\right\|^{2}-\left|a_{j}\right|^{2}\right)<\infty
\end{aligned}
$$

por la Proposición 7. Similarmente

$$
\sum_{j}\left\|\left(P L_{j} P-b_{j}\right) v\right\|^{2}<\infty
$$

Ahora, por otra parte

$$
\begin{aligned}
m_{j} & =\frac{1}{2} \operatorname{tr}_{\operatorname{span}\{u, v\}} P L_{j} P=\frac{1}{2}\left(\left\langle u, P L_{j} P u\right\rangle+\left\langle v \cdot P L_{j} P v\right\rangle\right) \\
& =\frac{1}{2}\left(a_{j}+b_{j}\right)
\end{aligned}
$$

sustituyendo $m_{j}$ en (11.2) y usando cque $\|u\|=\|v\|=1$ tencmos

$$
\begin{aligned}
\left\|\left(P L_{j} P-m_{j}\right) u\right\|^{2}= & \left\|P L_{j} P u\right\|^{2}-\frac{1}{2}\left(a_{j}+b_{j}\right) \overline{a_{j}} \\
& -\frac{1}{2}\left(\overline{a_{j}}+\overline{b_{j}}\right) a_{j}+\frac{1}{4}\left|a_{j}+b_{j}\right|^{2}
\end{aligned}
$$

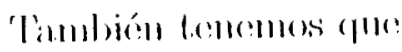

$$
\begin{aligned}
\left\|\left(P L_{j} P-m_{j}\right) v\right\|^{2}= & \left\|P L_{j} P v\right\|^{2}-\frac{1}{2}\left(a_{j}+b_{j}\right) \overline{b_{j}} \\
& -\frac{1}{2}\left(\overline{a_{j}}+\overline{b_{j}}\right) b_{j}+\frac{1}{4}\left|a_{j}+b_{j}\right|^{2}
\end{aligned}
$$

sumando y simplificando (11.4) y (11.5) obtenemos 


$$
\begin{gathered}
\infty>\sum_{j}\left\{\left\|\left(P L_{j} P-m_{j}\right) u\right\|^{2}+\left\|\left(P L_{j} P-m_{j}\right) v\right\|^{2}\right\} \\
=\sum_{j}\left(\left\|P L_{j} P u\right\|^{2}+\left\|P L_{j} P v\right\|^{2}-\frac{1}{2}\left(a_{j} \overline{b_{j}}+\overline{a_{j}} b_{j}\right)-\frac{1}{2}\left|a_{j}\right|^{2}-\frac{1}{2}\left|b_{j}\right|^{2}\right) .
\end{gathered}
$$

Por otra parte,

$$
\begin{aligned}
\left\|\left(P L_{j} P-a_{j}\right) u\right\|^{2} & =\left\langle\left(P L_{j} P-a_{j}\right) u,\left(P L_{j} P-a_{j}\right) u\right\rangle \\
& =\left\|P L_{j} P u\right\|^{2}-\left|a_{j}\right|^{2} .
\end{aligned}
$$

Similarmente,

$$
\left\|\left(P L_{j} P-b_{j}\right) v\right\|^{2}=\left\|P L_{j} P v\right\|^{2}-\left|b_{j}\right|^{2} .
$$

Así cue,

$$
\begin{aligned}
& \sum_{j}\left(\left\|P L_{j} P u\right\|^{2}-\left|a_{j}\right|^{2}\right)<\infty \\
& \sum_{j}\left(\left\|P L_{j} P v\right\|^{2}-\left|b_{j}\right|^{2}\right)<\infty
\end{aligned}
$$

obien

$$
\sum_{j}\left\{\left\|P L_{j} P u\right\|^{2}+\left\|P L_{j} P v\right\|^{2}-\left(\left|a_{j}\right|^{2}+\left|b_{j}\right|^{2}\right)\right\}<\infty
$$

restando a (11.6) la ecuación (11.7) obtenemos

$$
\sum_{j} \frac{1}{2}\left(\left|a_{j}\right|^{2}+\left|b_{j}\right|^{2}-a_{j} \overline{b_{j}}-\overline{a_{j}} b_{j}\right)<\infty
$$

es decir

$$
\sum_{j}\left|\left\langle u, L_{j} u\right\rangle-\left\langle v, L_{j} v\right\rangle\right|^{2}<\infty
$$

Para cualesquiera $u, v$ lineahmente independientes se escoge un vector uni-

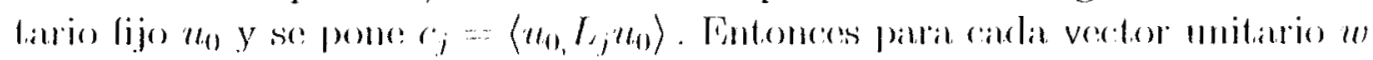
tal (fune w) y $u_{0}$ sem l.i.

$$
\begin{aligned}
\left\|\left(L_{j}-c_{j}\right) w\right\|^{2} & =\left\langle\left(L_{j}-c_{j}\right) w,\left(L_{j}-c_{j}\right) w\right\rangle \\
& =\left\|L_{j} w\right\|^{2}-c_{j} \overline{d_{j}}-\overline{c_{j}} d_{j}+\left|c_{j}\right|^{2}
\end{aligned}
$$


donde $d_{j}=\left\langle w, L_{j} w\right\rangle$ así que

$$
\begin{aligned}
\left|\left\langle w, L_{j} w\right\rangle-\left\langle u_{0}, L_{j} u_{0}\right\rangle\right|^{2} & =\left|d_{j}-c_{j}\right|^{2} \\
& =\left|d_{j}\right|^{2}-c_{j} \overline{d_{j}}-\overline{c_{j}} d_{j}+\left|c_{j}\right|^{2}
\end{aligned}
$$

y

$$
\left\|\left(l_{j} \quad d_{j}\right) w\right\|^{2}-\left\|l_{j} w\right\|^{2} \quad\left|d_{i j}\right|^{2}
$$

entonces

$$
\left\|\left(L_{j}-c_{j}\right) w\right\|^{2}=\left\|\left(L_{j}-d_{j}\right) w\right\|^{2}+\left|d_{j}-c_{j}\right|^{2} .
$$

Por lo tanto

$$
\begin{aligned}
\sum_{j}\left\|\left(L_{j}-c_{j}\right) w\right\|^{2} & =\sum_{j}\left\{\left\|\left(L_{j}-d_{j}\right) w\right\|^{2}+\left|d_{j}-c_{j}\right|^{2}\right\} \\
& =\sum_{j}\left\{\left(\left\|L_{j} u\right\|^{2}-\left|d_{j}\right|^{2}\right)+\left|d_{j}-c_{j}\right|^{2}\right\}<\infty,
\end{aligned}
$$

por la Proposición 7 y por (11.8).

Cuando $w=c u_{0}$ tenemos que

$$
\|\left(L_{j}-c_{j}\right)\left(u_{0} \|^{2}=|c|^{2}\left(\left\|L_{j} u_{0}\right\|^{2}-\left|c_{j}\right|^{2}\right)\right.
$$

y por la proposición 7 obtenemos

$$
\sum_{j}\left\|\left(L_{j}-c_{j}\right) w\right\|^{2}<\infty .
$$

Lo cual innplica convergencia débil pues $\left\langle u, \sum_{j}\left(L_{j}-c_{j}\right)^{*}\left(L_{j}-c_{j}\right) w\right\rangle<\infty$.

Hagamos por simplicidad $L_{j}-c_{j}:=\widetilde{L_{j}}$ y sea $T_{n}=\sum_{j=1}^{n} \widetilde{L}_{j}^{*} \widetilde{L_{j}}$, entonces

$$
T_{1} \leq T_{2} \leq \cdots \leq T_{n} \leq \cdots<\infty
$$

además, $\operatorname{los} T_{n}$ son antoadjuntos para toda $n \in \mathbb{N}$. Por lo tanto ${ }^{13}$, existe $T \in \mathcal{B}(\mathcal{H})$ tal que $T_{n} \stackrel{s}{\rightarrow} T$.

Definición: Para cada operador $X \in \mathcal{B}(\mathcal{H})$ y carla $\varphi \in \mathcal{H}$, defínase el operador $\Phi(X)$ mediante la relación

$$
\text { Ф }(X) \varphi:=\sum_{j} L_{j}^{*} X L_{\lrcorner_{j}} \varphi,
$$

${ }^{13}$ Ver Weidmann $[W]$, teorema 4.28 
donde escribimos $L_{j}$ por $\widehat{L_{j}}$ de la proposición anterior.

Para cada $X \in \mathcal{B}(\mathcal{H})$ tenemos que

$$
\begin{aligned}
|\langle\varphi, \Phi(X) \varphi\rangle| & \leq \sum_{j}\left|\left\langle L_{j} \varphi, X L_{j} \varphi\right\rangle\right| \\
& \leq\|X\| \sum_{j}\left\|L_{j} \varphi\right\|^{2} \\
& =\|X\| \sum_{j}\left\langle\varphi, L_{j}^{*} L_{j} \varphi\right\rangle<\infty
\end{aligned}
$$

para cada $\varphi \in \mathcal{H}$. El principio de acotamiento uniforme aplicado a la sucesión $\sum_{j=1}^{n} I_{j}^{*} L_{j}$ implica que existe mat constante c tal que

$$
\|\Phi(X)\|=\sup _{\|\varphi\| \leq 1}|\langle\varphi, \Phi(X) \varphi\rangle| \leq c\|X\|
$$

es decir, $\Phi(X)$ es un operator acotado para cada $X \in \mathcal{B}(\mathcal{H})$. Entonces $\Phi$ es una transformación de $\mathcal{B}(\mathcal{H})$ en sí mismo, estudiemos sus propiedades.

Primero obsérvese que

$$
\Phi(1)=\sum_{j} L_{j}^{*} L_{j}
$$

y por (D.1) tenemos que

$$
\Phi(X) \leq\|X\| \Phi(1), \text { para todo } X \in \mathcal{B}(\mathcal{H}) .
$$

Además

$$
\langle\varphi, \Phi(1) \varphi\rangle=\sum_{j}\left\langle\varphi, L_{j}^{*} L_{j} \varphi\right\rangle=\sum\left\|L_{j} \varphi\right\|^{2} \geq 0
$$

entonces

$$
\Phi(1) \geq 0
$$

Definición: Sea $I I$ un operador autoarljunto en $\mathcal{B}(\mathcal{H})$ defínase el operador G en $\mathcal{H}$ mediante la relación

$$
G=\frac{1}{2} \Phi(1)-i H .
$$


Tenemos que

$$
\begin{aligned}
\operatorname{Re}\langle\varphi,-G \varphi\rangle & =\left\langle\varphi,-\left(\frac{1}{2} \Phi(1)-i H\right) \varphi\right\rangle+\overline{\left\langle\varphi,-\left(\frac{1}{2} \Phi(1)-i H\right) \varphi\right\rangle} \\
& =-\langle\varphi, \Phi(1) \varphi\rangle \leq 0
\end{aligned}
$$

para cada $\varphi$ en $\mathcal{H}$

Así mismo

$$
\operatorname{Re}\left\langle\varphi,-G^{*} \varphi\right\rangle=-\langle\varphi, \Phi(1) \varphi\rangle \leq 0 .
$$

Entonces como $-G$ y $-G^{*}$ son disipativos, $-G$ es el generador de un semigrupo de contracciones uniformente continuo, pues $G$ es acotado. Denotemos por $u_{t}$ a este semigrupo de contracciones.

Proposición 14: La transformación T es completamente positiva y normal de $\mathcal{B}(\mathcal{H})$ en sí mismo.

Demostración: Inmediata, pues $\Phi(X)$ está en la forma de Kraus (1.5).

Si $\theta$ es el generador del semigrupo dinámico cuántico $T_{\ell}$, entonces para $\operatorname{ardat} X \in \mathcal{B}(\mathcal{H})$

$$
\frac{d}{d t} T_{t}(X)=\theta\left(T_{t}(X)\right), t \geq 0,
$$

donde la derivada es en la norma de $\mathcal{B}(\mathcal{H})$.

Proposición 15: Supóngase que $\theta(X)=-G^{*} X-X G+\Phi(X)$, entonces para cada $X \in \mathcal{B}(\mathcal{H})$ la ecuación diferencial

$$
\frac{d}{d l} l_{1}(X) \varphi=\theta\left(T_{l}(X)\right) \varphi, T_{0}(X)-X
$$

es equivalente a la ecuación integral

$$
T_{\ell}(X) \varphi=w_{l}^{*} X w_{\ell} \varphi+\int_{0}^{\iota} d \tau w_{t-\tau}^{*} \Phi\left(T_{\tau}(X)\right) w_{l-\tau} \varphi
$$

donde la derivada y la integral son en la topología fuerte de $\mathcal{B}(\mathcal{H})$. 
Demostración: $(15.2) \Rightarrow(15.1)$ Sustituyendo $w_{t}=e^{-t G}, w_{t}^{*}=e^{-\iota G^{*}}$ en (15.2) y derivando obtenemos que

$$
\begin{aligned}
\frac{d}{d t} T_{t}(X) \varphi= & -G^{*} w_{t}^{*} X w_{t} \varphi-w_{t}^{*} X w_{t} G \varphi \\
& +\frac{d}{d t} \int_{0}^{t} d \tau w_{t-\tau}^{*} \Phi\left(T_{\tau}(X)\right) w_{t-\tau} \varphi
\end{aligned}
$$

sabemos que

$$
\begin{aligned}
\frac{d}{d t} \int_{0}^{t} d \tau e^{-(l-\tau) G^{*}} \Phi\left(T_{\tau}\right) e^{-(t-\tau) G} \varphi= & \Phi\left(T_{t}(X)\right) \varphi+\int_{0}^{t} d \tau \frac{\partial}{\partial t}\left(w_{t-\tau}^{*} \Phi\left(T_{\tau}(X)\right) w_{\imath-\tau} \varphi\right) \\
= & \Phi\left(T_{t}(X)\right) \varphi+\int_{0}^{t} d \tau\left[-G^{*} w_{t-\tau}^{*} \Phi\left(T_{\tau}(X)\right) w_{t-\tau} \varphi\right]- \\
& -\int_{0}^{t} d \tau\left[-w_{t-\tau}^{*} \Phi\left(T_{\tau}(X)\right) w_{t-\tau} G \varphi\right]
\end{aligned}
$$

entonces

$$
\begin{aligned}
\frac{d}{d t} T_{l}(X) \varphi & =-G^{*} T_{t}(X) \varphi+T_{l}(X) G \varphi+\Phi\left(T_{t}(X)\right) \varphi \\
& =\theta\left(T_{t}(X)\right) \varphi
\end{aligned}
$$

$(15.1) \Rightarrow(15.2)$ Tenemos para $\tau \geq 0$ y para cada $\varphi \in \mathcal{H}$,

$$
\Phi\left(T_{\tau}(X)\right) \varphi=G^{*} T_{\tau}(X) \varphi+T_{\tau}(X) G \varphi-\frac{d}{d \tau} T_{\tau}(X) \varphi \text { y } T_{0}(X)=X .
$$

entonces, para $0 \leq \tau \leq t$ y $\varphi=w_{t-\tau} \psi, \psi \in \mathcal{H}$ obtenemos que

$$
\begin{aligned}
w_{t-\tau}^{*} \Phi\left(T_{\tau}(X)\right) w_{t-\tau} \varphi= & w_{t-\tau}^{*} G^{*} T_{\tau}(X) w_{t-\tau} \varphi+w_{t-\tau}^{*} T_{r}(X) G u_{t-\tau} \varphi \\
& -w_{t-\tau}^{*}\left(\frac{d}{d \tau} T_{\tau}(X)\right) w_{t-\tau} \varphi \\
= & \frac{d}{d \tau} w_{t-\tau}^{*} T_{\tau}(X) w_{t-\tau} \varphi
\end{aligned}
$$

Lemos usado la regla de Leibnitz $\frac{d}{d t} w_{l} \varphi=-G w_{t} \varphi$ y $\left(\frac{d}{d t} w_{t} \varphi\right)^{*}=\frac{d}{d t}\left(w_{t}^{*}\right)$, pues $w_{t}=e^{-t G}$ y $G^{*}$ conmuta con $e^{-t C^{*}}$. Integrando con respecto a $\tau$ en $[0, t]$ se obtiene

$$
\begin{aligned}
\int_{0}^{t} d \tau w_{t-\tau}^{*} \Phi\left(T_{\tau}(X)\right) w_{t-\tau} \varphi & =\left.w_{t-\tau}^{*} T_{\tau}(X) w_{t-\tau} \varphi\right|_{0} ^{l} \\
& =w_{t-\tau}^{*} X w_{t-\tau} \varphi-T_{l}(X) \varphi
\end{aligned}
$$


así

$$
T_{t}(X) \psi=w_{t}^{*} X w_{\iota} \psi+\int_{0}^{t} d \tau w_{t-\tau}^{*} \Phi\left(T_{\tau}(X)\right) w_{t-\tau}
$$

Proposición 16: $\frac{d}{d \tau}\left\|w_{t-\tau} \varphi\right\|^{2}=\left\langle w_{t-\tau} \varphi, \Phi(1) w_{t-\tau} \varphi\right\rangle$, para cada $\varphi \in \mathcal{H}$.

\section{Demostración:}

$$
\begin{aligned}
\frac{d}{d \tau}\left\|w_{t-\tau} \varphi\right\|^{2}= & \frac{d}{d \tau}\left\langle w_{t-\tau} \varphi, w_{t-\tau} \varphi\right\rangle=\left\langle G w_{t-\tau} \varphi, w_{t-\tau} \varphi\right\rangle+\left\langle w_{t-\tau} \varphi, G w_{t-\tau} \varphi\right\rangle \\
= & \left\langle\frac{1}{2} \Phi(1) w_{t-\tau} \varphi, w_{t-\tau} \varphi\right\rangle+i\left\langle\| w_{t-\tau} \varphi, w_{t-\tau} \varphi\right\rangle \\
& +\left\langle w_{t-\tau} \varphi, \frac{1}{2} \Phi(1) w_{t-\tau} \varphi\right\rangle-i\left\langle w_{t-\tau} \varphi, H w_{t-\tau} \varphi\right\rangle
\end{aligned}
$$

observando que tanto $H$ como $\Phi(1)$ son autoadjuntos se cancelan y agrupan términos para obtener lo buscado.

Proposición 17: Sean $T_{l}^{(0)}=0, T_{t}^{(n)}(X)=w_{l}^{*} X w_{l} \psi+\int_{0}^{l} d \tau w_{l-\tau}^{*} \Phi\left(T_{\tau}^{(n-1)}(X)\right) w_{t-\tau} \psi$ entonces $T_{t}^{(n)}(X) \underset{n}{\rightarrow} T_{\text {t }}$ en la topología fucrte de $\mathcal{B}(\mathcal{H})$ y $\widetilde{T}_{t}$ salisface la caunción (15.2).

Demostración: Haremos la demostración siguiendo los siguientes pasos:

(i) Para todo $X \geq 0,0 \leq T_{\ell}^{(n)}(X) \leq\|X\| \cdot 1$

(ii) Para carla $n \in \mathbb{N}$ y carda $X$ en $\overline{\mathcal{B}}(\mathcal{H}), J_{t}^{(n)}(X)$ es uniformemente acotado donde

$$
J_{t}^{(n)}(X)=\int_{0}^{t} d \tau w_{t-\tau}^{*} \Phi\left(T_{\tau}^{(n)}(X)\right) w_{t-\tau}
$$

(iii) Para carla $n \in \mathbb{N}, T_{l}^{(n)}$ satisface la condición (iv) en la definición de semigrupo y es continuo en la norma de $\mathcal{B}(\mathcal{H})$ para cada $t>0$ fijo.

(iv) $T_{\imath}^{(n)}(X) \leq T_{t}^{(n+1)}(X)$ para toda $X \geq 0$.

Sabemos que

$$
\left\langle\varphi, T_{l}^{(n)}(X) \varphi\right\rangle=\left\langle u_{\ell} \varphi, X w_{l} \varphi\right\rangle+\int_{0}^{t} d \tau\left\langle w_{l-\tau} \varphi, \Phi\left(T_{\tau}^{(n-1)}(X)\right) w_{\ell-\tau} \varphi\right\rangle .
$$

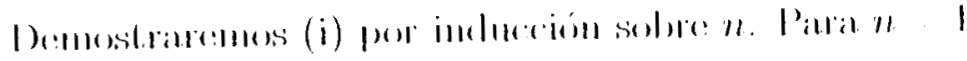

$$
\begin{aligned}
& 0 \leq\left\langle\varphi, T_{1}^{(1)}(X) \varphi\right\rangle=\left\langle w_{1} \varphi, X w_{\imath} \varphi\right\rangle \\
& \leq\left\|w_{l} \varphi\right\|\left\|X w_{l} \varphi\right\| \leq\|. X\|
\end{aligned}
$$


pules $w_{l}$ es de contracciones $y X$ es positivo. Entonces $0 \leq T_{l}^{(1)}(X) \leq$ $\|X\| \cdot 1$. Supongamos $0 \leq T_{t}^{(n-1)}(X) \leq\|X\| \cdot 1$ para cada $X \geq 0$, entonces $\int_{0}^{l} d \tau\left\langle w_{t-\tau} \varphi, \Phi\left(T_{\tau}^{(n-1)}(X)\right) w_{t-\tau} \varphi\right\rangle \geq 0$, pues $\Phi$ es completamente positivo $\mathrm{y}$

$$
\begin{aligned}
0 & \leq\left|\left\langle\varphi, T_{t}^{(n)}(X) \varphi\right\rangle\right| \\
& \leq\left\|w_{t} \varphi\right\|^{2}\|X\|+\left|\int_{0}^{t} d \tau\left\langle w_{t-\tau} \varphi, \Phi\left(T_{\tau}^{(n \cdots 1)}(X)\right) w_{t-\tau} \varphi\right\rangle\right|
\end{aligned}
$$

pero

$\left|\int_{0}^{t} d \tau\left\langle w_{t-\tau} \varphi, \Phi\left(T_{\tau}^{(n-1)}(X)\right) u_{t-\tau} \varphi\right\rangle\right| \leq \int_{0}^{t} d \tau\left\|T_{\tau}^{(n-1)}(X)\right\|\left\langle u_{t-\tau} \varphi, \Phi(1) w_{t-\tau} \varphi\right\rangle$

pues $\langle\varphi, \Phi(X) \varphi\rangle \leq\|X\|\langle\varphi, \Phi(1) \varphi\rangle$. Además por la Proposición 16 y la hipótesis de inducción, la expresión del lado derecho de (17.1) es menor igual que

$$
\|X\| \int_{0}^{l} d \tau \frac{d}{d \tau}\left\|w_{t-\tau} \varphi\right\|^{2}=\|X\|\left(\|\varphi\|^{2}-\left\|w_{t-\tau} \varphi\right\|^{2}\right)
$$

entonces

$$
\begin{aligned}
0 & \leq\left\langle\varphi, T_{\tau}^{(n)}(X) \varphi\right\rangle \\
& \leq\|X\|\left\|w_{t-\tau} \varphi\right\|^{2}+\|X\|\left(\|\varphi\|^{2}-\left\|w_{t-\tau} \varphi\right\|^{2}\right) \\
& =\|X\|\|\varphi\|^{2},
\end{aligned}
$$

es decir se cumple (i).

La propiedad (ii) se sigue de la desigualdad (17.1) y la desigualdarl anterior.

Probarenos (iii) y (iv) simultáneamente por inducción:

$T_{\tau}^{(1)}(X)=w_{t}^{*} X w_{t}$ entonces para cadd $\varphi \in \mathcal{H}$ y carla par de sucesionces finitas $\left(X_{i}\right),\left(Y_{i}\right)$ cn $\mathcal{B}(\mathcal{H})$ tenemos que

$$
\begin{aligned}
\left\langle\sum_{i, j} Y_{i} \varphi, T_{r}^{(1)}\left(X_{i}^{*} X_{j}\right) Y_{j \varphi}^{\prime}\right\rangle & =\sum_{i, j}\left\langle Y_{i} \varphi, w_{l}^{*}\left(X_{i}^{*} X_{j}\right) w_{l} Y_{j} \varphi\right\rangle \\
& =\left\langle\sum_{i} X_{i} w_{l} Y_{i} \varphi, \sum_{i} X_{i} w_{t} Y_{i} \varphi\right\rangle \\
& =\left\|\sum_{i} X_{i} w_{t} Y_{i} \varphi\right\|^{2} \\
& \geq 0
\end{aligned}
$$


por otra parte

$$
0=T_{i}^{(0)} \leq T_{t}^{(1)}
$$

Para $n=1, T_{t}^{(1)}=w_{t}^{*} X w_{t}$. Si $X_{n} \rightarrow X$ en la norma, es decir, si $\left\|X_{n}-X\right\| \underset{n}{\rightarrow}$ 0 entonces

$$
\begin{aligned}
\left\|T_{t}^{(1)}\left(X_{n}\right)-T_{t}^{(1)}(X)\right\| & =\sup _{\|\varphi\|=1}\left\|T_{t}^{(1)}\left(X_{n}\right) \varphi-T_{t}^{(1)}(X) \varphi\right\| \\
& =\sup _{\|\varphi\|=1}\left\|w_{t}^{*} X_{n} w_{\imath} \varphi-w_{t}^{*} X w_{t} \varphi\right\| \\
& \leq \sup _{\|\varphi\|=1}\left\|X_{n}-X\right\|\left\|w_{t} \varphi\right\| \\
& \leq\left\|X_{n}-X\right\| \underset{n}{\rightarrow} 0
\end{aligned}
$$

es decir $T_{t}^{(1)}$ es continuo en la norma de $\mathcal{B}(\mathcal{H})$.

Supongamos que (iii) se cumple para $n$. Escribimos

$$
T_{t}^{(n+1)}(X)=T_{t}^{(1)}(X)+\int_{0}^{t} d \tau T_{t-\tau}^{(1)}\left(\Phi\left(T_{\tau}^{(n)}(X)\right)\right)
$$

como $T_{(\cdot)}^{(1)}$, y y $T_{l}^{(n)}$ cumplen la propiedad (iv) en la definición de stle, por hipótesis, y dada la linealidad de la integral, $T_{t}^{(n+1)}$ también satisface la propiedad (iv).

Supongamos que $X_{N} \underset{N}{\rightarrow} X$ en la norma de operadores entonces

$$
\begin{aligned}
\left\|T_{t}^{(n)}\left(X_{N}\right)-T_{t}^{(n)}(X)\right\| & =\left\|T_{l}^{(1)}\left(X_{N}-X\right)+\int_{0}^{t} d \tau T_{t-\tau}^{(1)}\left(\Phi\left(T_{r}^{(n)}\left(X_{N}-X\right)\right)\right)\right\| \\
& \leq\left\|X_{N}-X\right\|+\int_{0}^{t} d \tau\left\|T_{t-\tau}^{(1)}\left(\Phi\left(T_{\tau}^{(n)}\left(X_{N}-X\right)\right)\right)\right\| \\
& \leq\left\|X_{N}-X\right\|+\int_{0}^{t} d \tau\left\|\Phi\left(T_{\tau}^{(n)}\left(X_{N}-X\right)\right)\right\| \\
& \leq\left\|X_{N}-X\right\|+\int_{0}^{t} d \tau\left\|T_{\tau}^{(n)}\left(X_{N}-X\right)\right\|\|\Phi(1)\| \\
& \leq\left\|X_{N}-X\right\|+\beta t\left\|X_{N}-X\right\| \rightarrow{ }_{N} 0,
\end{aligned}
$$

$\operatorname{con} \beta=\|\Phi(1)\| \mathrm{y} X \geq 0$.

Pues

$$
\begin{aligned}
\|\Phi(X)\| & =\sup _{\|\varphi\|=1}\langle\varphi, \Phi(X) \varphi\rangle \\
& \leq\|X\| \sup _{\|\varphi\|=1}\langle\varphi, \Phi(1) \varphi\rangle \\
& =\|X\|\|\Phi(1)\|
\end{aligned}
$$


ya que $\Phi(X)$ es positivo y autoadjunto, $\Phi(1)$ es autoadjunto y acotado y, arlemás, $T_{\imath}^{(n)} \leq\|X\| \cdot 1$, por hipótesis de inducción. Si $X$ no es positivo se escribe como combinación lineal de cuatro operadores positivos y

$$
\begin{aligned}
\|\Phi(X)\| & =\left\|\sum_{i=1}^{4} \Phi\left(c_{i} X_{i}\right)\right\| \\
& \leq \sum_{i=1}^{4}\left|c_{i}\right|\left\|\Phi\left(X_{i}\right)\right\| \\
& \leq \sum_{i=1}^{4}\left|c_{i}\right|\left\|X_{i}\right\|\|\Phi(1)\| \\
& \leq 4 k\|X\|
\end{aligned}
$$

donde $k=$ máx $\left(\left|c_{i}\right|\right)$. Con esto terminamos la demostración de (iii).

Para la propiedad (iv) escribimos

$$
\begin{aligned}
T_{t}^{(n+1)}(X)-T_{t}^{(n)}(X) & =\int_{0}^{t} d \tau\left\{T_{t-\tau}^{(1)}\left(\Phi\left(T_{\tau}^{(n)}(X)\right)\right)-T_{t-\tau}^{(1)}\left(\Phi\left(T_{\tau}^{(n-1)}(X)\right)\right)\right\} \\
& =\int_{0}^{t} d \tau\left\{T_{t-\tau}^{(1)}\left(\Phi\left(T_{\tau}^{(n)}(X)-T_{\tau}^{(n-1)}(X)\right)\right)\right\} \\
& \geq 0
\end{aligned}
$$

pues por hipótesis de inducción $T_{t}^{(n)}(X)-T_{t}^{(n-1)}(X) \geq 0$ y $\Phi(X) \geq 0$ si $X \geq 0$.

Por las propiedades (i) a (iv) tenemos que $\left\{\left\langle\varphi, T_{\imath}^{(n)}(X) \varphi\right\rangle\right\}_{n \geq 1}$ es una sucesión creciente de números reales para cada $\varphi \in \mathcal{H}$ y $X \in \mathcal{B}(\mathcal{H}), X \geq 0$. Sea

$$
\widetilde{T_{t}}(X)[\varphi]:=\sup _{n}\left\langle\varphi, T_{t}^{(n)}(X) \varphi\right\rangle, X \geq 0 .
$$

$\widetilde{T}_{t}(X)[\varphi]$ es una forma cuadrática acotada, pues

$$
0 \leq\left\langle\varphi, T_{t}^{(n)}(X) \varphi\right\rangle \leq\|X\|\|\varphi\|^{2}, \forall n
$$

para cada $X \geq 0$. Entonces por el teorema de representación de formas cuadráticas $^{14}$, existe $\widetilde{T}_{l}(X)$ en $\mathcal{B}(\mathcal{H})$ tal que $\widetilde{T}_{l}(X)[\varphi]=\widetilde{T}_{l} \varphi$. Además $T_{t}^{(n)}(X) \nearrow^{w} \widehat{T}_{t}(X), X \geq 0$. Tenemos que para $X \geq 0, T_{t-\tau}^{(1)}\left(\Phi\left(T_{r}^{(n)}(X)\right)\right) \nearrow^{w}$

\footnotetext{
${ }^{14}$ Ver Káto $[K a]$, capítulo VI, teorena 2.1
} 
$T_{t-\tau}^{(1)}\left(\Phi\left(T_{\tau}(X)\right)\right)$ y la convergencia también es en sentido fuerte pues la sucesión es creciente. Así mismo, por el teorema de convergencia monótona.

$$
\int_{0}^{t} d \tau T_{t-\tau}^{(1)}\left(\Phi\left(T_{\tau}^{(n)}(X)\right)\right)^{w} \int_{0}^{t} d \tau T_{t-\tau}^{(1)}\left(\Phi\left(\widetilde{T_{\tau}}(X)\right)\right)
$$

y la convergencia también es fuerte. Por lo tanto, $\widetilde{T}_{t}$ satisface la misma ecuación (15.2). Para $X$ general basta representar a este operador como combinación lineal de operadores positivos.

Teorema :(Gorini-Kossakowski-Sudarshan, Lindblad) Sea $\mathcal{H}$ un espacio de Hilbert. Un operador $\theta$ en $\mathcal{B}(\mathcal{H})$ es el generador de un semigrupo dinámico cuántico uniformemente continuo si y sólo si, existen un espacio de Hilbert $\mathcal{K}$, un operador acotado $L: \mathcal{H} \rightarrow \mathcal{H} \otimes \mathcal{K}$ y un operador acotado y autoadjunto $I I$ en $\mathcal{H}$ tales que se satisface lo siguiente:

(i) $\theta(X)=i[H, X]-\frac{1}{2}\left\{L^{*} L X+X L^{*} L-2 L^{*} X \otimes 1 L\right\}$ para todo $X \in \mathcal{B}(\mathcal{H})$.

(ii) El conjunto

$$
\{(L X-(X \otimes 1) L) u \mid X \in \mathcal{B}(\mathcal{H}), u \in \mathcal{H}\}
$$

es total en $\mathcal{H} \otimes \mathcal{K}$

Demostración: $(\Rightarrow)$ Sea $\theta$ generador del s.d.c. $\left\{T_{t}\right\}$ en $\mathcal{B}(\mathcal{H})$. Por las Proposicion 2 y el Corolario 1 podemos construir un espacio de Hilbert $\mathcal{K}$ y un mapeo lineal $\pi: \mathcal{B}(\mathcal{H}) \rightarrow \mathcal{B}(\mathcal{H} \otimes \mathcal{K}$ ) tal que se cumplen las propiedades (i) a (iii) del Corolario 1 y el conjunto $\{\pi(X) u \mid X \in \mathcal{B}(\mathcal{H}), u \in \mathcal{H}\}$ es total en $\mathcal{H} \otimes \mathcal{K}$. Como en la prueba de la Proposición 6 construimos el isomorfismo unitario $\Gamma_{0}: \mathcal{H} \otimes \mathcal{K} \rightarrow \mathcal{H} \oplus \mathcal{H} \oplus \cdots$ tal que $\Gamma_{0} \pi(X) u=\oplus_{j}\left[\widetilde{L_{j}}, X\right] u$ para todo $X \in \mathcal{B}(\mathcal{H}), u \in \mathcal{H}$ y la ecuación (6.1) se cumple. Por la Proposición 11 escogemos $\left\{c_{j}\right\}$ tal que: $\sum_{j}\left\|\widetilde{L_{j}}-c_{j}\right\|^{2}<\infty$. Sea $L_{j}=\widetilde{L_{j}}-c_{j}$ entonces $\sum_{j}\left\|L_{j} u\right\|^{2}<\infty$, y $\sum_{j} L_{j}^{*} L_{j}$ es fuertemente convergente. Definimos

$$
L u=\Gamma_{0}^{-1}\left\{\bigoplus_{j} L_{j} u\right\}
$$

entonces

$$
\pi(X)=L X-X \otimes 1_{\mathcal{K}} L, X \in \mathcal{B}(\mathcal{H})
$$

donde $1_{\mathcal{K}}$ es el operarlor identidad en $\mathcal{K}$. Efectivamente,

$$
\pi(X) u=\Gamma_{0}^{-1}\left\{\underset{j}{\oplus}\left[L_{j}, X\right] u\right\}
$$


como en la prueba de la Proposición 6

$$
\begin{aligned}
& =\Gamma_{0}^{-1}\left\{\underset{j}{\oplus}\left(L_{j} X-X L_{j}\right) u\right\} \\
& =\Gamma_{0}^{-1}\left(\underset{j}{\oplus} L_{j} X u\right)-\Gamma_{0}^{-1}\left(\underset{j}{\oplus} X L_{j} u\right) \\
& =L X u-\Gamma_{0}^{-1}\left(X \underset{j}{\oplus} L_{j} u\right)
\end{aligned}
$$

Sea

$$
\theta_{1}(X)=-\frac{1}{2}\left\{L^{*} L X+X L^{*} L-2 L^{*} X \otimes 1 L\right\}
$$

usando la propiedad (ii) del Corolario 1 podemos demostrar que

$$
\begin{aligned}
\theta_{1}\left(X^{*} Y\right)-\theta_{1}\left(X^{*}\right) Y-X \theta_{1}(Y) & =\pi(X)^{*} \pi(Y) \\
& =\theta\left(X^{*} Y\right)-\theta\left(X^{*}\right) Y-X \theta(Y)
\end{aligned}
$$

para todo $X, Y \in \mathcal{B}(\mathcal{H})$.

Tenemos

$$
\begin{aligned}
\theta_{1}\left(X^{*} Y\right) & =-\frac{1}{2}\left\{L^{*} L X^{*} Y+X^{*} Y L^{*} L-2 L^{*} X^{*} Y \otimes 1 L\right\} \\
\theta_{1}\left(X^{*}\right) Y & =\frac{1}{2}\left\{L^{*} L X^{*} Y+X^{*} L^{*} L Y-2 L^{*} X^{*} \otimes 1 L Y\right\} \\
-X^{*} \theta_{1}(Y) & =\frac{1}{2}\left\{X^{*} L^{*} L Y+X^{*} Y L^{*} L-2 X^{*} L^{*} Y \otimes 1 L\right\}
\end{aligned}
$$

entonces

$$
\begin{aligned}
\theta_{1}\left(X^{*} Y\right)-\theta_{1}\left(X^{*}\right) Y-X \theta_{1}(Y)= & X^{*} L^{*} L Y+L^{*} X^{*} Y \otimes 1 L- \\
& -L^{*} X^{*} \otimes 1 L Y-X^{*} L^{*} Y \otimes 1 L
\end{aligned}
$$

Por otra parte

$$
\begin{aligned}
\pi(X)^{*} \pi(Y)= & \left((L X)^{*}-(X \otimes 1 L)^{*}\right)(L Y-Y \otimes 1 L) \\
= & \left(X^{*} L^{*}-L^{*} X^{*} \otimes 1\right)(L Y-Y \otimes 1 L) \\
= & X^{*} L^{*} L Y-X^{*} L^{*} Y \otimes 1 L- \\
& -L^{*} X^{*} \otimes 1 L Y+L^{*} X^{*} \otimes 1 Y \otimes 1 L
\end{aligned}
$$


pero

$$
\left(X^{*} \otimes 1\right)(Y \otimes 1)=X^{*} Y \otimes 1
$$

de donde se sigue la igualdad $(* *)$ y así $\theta-\theta_{1}$ es una derivación acotada en $\mathcal{B}(\mathcal{H})$. Más aún, tenemos $\theta(X)^{*}=\theta\left(X^{*}\right)$ y $\theta_{1}(X)^{*}=\theta_{1}\left(X^{*}\right)$ puess

$$
\theta_{1}\left(X^{*}\right)=--\frac{1}{2}\left\{L^{*} L X^{*}+X^{*} L^{*} L-2 L^{*} X^{*} \otimes 1 L\right\}
$$

y

$$
\theta_{1}(X)^{*}=-\frac{1}{2}\left\{\left(L^{*} L X\right)^{*}+\left(X L^{*} L\right)^{*}-2\left(L^{*} X \otimes 1 L\right)^{*}\right\}
$$

de donde se signe farihnente la igualdad buscada. Por lo tanto $\theta(X)-$ $\theta_{1}(X)=i[H, X]$ para toda $X$ y algún $H$ acotado y autoadjunto.

Finalmente, queremos que $L$ sea acotado, pero

$$
\begin{aligned}
L u & =\Gamma_{0}^{-1}\left\{\underset{j}{\oplus} L_{j} u\right\} \\
\|L u\|^{2} & \leq\left\|\Gamma_{0}^{-1}\right\| \sum_{j}\left\|L_{j}\right\|^{2}=\sum_{j}\left\|L_{j}\right\|^{2}<\infty
\end{aligned}
$$

punes $\Gamma_{0}^{-1}$ es mitario.

$(\Leftrightarrow) \theta$ es acotado ya que $L, X, H$ son acotados y $\|X \otimes 1\|=\|X\|\|1\|=$ $\|X\|$. Sea

$$
T_{t}=e^{t 0}
$$

este es un semigrupo uniformemente continuo en $\mathcal{B}(\mathcal{H})$. En efecto, $T_{0}=1 \mathrm{y}$ calculando directamente de la serie obtenemos $T_{t+s}=T_{t} T_{s}$ arlemás, tenemos la estimación

$$
\left\|T_{t}-1\right\|<t\|\theta\| e^{t\|\theta\|}
$$

y

$$
\left\|\frac{T_{t}-1}{t}-\theta\right\| \leq\|\theta\|\left\|T_{t}-1\right\|
$$

lo que implica que $\left\{T_{t}\right\}$ es semigrupo uniformemente continuo de operadores acotados con generador $\theta$. Además, darlo que

$$
T_{l}(1)=1+\frac{t \theta(1)}{1 !}+\frac{t^{2} \theta^{2}(1)}{2 !}+\cdots
$$


y $\theta(1)=0$ obtenemos $T_{t}(1)=1$.

Considérese el homorfismo unitario $\Gamma_{0}: \mathcal{H} \otimes \mathcal{K} \rightarrow \mathcal{H} \oplus \mathcal{H} \oplus \cdots$ (pue satisface $\Gamma_{0} u \otimes v=\oplus_{j}\left\langle e_{j}, v\right\rangle u$ donde $e_{1}, e_{2}, \ldots$ es una base ortonormal en $\mathcal{K}$. Entonces $L$ esta dado por $\Gamma_{0} L u=\bigoplus_{j} L_{j} u$ para lodo $n$ en $\mathcal{H}$, donde $\left\{L_{j}\right\}$ es una sucesión de operadores acotados en $\mathcal{H}, \sum_{j} L_{j}^{*} L_{j}$ converge fuertemente y $\|L\|^{2}=\sum_{j}\left\|L_{j}\right\|^{2}$ la propiedad (i) se puede escribir como

$$
\theta(X)=i[H, X]-\frac{1}{2} \sum_{j}\left(L_{j}^{*} L_{j} X+X L_{j}^{*} L_{j}-2 L_{j}^{*} X L_{j}\right)
$$

También como

$$
\begin{aligned}
\theta(X) & =\Phi(X)-\left(\frac{1}{2} \Phi(1)-i H\right) X-X\left(\frac{1}{2} \Phi(1)+i H\right) \\
& =\Phi(X)-G X-X G^{*}
\end{aligned}
$$

donde

$$
G=\frac{1}{2} \Phi(1)-i H
$$

Sea $\widetilde{T}_{\imath}$ la solución de (15.2) construida en la Proposición 17. Ja proposición 15 y el teorema de unicidad para ecuaciones diferenciales implican que $T_{t}=$ $T_{t}$. Entonces $T_{t}$ es un semigrupo en $\mathcal{B}(\mathcal{H})$ uniformemente continuo que cumple la propiedad (iv) en la definicion de sdc. Notemos que $T_{t}$ es de contracciones pues

$$
\left\|T_{i}^{(n)}(X)\right\| \leq\|X\|
$$

para toda $n$ y por lo tanto lo mismo es cierto para $T_{t}$.

Por (13.1) y (13.2), $-G$ es generador de un semigrupo de contracciones uniformemente continuo en $\mathcal{H}, w_{t}$. Mostraremos ahora que $T_{t}$ es normal. Si $X_{N} \stackrel{w}{\rightarrow} X$ (podemos suponer que $X_{N} \stackrel{w^{\prime}}{\nearrow} X$ ), tenemos para todo $t \geq 0$ fijo que

$$
\left\langle w_{t} \varphi, w_{t} X_{N} \psi\right\rangle \underset{N}{\rightarrow}\left\langle w_{t} \varphi, w_{t} X \psi\right\rangle
$$

para todo $\varphi, \psi$ en $\mathcal{H}$. Entonces $T_{t}^{(1)}$ es normal.

Supóngase que $T_{t}^{(n)}$ es normal y recordemos que $\Phi$ también es normal, entonces como la composición de transformaciones normales es ma transformación normal

$$
T_{l-r}^{(1)}\left(\boldsymbol{D}\left(T_{r}^{(n)}\left(X_{N}\right)\right)\right) /_{N}^{w} T_{t}^{(1)}\left(\Phi\left(T_{r}^{(n)}(X)\right)\right),
$$


pues todas estas transformaciones preservan positividad. De aquí resulta por el teorema de convergencia monótona que

$$
\begin{aligned}
\left\langle\varphi, \int_{0}^{t} d \tau T_{t-\tau}^{(1)}\left(\Phi\left(T_{\tau}^{(n)}\left(X_{N}\right)\right)\right) \psi\right\rangle= & \int_{0}^{t} d \tau\left\langle\varphi, T_{t-\tau}^{(1)}\left(\Phi\left(T_{\tau}^{(n)}\left(X_{N}\right)\right)\right) \psi\right\rangle \\
& -\vec{N} \int_{0}^{t} d \tau\left\langle\varphi, T_{t-\tau}^{(1)}\left(\Phi\left(T_{\tau}^{(n)}(X)\right)\right) \psi\right\rangle \\
= & \left\langle\varphi, \int_{0}^{t} d \tau T_{t-\tau}^{(1)}\left(\Phi\left(T_{\tau}^{(n)}(X)\right)\right) \psi\right\rangle
\end{aligned}
$$

y de esto se sigue que $T_{t}$ es normal. 


\section{Apendice 1}

\section{Teorema de \\ Gelfand-Naimark-Segal}

Sea $V$ un conjunto arbitrario y sea $K: V \times V \rightarrow \mathbb{C}$ una función que satisface lo siguiente:

$$
\sum_{i, j} \overline{\alpha_{i}} r_{j} K\left(x_{i}, x_{j}\right) \geq 0
$$

para toda $\alpha_{i} \in \mathbb{C}, x_{i} \in V, i=1, \ldots, n$. A tal función se le llama kernel definido positivo en $V$.

La construcción de Gelfand-Naimark-Segal (GNS) permite asociar con $(V, K)$ otro par $(\mathcal{H}, \pi)$, único salvo isomorfismo, donde $\mathcal{H}$ es un espacio de Hilbert y $\pi$ es una función tal que

$$
K(x, y)=\langle\pi(x), \pi(y)\rangle_{H} .
$$

A $(\mathcal{H}, \pi)$ se le llama par de Gelfand asociado a $(V, K)$.

Tenemos entonces el siguiente

Teorema: (Construcción de Gelfand-Naimark-Segal) Sea $W$ cualquier conjunto y sea $K$ un kernel definido positivo en $W$. Entonces existen un espacio de IItlbert $\mathcal{H}$ y una función $\pi: W \rightarrow \mathcal{H}$ que satisface

(i) El comjunto $\{\pi(x), x \in \mathbb{W}\}$ es tolal en $\mathcal{H}$.

(ii) $K(x, y)=\langle\pi(x), \pi(y)\rangle$ para todo $x, y \in W$.

(iii) Si $\mathcal{H}^{\prime}$ cs otro espacio de Itilbert $y \pi^{\prime}: \mathcal{H} \rightarrow \mathcal{H}^{\prime}$ es otra transformación que satisface (i) y (ii) con $\mathcal{H} y \pi$ reemplazadas por $\mathcal{H}^{\prime}$ y $\pi^{\prime}$ respectivamente, entonces existe un isomorfismo unitario $U: \mathcal{H} \rightarrow \mathcal{H}^{\prime}$ tal que $U \pi(x)=\pi^{\prime}(x)$ para toda $x \in \mathcal{W}$. 
Demostración: Tomemos el conjunto $\mathbb{C}^{W}=\{f: W \rightarrow \mathbb{C}\}$ considérense las funciones $\chi_{\{x\}}: W \rightarrow \mathbb{C}$ definidas por

$$
\chi_{\{x\}}(y)=\left\{\begin{array}{l}
0 \text { si } x \neq y \\
1 \text { si } x=y
\end{array}\right.
$$

Sea $V=\operatorname{span}\left\{\chi_{\{x\}}: x \in W\right\}$, el conjunto $\left\{\chi_{\{x\}}: x \in W\right\}$ es l.i. en $V$ pues si $x_{1}, x_{2} \ldots \in W, \alpha_{1}, \alpha_{2}, \ldots \in \mathbb{C}$ entonces, si $\sum_{i} \alpha_{i} \chi_{\left\{x_{i}\right\}}\left(x_{j}\right)=0$, tenemos que

$$
\alpha_{j}=\sum_{i} \alpha_{i} \chi_{\left\{x_{i}\right\}}\left(x_{j}\right)=0
$$

para cada $j$. Definimos ahora

$$
\left\langle\sum_{i} \alpha_{i} \chi\left\{x_{i}\right\}, \sum_{j} \alpha_{j} \chi_{\left\{x_{j}\right\}}\right\rangle_{V}:=\sum_{i, j} \overline{\alpha_{i}} \alpha_{j} K\left(x_{i}, x_{j}\right)
$$

Sea $N=\left\{x \in W:\langle\chi(x), \chi(x)\rangle_{V}=0\right\}$ entonces la completación de $V / N$ es el espacio de Hilbert $\mathcal{H}$ buscado.

$\mathrm{Si}$ definimos $\pi: W \rightarrow V / N$ por la correspondencia $\pi(x)=\overline{\chi\{x\}}$ donde $\overline{\chi\{x\}}$ es la clase de equivalencia de $\chi_{\{x\}}(y)$ tenemos el par de Gelfand $(V / N, \pi)$ buscado.

Para probar (iii) considérese $S=\{\pi(x): x \in W\}, S^{\prime}=\{\pi(x): x \in W\}$ y la transformación $U: \pi(x) \rightarrow \pi^{\prime}(x)$ de $S$ en $S^{\prime}$. Entonces $U$ es una transformación que preserva el producto escalar, por lo tanto ${ }^{15}$, se extiende de manera única a un isomorfismo de $\mathcal{H}$ en $\mathcal{H}^{\prime}$.

Para una demostración de este teorema aplicado a formas, ver V.S. Sunder $[S]$.

\footnotetext{
${ }^{15}$ Ver Pathasarathy $[P 2]$, Proposición7.2
} 


\section{Apendice 2}

\section{Teorema de Stone-von Neumann.}

Definición: Sea $U_{t}$ un grupo unitario fuertemente continuo en un espacio de Hilbert $\mathcal{H}$. Un subespacio cerrado $D_{-} \subset \mathcal{H}$ se llama entrante si:

(i) $U_{l}\left[D_{\ldots}\right] \subset D_{\ldots}$ para $t \leq 0$.

(ii) $\cap_{t} U_{t}\left[D_{-}\right]=\{0\}$

(iii) $\overline{U_{l}\left[D_{-}\right]}=\mathcal{H}$

Teorema: Sean $U_{t}, V_{t}$ dos grupos uniparamétricos fuertemente continuos en un espacio de Hibert $\mathcal{H}$ que satisfacen

$$
U_{t} V_{s}=e^{i t s} V_{s} U_{t} \text { para todo } s, t
$$

Entonces existe un espacio de Hilbert $\mathcal{N}$ y un operador unitario $\Gamma: \mathcal{H} \rightarrow$ $\mathcal{L}^{2}(\mathbb{R} ; \mathcal{N})$ tal que $\left(\Gamma U_{t} \Gamma^{-1} f\right)(x)=f(x-t) y\left(\Gamma V_{s} \Gamma^{-1} f\right)(x)=e^{-i s x} f(x)$.

Demostración: Sean $P, Q$, autoadjuntos con $U_{t}=e^{-i t P}$ y $V_{s}=e^{-i s Q}$. Sea $\mathcal{D}$ el conjunto de vectores en $\mathcal{H}$ de la forma

$$
\varphi_{f}=\int_{-\infty}^{\infty} \int_{-\infty}^{\infty} f(t, s) U_{t} V_{s} \varphi d t d s
$$

donde $\varphi \in \mathcal{H}$ y $f \in C_{0}^{\infty}\left(\mathbb{R}^{2}\right)$. Se puede probar ${ }^{16}$ que $\mathcal{D}$ es denso en $\mathcal{H}$, $\mathcal{D} \subset D(Q), \mathcal{D} \subset D(P)$, y que $\mathcal{D}$ es invariante bajo $U_{s}, V_{\iota}$. Por el teorema

${ }^{16}$ Simon y Reed $[S-R]$, teorema VIII.8. 
de Stone $P$ y $Q$ son esencialmente autoadjuntos en $\mathcal{D}$. Scal $\psi \in \mathcal{D}$, como $U_{t} \psi$ está también en $\mathcal{D}$, podemos derivar ambos miembros de la igualdad

$$
U_{t} V_{s}=e^{i t s} V_{s} U_{t}
$$

con respecto a $s$. Poniendo $s=0$, obtenemos

$$
U_{i} Q U_{-t} \psi=(Q-t i) \psi
$$

Dado que esta relación es cierta en $\mathcal{D}$, el cual es un esencia de $Q$ y de $Q-t I$, concluimos que $Q$ y $Q-t I$ son unitariamente equivalentes y A2.1 se cumple para todo $\psi \in D(Q)$. Aliora, sea $\left\{E_{\Omega}\right\}$ la familia espectral de $Q$. Entonces $\left\{U_{t} E_{\Omega} U_{-t}\right\}$ es una familia espectral para $U_{t} Q U_{-t}$. Dado que $E_{\Omega}=\chi_{\Omega}(Q),(A 2.1)$ implica que

$$
U_{t} E_{(-\infty, x)} U_{-t}=E_{(-\infty, x+t)}
$$

para todo $x$ y $t$ en $\mathbb{R}$.

Sea $D_{-}=\operatorname{Ran} E_{(-\infty, 0]}$. Mostraremos que $D_{-}$es un subespacio entrunte para $U_{l}$ en $\mathcal{H}$. Primeramente, $(A 2.2)$ implica que $U_{l} D_{-}=\operatorname{Ran} E_{(-\infty, x+\ell]}$ bara todo t. Así,

(i) $U_{l} D_{-} \subset D_{-}, t \leq 0$

(ii) $\cap_{i} U_{t} D_{-}=\{0\}$;

(iii) $\overline{U_{t} U_{t} D_{-}}=\mathcal{H}$

por las propiedades usuales de las proyecciones. Tenemos así ${ }^{17}$, que existe un espacio de Hilbert auxiliar $\mathcal{N}$ y un operador unitario $\Gamma_{-}$de $\mathcal{H}$ en $\mathcal{L}^{2}(\mathbb{R} ; \mathcal{N})$ tal que $\Gamma_{-} D_{-}=\mathcal{L}^{2}(-\infty, 0 ; \mathcal{N})$ y $\Gamma_{-} U_{t} \Gamma_{-}^{-1}$ es una traslación a la derecha en $t$ unidades. Finalmente, dado que $\Gamma_{-} E_{(-\infty, 0)}^{-} \Gamma_{-}^{-1} \chi_{(-\infty, 0)},(A 2.2)$ implica que $\Gamma_{-} E_{(-\infty, x)} \Gamma_{-}^{-1}=\chi_{(-\infty, x)}$ para toda $x$. Por tanto, $\Gamma_{-} Q \Gamma_{-}^{-1}$ es una multiplicación por $x$ y $\Gamma_{-} e^{-i Q s} \Gamma_{-}^{-1}$ es igual a una multiplicación por $e^{-i s x}$.

\footnotetext{
${ }^{17}$ Simon y Reed op. cit. teorema XI.82.
} 


\section{Apendice 3}

\section{Teorema de representación de derivaciones de álgebras de von Neumann.}

En este apéndice, se da una caracterización para los operadores derivación que se utiliza ampliamente en la demostración del teorema de principal de esta tesis. Primeramente, damos la definición de estos operadores.

Definición: Un álgebra de von Neumann $A$ en un espacio de Hilbert $\mathcal{H}$ es una subálgebra de $\mathcal{B}(\mathcal{H})$ tal que $A=A^{\prime \prime}$.

Un álgebra $A$ de von Nemmann tal que $T^{*} \in A$ siempre que $T \in A$ se llama $*$-álgebra.

En particular $\mathcal{B}(\mathcal{H})$ os un álgebra de von Nemann.

Definición: Una derivación $\delta$ de un álgebra de von Neumann $A$ es un operador lineal de una ${ }^{*}$-subálgebra $D(\delta)$, el dominio de $\delta$, en $U$ con las siguientes propiedades

(i) $\delta(A)^{*}=\delta\left(A^{*}\right), A \in D(\delta)$,

(ii) $\delta(A B)=\delta(A) B+A \delta(B), A, B \in D(\delta)$.

Teorema: Sea $\mathcal{A}$ un algebra de von Neuman y $\delta$ una derivación de $\mathcal{A}$. Entonces existe un $T_{0} \in \mathcal{A}$ tal que $\left\|T_{0}\right\| \leq \delta$ y $\delta(T)=\left[T_{0}, T\right]$ para todo $T \in \mathcal{A}$. 
Demostración: $1^{\circ}$ Sea $\mathcal{U}$ el grupo de los operadores mitarios en $\mathcal{A}$. Parrat $U \in \mathcal{U}$, definimos la aplicación $A_{U}: \mathcal{A} \rightarrow \mathcal{A}$ por

$$
A_{U}(T)=(U T+\delta(U)) U^{-1} .
$$

Si $U, V \in \mathcal{U}$, tenemos

$$
\begin{aligned}
A_{U} A_{V}(T) & =\left[U(V T+\delta(V)) V^{-1}+\delta(U)\right] U^{-1} \\
& =U V T V^{-1} U^{-1}+U \delta(V) V^{-1} U^{-1}+\delta(U) U^{-1} \\
& =(U V T+\delta(U V)) V^{-1} U^{-1}
\end{aligned}
$$

entonces

$$
A_{U} A_{V}=A_{U V}
$$

Sea $\mathcal{E}$ el conjunto de $\mathcal{K} \subset \mathcal{A}$ que poseen las siguientes propiedarles:

(i) $\mathcal{K}$ es no vacío, convexo y débil compacto;

(ii) $A_{U}(\mathcal{K}) \subset \mathcal{K}$ para todo $U \in \mathcal{U}$;

(iii) cada elemento de $\mathcal{K}$ tieno norma menor igual que $\|\delta\|$

Primeramente, $\mathcal{E} \neq \emptyset$. Efectivamente, sea $\mathcal{M} \subset \mathcal{A}$ el conjunto de $\delta(U) U^{-1}$ para $U \in \mathcal{U}$. Si $U, V \in \mathcal{U}$, tenemos

$$
\begin{aligned}
A_{V}\left(\delta(U) U^{-1}\right) & =\left(V \delta(U) U^{-1}+\delta(V)\right) V^{-1} \\
& =\delta(V U) U^{-1} V^{-1}
\end{aligned}
$$

Así, $A_{V}(\mathcal{M}) \subset \mathcal{M}$. Consecuentemente, la envolvente convexo debilmente cerrada de $\mathcal{M}$ es un elemento de $\mathcal{E}$. Si $\left(\mathcal{K}_{i}\right)$ es una familia totalmente ordenarla de conjuntos $\mathcal{E}$, tenemos $\cap \mathcal{K}_{i} \in \mathcal{E}$. Por el lema de Zom, existe en $\mathcal{E}$ un elemento minimal $\mathcal{K}_{0}$.

Supóngase que se ha probado que $\mathcal{K}_{0}$ se reduce a un solo punto $T_{\mathbf{0}}$, en otras palabras $\mathcal{K}_{0}-\mathcal{K}_{0}=\{0\}$. Tenemos entonces, para cada $U \in \mathcal{U}$,

$$
T_{0}=A_{U}\left(T_{0}\right)=U T_{0} U^{-1}+\delta(U) U^{-1}
$$

por tanto $\delta(U)=\left[T_{0}, U\right]$ y por linealidad $\delta(T)=\left[T_{0}, U\right]$ para cada $T \in \mathcal{A}$.

Nótese que $\mathcal{K}_{0}-\mathcal{K}_{0}$ es convexo y débil-compacto, y que

$$
U\left(\mathcal{K}_{0}-\mathcal{K}_{0}\right) U^{-1}=\mathcal{K}_{0}-\mathcal{K}_{0}
$$

para carla $U \in \mathcal{U}$, daclo que

$$
U(S-T) U^{-1}=A_{U}(S)-A_{U}(T)
$$


para $S, T \in \mathcal{K}_{0}$.

$2^{\circ}$ Si $\mathcal{A}$ es el producto de álgebras de von Neumann $\mathcal{A}_{1}, \mathcal{A}_{2}$, tenemos $\delta\left(\mathcal{A}_{1}\right) \subset \mathcal{A}_{1}$ y $\delta\left(\mathcal{A}_{2}\right) \subset \mathcal{A}_{2}{ }^{18}$, Podemos así, suponer que $\mathcal{A}$ es semi-finita o puramente infinita ${ }^{19}$. Si $\mathcal{A}$ es semi-finita, el conjunto de proyecciones $E$ de $\mathcal{A}$ tales que $\mathcal{A}_{E}$ es finita es un filtro creciente con supremo $I^{20}$. Por lo tanto, es suficiente demostrar sólo el caso cuando $\mathcal{A}$ es finita y $\sigma$-finita ${ }^{21}$.

$3^{\circ}$ Supóngase que $\mathcal{A}$ es finita y $\sigma$-finita, y $\operatorname{sean} S, T \in \mathcal{K}_{0}$. Entonces, existe una traza finita, normal confiable ${ }^{22}$ en $\mathcal{A}$; Esta traza define una norma $\|\cdot\|_{2}$ pre-Hilbert en $\mathcal{A}$. Sea $\alpha=\sup _{R \in \mathcal{K}_{0}}\|R\|_{2}<+\infty$. Sea $H \subset \mathcal{K}_{0}$ el conjunto de $A_{U}\left(\frac{1}{2}(S+T)\right)$ para $U \in \mathcal{U}$; sea $H^{\prime} \subset \mathcal{K}_{0}$ la componente convexa cerrada en la topología débil de $H$; por (i), $H$ es invariante bajo los $A_{U}$, entonces también lo es $H^{\prime}$, entonces $H^{\prime}=\mathcal{K}_{0}$ por la minimilidad de $\mathcal{K}_{0}$. Para cada $\varepsilon>0$, existe $m R \in \mathcal{K}_{0}$ tal que $\alpha-\varepsilon<\|R\|_{2}$; como la norma \|\|$_{2}$ es semicontinua por abajo en la topología débil ${ }^{23}$, existe un operador $U \in \mathcal{U}$ tal (juc)

$$
\begin{aligned}
\alpha-\varepsilon & <\left\|A_{U}\left(\frac{1}{2}(S+T)\right)\right\|_{2} \\
& =\left\|\frac{1}{2}\left(A_{U}(S)+A_{U}(T)\right)\right\|_{2} .
\end{aligned}
$$

Dado que $\left\|A_{U}(S)\right\|_{2} \leq \alpha$ y $\left\|A_{U}(T)\right\|_{2} \leq \alpha$ y $\|\cdot\|_{2}$ es una norma pre-Hibert, existe un $U$ tal que

$$
\begin{aligned}
\left\|A_{U}(S)-A_{U}(T)\right\|_{2} & =\left\|U(S-T) U^{-1}\right\|_{2} \\
& =\|S-T\|_{2} .
\end{aligned}
$$

Por tanto $S=T$.

$4^{\circ}$ Supóngase que $\mathcal{A}$ es puramente infinita y $\sigma$-finita, y sean $S, T \in \mathcal{K}_{0}$. Sea $f$ una forma lineal débil continua en $\mathcal{A}$. Sea $\alpha=\sup _{R \in \mathcal{K}_{0}}|f(R)|<\infty$. Argumentando exactamente como en $3^{0}$ : para cada $\varepsilon>0$, existe $U$ tal que

$$
\alpha-\varepsilon \leq\left|\frac{1}{2}\left(f\left(A_{U}(S)\right)+f\left(A_{U}(T)\right)\right)\right| ;
$$

\footnotetext{
${ }^{18}$ Dixmier $[D]$, Von Nenman Algebras, Lema 2, Parte III, capítulo 9.

"19Dixmicr, op.cit. capútulo 6

${ }^{20}$ Dixmior, op.cit. cap.2, prop. 5 y coro. 1 de la prop. 7

${ }^{21}$ Dixmicr, op.cit. (aip.9) lemits 5 y 6

${ }^{22}$ Una forma lineal $\Phi$ se llama confiable si $\Phi(T)=0$ y $T^{\prime} \in \mathcal{A}^{+}$implica que $T=0$.

${ }^{23}$ Dixmier, op.cit. parte I capítulo 6, corolario de la proposición 2
} 
como $f\left(A_{U}(S)\right) \leq \alpha$ y $f\left(A_{U}(T)\right) \leq \alpha$, existe $U \in \mathcal{U}$ tal que

$$
\left|f\left(A_{U}(S)-A_{U}(T)\right)\right|=\left|f\left(U(S-T) U^{-1}\right)\right|
$$

es suficientemente pequeño.

Si $\mathcal{K}_{0}-\mathcal{K}_{0} \neq 0$, podemos escoger $S, T \in \mathcal{K}_{0}$ es un elemento no cero del centro $^{24}$ de $\mathcal{A}$; entonces

$$
U(S-T) U^{-1}=S-T
$$

para todo $U \in \mathcal{U}$, y por lo demostrado en este párrafo tenemos que $f(S-T)=$ 0. Por tanto $S-T=0$, lo cual es una contradicción.

\footnotetext{
${ }^{24}$ Dixnier, op.cit. parte III, cap 8 ,coro.6 del teo.1
} 


\section{Apendice 4}

\section{Teorema de existencia de la medida de Haar.}

Sea $X$ un grupo topológico, Hausdorff, localmente compacto. Una medida de Iraar sobre $X$ es ma merlida de Borel $\mu$, no identicamente cero que es invariante por la izquierda y por la derecha:

$$
\begin{aligned}
& \mu(x S)=\mu(S) \\
& \mu(S x)=\mu(S),
\end{aligned}
$$

para todo boreliano $S \subset X$ y para todo punto $x \in X$.

Lema: Sea $G$, un abierto de Borel no vacio, fijo. Para un compacto no vacio $C$, considérese el conjunto de números naturales $n$, que tienen la propiedad de que existe una serie de puntos $x_{1}, \ldots, x_{n}$ tales que $C \subseteq \cup_{1}^{n} x_{i} G$. Dado que $C$ es compacto, este conjunto de enteros es no vacío. El simbolo $C: G$, designa el natural más pequeño de este conjunto con esta propiedad. Si $C=\emptyset$ diremos por definición que $C: G=\emptyset: G=0$.

Podemos definir una función entera sobre $\mathcal{C}=\{C: C$ es compacto en $X\}$ en lérminos de un parámetro $G \neq \emptyset$ :

$$
C \rightarrow C: G
$$

Entonces $C \rightarrow C: G$ tiene las siguientes propiedades:

(i) Invarianza por la izquierda.

(ii) $C \rightarrow C: G$ es real (de hecho natural) y monótona.

(iii) Subaditividad finita. 
(iv) Aditividad débil èn sentido finito.

Demostración:(i) Invarianza por la izquierda:

$$
(y C): G=c: G
$$

para todo $C \in \mathcal{C}, y \in X$.

Ya que $C \subseteq \cup_{i=1}^{n} x_{i} G$ si y sólo si $C \subseteq \cup_{i=1}^{n}\left(y x_{i}\right) G$

(ii) $C \rightarrow C: G$ es real (de hecho natural) y monótona:

$$
C_{1} \supseteq C_{2} \text { implica } C_{1}: G \geq C_{2}: G
$$

Esto se prueba, haciendo notar que si $C_{1} \supseteq C_{2} \neq \emptyset$ y $x_{1}, \ldots x_{n}$ es una serie tal que

$$
C_{1} \subset \cup_{i=1}^{n} x_{i} G
$$

entonces,

$$
C_{2} \subset \cup_{i=1}^{n} x_{i} G
$$

de modo que $n \geq C_{2}: G$.

(iii) Subaditividad finita:

$$
\left(C_{1} \cup C_{2}\right): G \leq C_{1}: G+C_{2}: G
$$

puesto que si $\cup_{i=1}^{n_{1}} x_{i} C_{,} \cup_{i=1}^{n_{2}} x_{i} G$, cubren respectivamente a $C_{1}, C_{2}$ donde $n_{1}=$ $C_{1}: G, n_{2}=C_{2}: C$ entonces

$$
\left(\cup_{i=1}^{n_{1}} x_{i} G\right) \cup\left(\cup_{i=1}^{n_{2}} x_{i} G\right)
$$

cubre a $C_{1} \cup C_{2}$ y por lo tanto

$$
\left(C_{1} \cup C_{2}\right): G \leq n_{1}+n_{2}
$$

(iv) Aditividad débil en sentido finito: Si $C_{1} G^{-1} \cap C_{2} G^{-1}=\emptyset$, entonces

$$
\left(C_{1} \cup C_{2}\right): G=C_{1}: G+C_{2}: G .
$$

En efecto, supongamos que $C_{1} \cap x G \neq \emptyset, x \in C_{2} G^{-1}$ entonces existen $c_{1} \in$ $C_{1}, g \in G$ tales que $c_{1}=x g$, así $x=c_{1} g^{-1} \in C_{1} G^{-1}$. Similarmente, si

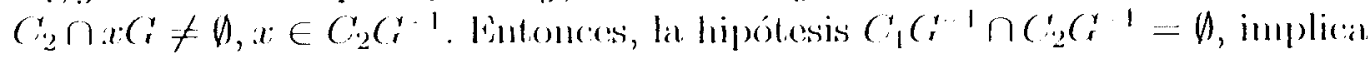
que para todo $x \in X$, al menos una de las intersecciones $C_{1} \cap x G, C_{2} \cap x G$ 
es vacía. Supongamos que $C_{1}, C_{2}$ son no vacíos y $C_{1} G^{-1} \cap C_{2} G^{-1}=\emptyset$. Sea $\left(C_{1} \cup C_{2}\right): G=n$, entonces existen $x_{1}, \ldots, x_{n}$ tales que

$$
C_{1} \cup C_{2} \subseteq \cup^{n} x_{i} G
$$

Se sigue de la hipótesis, que existe un $k \in \mathrm{N}, 1 \leq k \leq n$, tal que después de alguna permutación de los índices

$$
C_{1} \subseteq \cup_{i=1}^{k} x_{i} G, C_{2} \subseteq \cup_{i=k}^{n} x_{i} G
$$

entonces

$$
\left(C_{1} \cup C_{2}\right): G=n=k+(n-k) \geq C_{1}: G+C_{2}: G
$$

Sea $A \in \mathcal{C}$ con interior no vacío. Para $C \in \mathcal{C}$, definimos $C: A=0$ si $C=\emptyset$, y si $C \neq \emptyset, C: A$ es el mínimo natural $n$ para el cual existe ma serie $x_{1}, \ldots, x_{2}$ tial qus:

$$
C \subseteq \cup_{1}^{n} x_{i} A
$$

Sean $A$ un compacto con $\operatorname{int}(A) \neq \emptyset, G \neq \emptyset, C$ un compacto:

$$
C: G \leq(C: A)(A: G)
$$

Efectivamente, Supongamos que $C \neq \emptyset$. Si

$$
C_{1} \subseteq \cup_{i=1}^{m} x_{i} A, A \subseteq \cup_{i=1}^{k} y_{j} G
$$

entonces

$$
\begin{aligned}
C & \subseteq \cup_{i=1}^{m} x_{i}\left(\cup_{j=1}^{n} y_{j} G\right) \\
& =\cup_{i=1}^{m} \cup_{j=1}^{n}\left(x_{i} y_{j}\right) G,
\end{aligned}
$$

de manera que

$$
m n \geq C: G
$$

Consideremos $C$ : $G$ como una función de dos variables:

$$
(C, C:) \rightarrow C: C
$$

$C \in C, \theta \neq C, C$ abierto de $X$. 
Queremos una forma modificada de esta función, que sea acotada, considerada como una función de $G$, para $C$ fijo. Sea $A$ un compacto con interior no nulo. Definimos para $G \neq \emptyset$, la función $\lambda_{G}$ sobre $C$ por la fómula

$$
\lambda_{G}(C)=\frac{C: G}{A: G}
$$

$\operatorname{por}(A 4.1)$

$$
0 \leq \lambda_{G}(C) \leq C: A
$$

Así, para $C$ fijo (y $A$ fijo), $C: A$ es una cota superior de $\lambda_{C}(C)$. Supongantos $A$ fijo para todas las funciones de la forma $\lambda_{G}(G)$, entonces las variables son $G, C$. Para $G$ fijo $C \rightarrow \lambda_{G}(C), C \rightarrow C: A$, son las mismas funciones salvo un factor positivo, entonces $C \rightarrow \lambda_{G}(C)$ posee las mismas propiedades (i), (ii), (iii), (iv) establecidas para $C \rightarrow C: A$.

Teorema: Todo grupo topológico Hausdorff localmente compacto posee una única medida de Haar regular.

Demostración: Sea $X$ un gmpo topológico, Ilausslorff y localmente compacto. Sea $A \subset X$ un compacto con interior no vacío. A cada $C \in \mathcal{C}$ le asociamos un intervalo cerrado y acotado (por tanto compacto) $[0, C: A]$. El espacio producto, es entonces compacto y llausdorfl:

$$
\Phi=\Pi_{C \in \mathcal{C}}[0, C: A]
$$

Es conveniente identificar un elemento $\left(x_{i}\right)$ de $\Phi$ con la función real $\phi$ definicla en $C$ :

$$
\phi(C)=x_{i} \in[0, C: A]
$$

La proyección del espacio producto sobre el espacio factor $[0, C: A]$, para $C$ dado, es

$$
\phi \rightarrow \phi(C),(\phi \in \Phi)
$$

así, esta función, de parámetro $C$, es real y continua (por la definición, la base de la topología producto está dara por estas proyecciones).

Sea $\mathcal{W}$ la hase de $\mathcal{V}_{e}$ formada de las vecindades abiertas de Borel de la irlentidad e, del grupo $X$. Para $C \in \mathcal{C}, U \in \mathcal{W}$,

$$
0 \leq \lambda_{U}(C) \leq C: A
$$


Entonces, cada $U \in \mathcal{W}$ determina el subconjunto no nulo de $\Phi$ :

$$
\Omega(U)=\left\{\lambda_{V}: U \supseteq V \in \mathcal{W}\right\}
$$

Sean $U_{1}, \ldots, U_{n} \in \mathcal{W}$. Entonces $\cap_{1}^{n} U_{i} \in \mathcal{W}$, y $\cap_{1}^{n} U_{i} \subseteq U_{j}(j=1, \ldots, n)$ así

$$
\Omega\left(\cap_{1}^{n} U_{i}\right) \subseteq \cap_{1}^{n} \Omega\left(U_{i}\right) .
$$

Ya que para $U \in \mathcal{W}, \Omega(U)$ es no vacío $\left(\lambda_{U} \in \Omega(U)\right)$ y

$$
\cap_{1}^{n} \Omega\left(U_{i}\right) \supseteq \Omega\left(\cap_{1}^{n} U_{i}\right) \neq \emptyset
$$

Esto establece la propiedad de intersecciones finitas para la familia de subconjuntos de $\Phi:\{\Omega(U)\}_{U \in \mathcal{W}}$. Dado que $\Phi$ es compacto,

$$
\cap_{U \in \mathcal{W}} \overline{\Omega(U)} \neq \emptyset
$$

Así, existe $\lambda \in \cap_{U \in \mathcal{W}} \overline{\Omega(U)}$. Demostraremos etapa por etapa que $\lambda$ es una medida distinta de cero, que es invariante por la izcunierda y siendo un elemento de $\Phi$, es una función real, no negativa sobre $\mathcal{C}$.

(i) $\lambda$ es monótona. En efecto, sean $C_{1} \subseteq C_{2}$. Ya que $\phi \rightarrow \phi\left(C_{2}\right)-\phi\left(C_{1}\right)$ es continua,.

$$
\Delta=\left\{\phi \in \Phi: \phi\left(C_{1}\right) \leq \phi\left(C_{2}\right)\right\}
$$

es cerrado. para $U \in \mathcal{W}, \lambda_{U}$ es una función monótona sobre $\mathcal{C}$, entonces $\lambda_{U} \in \Delta$. Dado que $U$ es un elemento cualquiera de $\mathcal{W}, \Omega(U) \subseteq \Delta$, entonces

$$
\lambda \in \overline{\Omega(U)} \subseteq \Delta
$$

es decir,

$$
\lambda\left(C_{1}\right) \leq \lambda\left(C_{2}\right) .
$$

Las demás propiedades de $\lambda$ serán establecidas por el mismo argumento de continuidad.

(ii) Para dos compactos dados $C_{1}, C_{2}$, el conjunto

$$
\Delta=\left\{\phi \in \Phi: \phi\left(C_{1} \cup C_{2}\right) \leq \phi\left(C_{1}\right)+\phi\left(C_{2}\right)\right\}
$$

es cerrado (dado que $\phi \rightarrow \phi\left(C_{1} \cup C_{2}\right)-\phi\left(C_{1}\right)-\phi\left(C_{2}\right)$ es continua). Para $U \in \mathcal{W}, \lambda_{U}$ es subaditiva en sentido finito, entonces $\lambda_{U} \in \Delta$. Así, $\Omega(U) \subseteq$ $\Delta, \lambda \in \Omega(U)$, es decir,

$$
\lambda\left(C_{1} \cup C_{2}\right) \leq \lambda\left(C_{1}\right)+\lambda\left(C_{2}\right)
$$


(iii) Supongamos que $C_{1} \cap C_{2}=\emptyset$. Existen vecindades $W_{1}, W_{2}$ de $C_{1}, C_{2}$ respectivamente, tales que $W_{1} \cap W_{2}=\emptyset$. Existe una vecindad $V_{1}$ de $e$ tal que $C_{1} V_{1} \subseteq W_{1}$, entonces existe una vecindad abierta de Borel $U_{1}$ de $e$ tal que $U_{1}^{-1} \subseteq V_{1}$, de suerte que

$$
C_{1} U_{1}^{-1} \subseteq C_{1} V_{1} \subseteq W_{1}
$$

De la misma manera, existe una vecindad abierta de Borel $U_{2}$ de $e$ tal que

$$
C_{2} U_{2}^{-1} \subseteq W_{2}
$$

$U=U_{1} \cap U_{2}$ es una vecindad abierta de Borel de $e$, tal que $C_{1} U^{-1} \subseteq W_{1}$, $C_{2} U^{-1} \subseteq W_{2}$, así $C_{1} U^{-1} \cap C_{2} U^{-1}=\emptyset$.

Para la aditividad clébil en sentido finito,

$$
\lambda_{U}\left(C_{1}+C_{2}\right)=\lambda_{U}\left(C_{1}\right)+\lambda_{U}\left(C_{2}\right) .
$$

El conjunto

$$
\Delta=\left\{\phi \in \Phi: \phi\left(C_{1}+C_{2}\right)=\phi\left(C_{1}\right)+\phi\left(C_{2}\right)\right\}
$$

es cerrado, y hemos mostrado que existe $U \in \mathcal{W}$ tal que $\Omega(U) \subseteq \Delta$, así $\lambda \in \overline{\Omega(U)} \subseteq \Delta$, es decir

$$
\lambda\left(C_{1}+C_{2}\right)=\lambda\left(C_{1}\right)+\lambda\left(C_{2}\right) .
$$

(iv) $\Delta=\{\phi \in \Phi: \phi(A)=1\}$ es cerrado. Para $U \in \mathcal{W}$,

$$
\lambda_{U}(A)=\frac{A: U}{A: U}=1,
$$

entonces $\Omega(U) \subseteq \Delta, \lambda \in \overline{\Omega(U)} \subseteq \Delta$, es decir,

$$
\lambda(A)=1
$$

en particular, $\lambda$ no es identicamente cero.

(v) Dado $C$ y $x \in X$, el conjunto

$$
\Delta=\{\phi \in \Phi: \phi(x C)=\phi(C)\}
$$

es cerrado. Para $U \in \mathcal{W}, \lambda_{U}$ es invariante por la derecha, entonces $\Omega(U) \subseteq$ $\Delta, \lambda \in \overline{\Omega(\bar{U})} \subseteq \Delta$, es decir,

$$
\lambda(x C)=\lambda(C)
$$


$\lambda$ induce una medida de Borel $\mu$ regular. Dado que

$$
\lambda(C) \leq \mu(C)
$$

.$\mu$ no es identicamente cero. Por el teorema de invarianza, $\mu$ es invariante por la izquierda, es decir, $\mu$ es una medida regular de Haar.

Finalmente, establecemos sin demostración el siguiente teorema

Teorema: Sea $G$ un grupo topológico localmente compacto. Existe un funcional I en $\mathcal{C}_{00}^{+}$tal que:

(i) $I(f)$ es real y positiva para $f \neq 0$.

(ii) $I(f+g)=I(f)+I(g)$ para toda $f, g \in \mathcal{C}_{00 \text {. }}^{+}$

(iii) $I(\alpha f)=\alpha I(f)$ para $\alpha \geq 0$ y $f \in \mathcal{C}_{00}^{+}$.

(iv) $I\left(f_{U}\right)=I(f)$ para $U \in G$ y $f \in \mathcal{C}_{00}^{+}$, donde $f_{U}(X)=f(X U)$ para $U, X \in G$; similarmente $I\left({ }_{u} f\right)=I(f)$ con ${ }_{U} f(X)=f(U X)$.

Donde $\mathcal{C}_{00}$ y $\mathcal{C}_{00}^{+}$son el conjunto de funciones de soporte compacto y el conjunto de funciones no negativas de soporte compacto en $G$ respectivamente. Al funcional I se le conoce como integral de Haar en $\mathcal{C}_{00}^{+}$. 


\section{Apendice 5}

\section{Productos tensoriales, sumas directas y trazas de operadores.}

\section{Productos tensoriales de espacios de Hilbert.}

Valiéndonos del teorema de construccion de Gelfand-Naimark-Segal del apéndice 1, podemos introducir el producto tensorial de espacios de Hilbert de la siguiente manera:

Definición: Sean $\mathcal{H}_{i}, 1 \leq i \leq n$, espacios de Hilbert y sea $\mathcal{H}^{\prime}=\mathcal{H}_{1} \times \cdots \times \mathcal{H}_{n}$ entonces la función

$$
K(\underline{u}, \underline{v})=\prod_{i-1}^{n}\left\langle u_{i}, v_{i}\right\rangle, \underline{u}=\left(u_{1}, \ldots, u_{n}\right), \underline{v}=\left(v_{1}, \ldots v_{n}\right)
$$

donde $u_{i}, v_{i} \in \mathcal{H}_{i}$ es un kernel en $\mathcal{H}^{\prime}$. Considérese el par de Gelfand $(\mathcal{H}, \pi)$ asociarlo a $\left(\mathcal{H}^{\prime}, K\right)$ entonces $\mathcal{H}$ es llamado producto tensorial de $\mathcal{H}_{i}, i=$ $1, \ldots, n$. Escribimos

$$
\begin{aligned}
\mathcal{H} & =\mathcal{H}_{1} \otimes \mathcal{H}_{2} \otimes \cdots \otimes \mathcal{H}_{n}=\bigotimes_{i=1}^{n} \mathcal{H}_{i} \\
\pi(\underline{u}) & =u_{1} \otimes \cdots \otimes u_{n}=\bigotimes_{i=1}^{n} u_{i}
\end{aligned}
$$

y llamamos a $\pi(\underline{u})$ el producto tensorial de los vectores $u_{i}$.

A continuación enunciamos algunas propiedades: 
Proposición: Sean $\mathcal{H}_{i}, 1 \leq i \leq n$, espacios de Hilbert y sea $T_{i}$ un operador acotado en $\mathcal{H}_{i}$ para cada $i$. Entonces, existe un operador $T$ único en $\mathcal{H}=$ $\otimes_{i=1}^{n} \mathcal{H}_{i}$ que satisface:

$$
T \otimes_{i=1}^{n} u_{i}=\otimes_{i=1}^{n} T_{i} u_{i} \text { para todo } u_{i} \in \mathcal{H}_{i}, 1 \leq i \leq n .
$$

además $\|T\|=\Pi_{i}\left\|T_{i}\right\|$.

Proposición: Sea $\mathcal{H}_{i}, 1 \leq i \leq n$ espacios de Hilbert y sean $S_{i}, T_{i}$ operadores acotados en $\mathcal{H}_{i}$ para cada $i$. Sean $S=\otimes_{i=1}^{n} S_{i}, T=\otimes_{i=1}^{n} T_{i}$. Entonces se cumplen las siguientes propiedades.

(i) La aplicación definida de $\mathcal{B}\left(\mathcal{H}_{1}\right) \times \cdots \times \mathcal{B}\left(\mathcal{H}_{n}\right)$ en $\mathcal{B}\left(\mathcal{H}_{1} \otimes \cdots \otimes \mathcal{H}_{n}\right)$, $\left(T_{1}, \ldots, T_{n}\right) \rightarrow T$ es multilineal

(ii) $S T=\otimes_{i=1}^{n} S_{i} T_{i}, T^{*}=\otimes_{i=1}^{n} T^{*}$;

(iii) Si cada $T_{i}$ tiene una inverso acotado entonces $T$ tiene inverso acotado $y T^{-1}=\otimes_{i=1}^{n} T_{i}^{-1}$;

(iv) $T$ es autoadjunto, unitario, normal, o proyección cuando cada uno de: los $T_{i}$ es autoadjunto, unitario, normal o proyección;

(v) $T$ es positivo si cada $T_{i}$ es positivo;

(vi) Si $T_{i}=\left|u_{i}\right\rangle\left\langle v_{i}\right|$ donde $u_{i}, v_{i} \in \mathcal{H}_{i}$ para cada $i$ entonces

$$
T=\left|u_{1} \otimes \cdots \otimes u_{n}\right\rangle\left\langle v_{1} \otimes \cdots \otimes v_{n}\right| .
$$

\section{Sumas directas de operadores}

Definición: Sea $T_{j}$ un operador autoadjunto en $\mathcal{H}_{j}, j=1,2, \ldots$ En el espacio de Hilbert $\mathcal{H}=\oplus \mathcal{H}_{j}$ definimos

$$
\begin{gathered}
D(T)=\left\{u \mid u=\underset{j}{\oplus} u_{j}, u_{j} \in D\left(T_{j}\right), \sum_{j}\left\|T_{j} u_{j}\right\|<\infty\right\} \subset \mathcal{H}, \\
T \underset{j}{\oplus} u_{j}:=\underset{j}{\oplus} T_{j} u_{j} .
\end{gathered}
$$

Proposición: Sea $A_{n} \in \mathcal{B}\left(\mathcal{H}_{n}\right), n=1,2, \ldots$, donde $\left\{\mathcal{H}_{n}\right\}$ es una sucesión de espacios de Hilbert. Suponga que sup $\left\|A_{u}\right\|<\infty$. Fntonces existe un uinico operador $A=\oplus_{n} A_{n}$ en $\mathcal{H}=\oplus_{n} \mathcal{H}_{n}$ que satisface

$$
A \oplus_{n} u_{n}=\oplus_{n} A_{n} u_{n}
$$


además

$$
\|A\|=\sup _{n}\left\|A_{n}\right\| .
$$

Si $\left\{A_{n}\right\},\left\{B_{n}\right\}$ son dos sucesiones de operadores lales que $A_{n}, B_{n} \in \mathcal{B}\left(\mathcal{H}_{n}\right)$ para cada $n$ y $\sup _{n}\left(\left\|A_{n}\right\|+\left\|B_{n}\right\|\right)<\infty$ entonces sus sumas directas $A=$ $\oplus_{n} A_{n}$ y $B=\oplus_{n} B_{n}$ satisfacen lo siguiente:

(i) $A+B=\oplus_{n}\left(A_{n}+B_{n}\right), A B=\oplus_{n} A_{n} B_{n}, A^{*}=\oplus_{n} A_{n}^{*}$;

(ii) Si cada $A_{n}$ tiene un inverso acotado y sup $\left\|A_{n}^{-1}\right\|<\infty$ entonces $A$ ticne un inverso acotado y $A^{-1}=\oplus_{n} A_{n}^{-1}$;

(iii) A es autoadjunto, normal, unitario, positivo o proyección de cuando cada $A_{n}$ liene las mismas propiedades.

(iv) $S i A$ es de la clase de traza ${ }^{25}$ entonces cada $A_{n}$ es de la clase de traza y $\|A\|_{1}=\sum_{n}\left\|A_{n}\right\|_{1}, \operatorname{tr} A=\sum_{n} \operatorname{tr} A$.

\section{Traza de operadores, principales propiedades.}

Definición: Sea $\mathcal{H}$ un espacio de Hilbert separable, $\left\{\varphi_{n}\right\}_{n=1}^{\infty}$ una base ortonormal. Entonces, para cada operador positivo $A$ definimos $\operatorname{tr} A=\sum_{n=1}^{\infty}\left\langle\varphi_{n}, A \varphi_{n}\right\rangle$. El número $\operatorname{tr} A$ es llamado traza de $A$ y es independiente de la base escogida.

Lema: La traza tiene las siguientes propiedades:

(a) $\operatorname{tr}(A+B)=\operatorname{tr} A+\operatorname{tr} B$.

(b) $\operatorname{tr}(\lambda A)=\lambda \operatorname{tr} A$ para todo $\lambda \geq 0$.

(c) $\operatorname{tr}\left(U A U^{-1}\right)=\operatorname{tr} A$ para cualquier operador unitario $U$.

(d) Si $0 \leq A \leq B$, entonces $\operatorname{tr} A \leq \operatorname{tr} B$.

Demostración:Dada mui bise ortogonal $\left\{\varphi_{n}\right\}_{n=1}^{\infty}$, definimos $\mid r_{\varphi}(A)=\sum_{n=1}^{\infty}\left\langle\varphi_{n}, A \varphi_{n}\right\rangle$. Si $\left\{\psi_{n}\right\}_{n=1}^{\infty}$ es otra base ortonormal entonces

$$
\begin{aligned}
\operatorname{tr}_{\varphi}(A) & =\sum_{n=1}^{\infty}\left\langle\varphi_{n}, A \varphi_{n}\right\rangle \\
& =\sum_{n=1}^{\infty}\left\|A^{\frac{1}{2}} \varphi_{n}\right\|^{2} \\
& =\sum_{n=1}^{\infty}\left(\sum_{m=1}^{\infty}\left|\left\langle\psi_{m}, A^{\frac{1}{2}} \varphi_{n}\right\rangle\right|^{2}\right)
\end{aligned}
$$

\footnotetext{
25 Ver la signiente sección del apéndice.
} 


$$
\begin{aligned}
& =\sum_{m=1}^{\infty}\left(\sum_{n=1}^{\infty}\left|\left\langle A \psi_{m}, \varphi_{n}\right\rangle\right|^{2}\right) \\
& =\sum_{m=1}^{\infty}\left\|A^{\frac{1}{2}} \psi_{m}\right\|^{2} \\
& =\sum_{m=1}^{\infty}\left\langle\psi_{m}, A \psi_{m}\right\rangle \\
& =t r_{\psi}(A)
\end{aligned}
$$

Dado que todos los términos son positivos, el intercambio de las sumas está permitido. Las propiedades (a), (b) se siguen facilmente de la linealidad del proclucto interior (d), también es inmediatic, para ver (c) notamos que si $\left\{\varphi_{n}\right\}$ es uma hase ortonormal, también lo es $\left\{U \varphi_{n}\right\}$. Fintonces,

$$
\begin{aligned}
\operatorname{tr}\left(U A U^{-1}\right) & =\operatorname{tr}_{(U \varphi)}\left(U A U^{-1}\right) \\
& =\operatorname{tr}_{\varphi}(A) \\
& =\operatorname{tr}(A) .
\end{aligned}
$$

Definición: Un operador $A \in \mathcal{L}(\mathcal{H})$ es llamado de la clase de traza si y sólo si $\operatorname{tr}|A|<\infty$. La familia de los operadores de la clase de traza se denota por $\mathcal{T}_{1}$.

$\mathcal{T}_{1}$ es un ${ }^{*}$-ideal bilateral en $\mathcal{L}(\mathcal{H})$, es decir: (i) $\mathcal{T}_{1}$ es un espacio vectorial; (ii) Si $A \in \mathcal{T}_{1}$ y $B \in \mathcal{L}(\mathcal{H})$; entonces $A B \in \mathcal{T}_{1}, B A \in \mathcal{T}_{1}$; (iii) Si $A \in \mathcal{T}_{1}$, entonces $A^{*} \in \mathcal{T}_{1}$.

Un elemento $T \in \mathcal{L}(\mathcal{H})$ tiene rango finito $n$ si $\operatorname{dim}(T)=n<\infty$. Se denota por $\mathcal{T}_{0}$ al conjunto de todos los operadores de rango finito. $\mathcal{T}_{0}$ es un * ideal bilateral.

Un elemento $T \in \mathcal{B}(\mathcal{H})$ se llama operador compacto si para toda sucesión $\left\{u_{n}\right\}$ de vectores unitarios en $\mathcal{H},\left\{T u_{n}\right\}$ tiene una subsucesión convergente. Denotamos por $\mathcal{T}_{\infty}$ el conjunto de operadores compactos. $\mathcal{T}_{\infty}$ es también un *-ideal bilateral.

Teorema: (Schatten) Sea $\mathcal{H}$ cualquier espacio complejo de Hilbert separable. Para cualquier operador de la clase de traza $T$ y cualquier operador acolado $X$ en $\mathcal{H}$ sen $(T, X)=t^{\prime} T X$. Entoneses se cumplen las siguitules propicadaies.

(i) Con la norma $\|T\|_{1}=t r|T|$ el conjunto $\mathcal{T}_{1}(\mathcal{H})$ de los operadores de la clase de traza en $\mathcal{H}$ es isométricamente isomorfo al dual del espacio de 
Banach $\mathcal{T}_{\infty}$ de los operadores compactos en $\mathcal{H}$ con la norma de operalores bajo la correspondencia $\left.T \rightarrow(T, \cdot)\right|_{\mathcal{T}_{\infty}(\mathcal{H})}, T \in \mathcal{T}_{1}(\mathcal{H})$;

(ii) El espacio de Banach $\mathcal{B}(\mathcal{H})$ de los operadores acotados en $\mathcal{H}$ es isométricamente isomorfo al dual de $\mathcal{T}_{1}(\mathcal{H})$ con la norma $\|\cdot\|_{1}$ bajo la correspondencia $\left.X \rightarrow(\cdot, X)\right|_{T_{1}(\mathcal{H})}, X \in \mathcal{B}(\mathcal{H})$.

Demostración: El teorema es consecuencia directa de los siguientes lemas:

Lema V.1: Sea $\lambda$ cualquier funcional acotada en el espacio de Banach $\mathcal{T}_{\infty}$. Entonces existe an $T \in \mathcal{T}_{1}$ untco, tal que $\lambda(X)=\operatorname{tr} T X$ para todo $X$. Más aún

$$
\operatorname{sil}_{\substack{X \in T_{\infty}(\mathcal{H}) \\\|X\|=1}}|\lambda(X)|=\|T\|_{1}
$$

donde: $\|T\|_{1}=$ sup $\|x\|=1 \mid$ br $T X \mid$. Conversamente, para lodo $T \in \mathcal{T}_{1}(\mathcal{H})$ la transformación $X \underset{X \in \mathcal{B}(\mathcal{H})}{\operatorname{tr} T X}$ es un funcional acotado en $\mathcal{T}_{\infty}(\mathcal{H})$.

Demostración: Para cualescuicra $u, v \in \mathcal{H},|u\rangle\langle v| \in \mathcal{T}_{\infty}$ y $B(u, v)=$ $\lambda(|u\rangle\langle v|)$ es una forma sesquilineal que satisface

$$
B(u, v)=\|\lambda\|\|u\|\|v\|
$$

Entonces existe un operador $T \in \mathcal{B}(\mathcal{H})$ tal que

$$
\begin{aligned}
\lambda(|u\rangle\langle v|) & =\langle v, T u\rangle \\
& =\operatorname{tr} T|u\rangle\langle v| \text { para todo } u, v \in \mathcal{H}
\end{aligned}
$$

Considérese la descomposición normal $T=U|T|$ y escójase una base ortonormal $\left\{u_{n}\right\}$ en $R(|T|)$ tal que $v_{j}=U u_{j}$ sea ma hase ortonormal en $R(T)$. Fntonces para cada $n$ fijo, $\sum_{j-1}^{n}\left|u_{j}\right\rangle\left\langle u_{j}\right|=X_{n}$ es moperador de ramgo finito y norma mo y

$$
\begin{aligned}
\lambda\left(X_{n}\right) & =\sum_{j=1}^{n}\left\langle v_{j}, T u_{j}\right\rangle=\sum_{j=1}^{n}\left\langle U^{*} v_{j},|T| u_{j}\right\rangle \\
& =\sum_{j=1}^{n}\left\langle u_{j}, T \mid u_{j}\right\rangle \leq\|\lambda\| .
\end{aligned}
$$


Escójase una base ortonormal de $N(|T|)=R(|T|)^{\perp}$, junte ésta con $\left\{u_{n}\right\}$ y constiruyase una base ortogonal $\left\{f_{k}\right\}$ en $\mathcal{H}$ tal que

$$
\sum_{j=1}^{n}\left\langle f_{j},|T| f_{j}\right\rangle \leq\|\lambda\|
$$

Por la proposición 9.14 del libro de Parthasarathy [P2], $T \in \mathcal{T}_{1}(\mathcal{H})$,

$$
\|T\|_{1}=\sum_{j=1}^{n}\left\langle f_{j},|T| f_{j}\right\rangle \leq\|\lambda\|
$$

y $\lambda(X)=\operatorname{tr} T X$ para todo $X$ en $\mathcal{T}_{\infty}(\mathcal{H})$. Por otra parte por la proposición 9.12 de la obra citada $\|\lambda\| \leq\|T\|_{1}$. Así, $\|\lambda\|=\|T\|_{1}$.

Lema V.2: Sea $\lambda$ cualquier funcional acotado en el espacio de Banach $\mathcal{T}_{1}(\mathcal{H})$ con $\left\|_{1}\right\|_{1}$. Entonces existe un operador único $X \in \mathcal{B}(\mathcal{H})$ tal que $\lambda(T)=\operatorname{tr} T X$ para todo $T$ en $\mathcal{T}_{1}(\mathcal{H})$ y $\|\lambda\|=\|X\|$. Recíprocamente, para todo $X$ en $\mathcal{B}(\mathcal{H})$ la transformación $T \rightarrow$ tr $T X$ es un funcional lineal acotado en $\mathcal{T}_{1}(\mathcal{H})$.

Demostración: Como en la prueba de la proposición anterior la transformación $(v, u) \rightarrow \lambda(|u\rangle\langle v|)$ es una forma sesquilineal acotada y por lo tanto existe un $X \in \mathcal{B}(\mathcal{H})$ que satisface

$$
\lambda(|u\rangle\langle v|)=\operatorname{tr} X|u\rangle\langle v| .
$$

Por la proposición 9.3 de $[P 2]$ cualquier $T \in \mathcal{T}_{1}(\mathcal{H})$ puede ser expresada como

$$
T=\sum_{j} s_{j}(T)\left|u_{j}\right\rangle\left\langle v_{j}\right|
$$

donde $\sum s_{j}(T)<\infty$ y $\left\{u_{j}\right\},\left\{v_{j}\right\}$ son conjuntos ortonormales. Entonces $\lambda(T)=\operatorname{tr} T X=\operatorname{tr} X T$. Más aún

$$
|\operatorname{tr} X T|=\left|\sum_{j} s_{j}(T)\left\langle u_{j}, X v_{j}\right\rangle\right| \leq\|X\|\|T\|_{1} .
$$

Entonces, $\|\lambda\| \leq\|X\|$. Si $T=|u\rangle\langle v|$ donde $\|u\|=\|v\|=1$ entonces $|\operatorname{tr} X T|=|\langle v, X u\rangle| \mathrm{y}\|T\|_{1}=1$. Entonces

$$
\sup _{\|T\|_{1}=1}|\operatorname{tr} X T|=\|X\|
$$


$\mathrm{y}\|\lambda\|=\|X\|$.

LemaV.3: Sea $T$ que pertence a $\mathcal{B}(\mathcal{H})$, entonces $T$ es combinación lineal de operadores unitarios.

Demostración: Cada $T \in \mathcal{B}(\mathcal{H})$ puede escribirse de manera uníca de la forma $T=T_{1}+i T_{2}$, donde $T_{1}, T_{2}$ son hermitianos. En efecto,

$$
T_{1}=\frac{1}{2}\left(T+T^{*}\right) \text { y } T_{2}=\frac{i}{2}\left(T^{*}-T\right)
$$

Considerando este hecho ahora es suficiente considerar el caso cuando $T$ es hemitiano tal que $\|T\| \leq 1$. En este caso $I-T^{2} \geq 0$. Escribiendo

$$
U=T+i\left(I-T^{2}\right)^{\frac{1}{2}}
$$

tenemos que $U \in \mathcal{U}(\mathcal{H})$ y

$$
\begin{aligned}
U U^{*} & =U^{*} U=\left(T+i\left(I-T^{2}\right)^{\frac{1}{2}}\right)\left(T-i\left(I-T^{2}\right)^{\frac{1}{2}}\right) \\
& =T^{2}+I-T^{2}=I
\end{aligned}
$$

así que $U$ es unitario. Más aún

$$
T=\frac{1}{2}\left(U+U^{*}\right)
$$




\section{Bibliografía}

[A] Alicki y Lendi, Quantum Dynamical Semigroups, Lectures Notes on Mathematical Physics.

[Br] Bratelli, PET, Jørgensen, A, Kishimoto, and D.W. Robinson, $A C^{*}$ algebraic Schoenberg theorem, Ann. Inst. Fourier (Grenoble)34, 155-187, 1984 .

[Ch1]Chebotarev A.M. and Fagnola F., On a remarkable quantum dynamical semigroup, Lect. Notes Math.1613,1-16,1995.

[Ch2] Chebotarev, A.M., The theory of conservative dynamical semigroups and its applications, preprint MIEM, 1990.

[Ch:3] Cholotiarev, A.M. Sufficicnl condilions of the consenalism of a minimal dynamical semigroup. Math Notes, vol 52. N.4, pp112-127. 1992.

$\left[C h_{-}-F\right]$ Chebotarev, A.M. and Fagnola, F. Sufficient conditions for conservativity of quantum dynamical semigroups. J.Funct: Anal. 118 (1993), pp.131153.

[Chr-E] Christiansen E and Evans D.E. Cohomology of operator algebras and quantum dynamical semigroups, J. Funct.Anal. 118,131-153,1993.

[Da1] Davis, E.B. Quantum theory of open systems, Academic Press, 1976.

[Da2] E.B: Davis Generators of dynamical Semigroups, J. Funct. Analysis 34, 421-432, 1979 .

[D] Dixmier, Von Neumann Algebras, North Holland Mathematical Library 1981.

$[E-L]$ Evans D.E. and Lewis J.T, Dilations of irreversible evolutions in algebraic quantum theory. Comm. Dublin Inst. Ach. Stud. Ser. A 24,1997. [E1] Encry M., On Azema's martingales,Sem. Prob.XXIII, Lect. Notes Math. 1372, 67-87, 1989.

[E2] Emery M., Sur les martingales d'Azema, Sem. Prob. XXIV,442447,1990 .

[F-M] Fagnola, F. y Monte, R. Quantum extensions of semigroups generated 
by Bessel Processes, Math Notes Russian Acal. Sci. Vol 60, 389-401.

$[G]$ García J.C., Divergence criteria for random series related to the Azéma-

Emery martingale process, Math. Notes Russian Acad. Sci. 57, 361-368, 1995.

[G-Q] García, J.C.and Quezada, R., On strong perturbations of covariant generalors of quantum dynamical semigroups; en memorias del IV Simposio de Probabilidad y procesos estocásticos, Aport. Mat. 12, 91-105, 1996.

[G-K-S] Gorini, V., Kossakowski, A., Sudarshan, E.C.G., Completely positive dynamical semigroups of n-level systems, J.Math.Phys.17,821-825,1976.

[II] A.S. Holevo, On conservativity of covariant dynamical semigroups, Rep. Math. Phys. ,33, pp 95-100, 1993.

[H-P] Hudson R.L. Parthasarathy K.R. Quantum Ito's formula and estochastic evolution Comm. Math Phys. 93,301-323, 1984.

[P2] Parthasarathy, K.R. An introduction to quantum stochastic calculus, Birkhäuser Verlag, 1992.

[P1] Parthasarathy, K.R. Azéma Martingales and quantum estochastic Calculus, Proc. R.C. Bose Symposium, 551-569

$[S-R]$ Simon B.and Reed M. Methods of Modern Mathematical Physics, Academic Press 1980.

[S] Sunder, V.S., An invitation to Von Neumann Algebras. Academic press. $[K a]$ Kato, T. Perturbation Theory for lineal operators, Springer Verlag 1980. $[K]$ Kraus, K. General estate changes in Quantum theory, Anm.Phys. 64,311335.

[L] Lindblad 1976, On the generators of quantum dinamical semigroups, Comm. Math. Phys.48, 119-130, 1976

$[M]$ Meyer, P.A., Quantum Probability for probabilists, Lecture Notes in Math 1338, 1993.

$[W]$ Weidnann, J. Linear Operators in Ililbert Spaces, Springer Verlag, 1980. [Y] Yosida, Kôsaku, Functional Analysis, Springer-Verlag 1978. 